\title{
Wall effects on pressure fluctuations in turbulent channel flow
}

\author{
G. A. GER O L YMOS, D. SENECHA L, I. VALLET \\ Université Pierre-et-Marie-Curie (UPMC), 4 place Jussieu, 75005 Paris, France
}

(Received 20 November 2018)

The purpose of the present paper is to study the influence of wall-echo on pressure fluctuations $p^{\prime}$, and on statistical correlations containing $p^{\prime}$, viz redistribution $\phi_{i j}$, pressure diffusion $d_{i j}^{(p)}$, and velocity/pressure-gradient $\Pi_{i j}$. We extend the usual analysis of turbulent correlations containing pressure fluctuations in wall-bounded DNS computations [Kim J.: J. Fluid Mech. 205 (1989) 421-451], separating $p^{\prime}$ not only into rapid $p_{(\mathrm{r})}^{\prime}$ and slow $p_{(\mathrm{s})}^{\prime}$ parts [Chou P.Y.: Quart. Appl. Math. 3 (1945) 38-54], but further into volume $\left(p_{(\mathrm{r} ; \mathfrak{V})}^{\prime}\right.$ and $\left.p_{(\mathrm{s} ; \mathfrak{T})}^{\prime}\right)$ and surface (wall-echo; $p_{(\mathrm{r} ; w)}^{\prime}$ and $\left.p_{(\mathrm{s} ; w)}^{\prime}\right)$ terms. An algorithm, based on a Green's function approach, is developed to compute the above splittings for various correlations containing pressure fluctuations (redistribution, pressure diffusion, velocity/pressure-gradient), in fully developed turbulent plane channel flow. This exact analysis confirms previous results based on a method-of-images approximation [Manceau R., Wang M., Laurence D.: J. Fluid Mech. 438 (2001) 307-338] showing that, at the wall, $p_{(\mathfrak{V})}^{\prime}$ and $p_{(w)}^{\prime}$ are usually of the same sign and approximately equal. The above results are then used to study the contribution of each mechanism on the pressure correlations in low Reynolds-number plane channel flow, and to discuss standard second-moment-closure modelling practices.

Key Words: turbulence, pressure fluctuations, rapid and slow terms, wall echo, DNs, second-moment closure, Reynolds-stress model

\section{Introduction}

Understanding the physics of turbulent fluctuations of pressure $p^{\prime}$ is of major importance, not only because of their direct implication in noise $(\mathrm{Hu}$ et al. 2002,2006$)$ and excitation of immersed solid surfaces (Corcos 1964), but also because they appear in correlations present in the transport equations for the Reynolds-stresses and the dissipation tensor (Pope 2000; Jovanović 2004). Traditionally the analysis of $p^{\prime}$ is based on the Poisson equation for the fluctuating pressure (Chou 1945), which, at the incompressible flow limit $(\rho \cong$ const $=\bar{\rho} ; \forall t, x$ and $\mu \approx$ const $=\bar{\mu} ; \forall t, x)$, in a nonrotating frame-of-reference, reads

$$
\nabla^{2} p^{\prime}=\nabla^{2}\left(p_{\mathrm{r}}^{\prime}+p_{\mathrm{s}}^{\prime}\right)=\underbrace{-2 \rho \frac{\partial u_{k}^{\prime}}{\partial x_{\ell}} \frac{\partial \bar{u}_{\ell}}{\partial x_{k}}}_{\text {rapid }} \underbrace{-\rho \frac{\partial^{2}}{\partial x_{\ell} \partial x_{k}}\left(u_{k}^{\prime} u_{\ell}^{\prime}-\overline{u_{k}^{\prime} u_{\ell}^{\prime}}\right)}_{\text {slow }}
$$

The incompressible flow Poisson equation (1.1) suggests that (Chou 1945) solenoidal (Hamba 1999) pressure fluctuations, associated with the fluctuating velocity field, are

$\dagger$ Email address for correspondence: isabelle.vallet@upmc.fr 
generated by 2 separate mechanisms $\left(\begin{array}{ll|l|l|l|l|l|l|l|}\hline \text { Rotta } & 1951 a & b & \text { Lumley } 1978 \text {; } & \text { Piquet } 1999 & \text { Pope }\end{array}\right.$ 2000; Jovanović 2004):

(a) the interaction of velocity fluctuations with mean-velocity-gradients called meanflow/turbulence interaction terms, also termed rapid pressure fluctuations, because they interact immediately with an imposed mean-velocity-gradient, or linear pressure fluctuations, because the corresponding source-term in 1.1 is linear in velocity fluctuations, and

(b) the turbulence/turbulence interaction, also termed slow pressure fluctuations, because they react much slower than the rapid ones, which are directly driven by meanvelocity-gradients, or nonlinear pressure fluctuations, because the corresponding sourceterm in (1.1) is quadratic in velocity fluctuations.

This idea of distinguishing between pressure fluctuations associated with mean-flowgradients $\left(p_{(\mathrm{r})}^{\prime}\right)$ and pressure fluctuations associated with turbulence/turbulence interactions only $\left(p_{(\mathrm{s})}^{\prime}\right)$ can be applied in general to all correlations which contain the fluctuating pressure (Rotta $1951 a \mid b$; Lumley 1978, Hanjalić 1994; Piquet 1999; Pope 2000, Jovanović $2004)$.

Chou (1945) pointed out that, because of the linearity in $p^{\prime}$ of the incompressible flow Poisson equation (1.1), separate solutions can be obtained for each of the 2 source-terms, based on the freespace Green's function for the Poisson equation (Katz \& Plotkin 1991)

$$
\begin{aligned}
& p_{(\mathrm{r})}^{\prime}(\vec{x}, t)=\underbrace{\frac{1}{2 \pi} \iiint_{\mathfrak{V}}\left(\rho \frac{\partial \overline{\mathfrak{u}}_{\ell}}{\partial \mathfrak{x}_{k}} \frac{\partial \mathfrak{u}_{k}^{\prime}}{\partial \mathfrak{x}_{\ell}}\right) \frac{d \mathfrak{v}(\overrightarrow{\mathfrak{x}}, t)}{|\overrightarrow{\mathfrak{x}}-\vec{x}|}}_{p_{(\mathrm{r} ; \mathfrak{V})}^{\prime}(\vec{x}, t)} \\
&+\underbrace{\frac{1}{4 \pi} \iint_{\partial \mathfrak{V}}\left(\frac{1}{|\overrightarrow{\mathfrak{x}}-\vec{x}| t)} \frac{\partial \mathfrak{p}_{\mathrm{r}}^{\prime}}{\partial \mathfrak{n}}-\mathfrak{p}_{\mathrm{r}}^{\prime} \frac{\partial}{\partial \mathfrak{n}}\left[\frac{1}{|\overrightarrow{\mathfrak{x}}-\vec{x}|}\right]\right) d \mathfrak{S}(\overrightarrow{\mathfrak{x}}, t)}_{p_{(\mathrm{s})}^{\prime}(\vec{x} ; w)} \\
&=\underbrace{\frac{1}{4 \pi} \iiint_{\mathfrak{V}}\left(\rho \frac{\partial^{2} \mathfrak{u}_{k}^{\prime} \mathfrak{u}_{\ell}^{\prime}}{\partial \mathfrak{x}_{\ell} \partial \mathfrak{x}_{k}}-\rho \frac{\partial^{2} \overline{\mathfrak{u}_{k}^{\prime} \mathfrak{u}_{\ell}^{\prime}}}{\partial \mathfrak{x}_{\ell} \partial \mathfrak{x}_{k}}\right) \frac{d \mathfrak{v}(\overrightarrow{\mathfrak{x}}, t)}{|\overrightarrow{\mathfrak{x}}-\vec{x}|}}_{p_{(\mathrm{s} ; \mathfrak{V})}^{\prime}(\vec{x}, t)} \\
& \underbrace{\frac{1}{4 \pi} \iint_{\partial \mathfrak{V}}\left(\frac{1}{|\overrightarrow{\mathfrak{x}}-\vec{x}|} \frac{\partial \mathfrak{p}_{\mathrm{s}}^{\prime}}{\partial \mathfrak{n}}-\mathfrak{p}_{\mathrm{s}}^{\prime} \frac{\partial}{\partial \mathfrak{n}}\left[\frac{1}{|\overrightarrow{\mathfrak{x}}-\vec{x}|}\right]\right) d \mathfrak{S}(\overrightarrow{\mathfrak{x}}, t)}_{p_{(\mathrm{s} ; w)}^{\prime}(\vec{x}, t)}
\end{aligned}
$$

where $p(\vec{x}, t)$ and $\vec{u}(\vec{x}, t)$ are the pressure and velocity at point $\vec{x}$, and the volume $d \mathfrak{v}(\overrightarrow{\mathfrak{x}}, t)$ and surface $d \mathfrak{S}(\overrightarrow{\mathfrak{x}}, t)$ integrals are taken over all other points $\overrightarrow{\mathfrak{x}}$ where the pressure and velocity are $\mathfrak{p}(\overrightarrow{\mathfrak{x}}, t)$ and $\overrightarrow{\mathfrak{u}}(\overrightarrow{\mathfrak{x}}, t)$. Notice that if $(1.2)$ are multiplied by a function of $\vec{x}$, this function can be entered into the integrals which are over $\overrightarrow{\mathfrak{x}}$. In the case of unbounded flow, where $\partial \mathfrak{V}$ is very far (at infinity), only the volume integrals remain. On the other hand, for flow near solid boundaries, the surface integrals indicate that the unsteady pressure field reacts to the presence of the wall (surface integral; $\mathfrak{n}$ is the normal-to-thewall coordinate, directed outwards from the fluid volume $\mathfrak{V}$ ). Terms related to the surface integrals are usually called wall-echo terms (Hanjalić 1994), since for an isolated infinite plane solid boundary they can be related to reflection from the wall, using the method of images (Shir 1973: Launder et al. 1975, Gibson \& Launder 1978, Piquet 1999: Pope 2000: Jovanović 2004). Wall-echo terms, as defined by the surface integrals in (1.2), contain 
the entire effect of the wall on the pressure fluctuations produced by the inhomogeneous velocity field (already influenced by the wall), and cannot distinguish between different mechanisms by which the presence of the wall affects turbulence (Hunt \& Graham 1978), whose precise identification would require further specific decomposition (projection) of the DNS-computed velocity field (Marmanis 1998, Perot 1999).

Starting from the seminal paper of Chou (1945) all models (Rotta 1951a b; Lumley 1978; Piquet 1999: Pope 2000 Jovanović 2004) for the redistribution tensor $\phi_{i j}$, the velocity/pressure-gradient tensor $\Pi_{i j}$ or the pressure transport $\overline{p^{\prime} u_{i}^{\prime}}$ appearing in the pressure-diffusion tensor $d_{i j}^{(p)}$, are traditionally composed of 4 parts corresponding to the splitting $p^{\prime}=p_{(\mathrm{r} ; \mathfrak{V})}^{\prime}+p_{(\mathrm{r} ; w)}^{\prime}+p_{(\mathrm{s} ; \mathfrak{V})}^{\prime}+p_{(\mathrm{s} ; w)}^{\prime} 1.2$. There is at present no possibility to separately measure $p_{(\mathrm{r} ; \mathfrak{V})}^{\prime}, p_{(\mathrm{r} ; w)}^{\prime}, p_{(\mathrm{s} ; \mathfrak{V})}^{\prime}$, and $p_{(\mathrm{s} ; w)}^{\prime}$, and, despite advances in experimental techniques for the measurement of $p^{\prime}$ (Tsuji et al. 2007; Tsuji \& Ishihara 2003), the simultaneous measurement of $p^{\prime}$ and $\partial_{x_{j}} u_{i}^{\prime}$ in the the wall-vicinity is a difficult challenge (Naka et al. 2006). Direct numerical simulation (DNS) offers the possibility to directly compute the different terms, experimental uncertainty being replaced by the eventual influence of finite size of the computational box and of convergence of statistics.

$\operatorname{Kim}(1989)$, in the context of pseudospectral DNS of incompressible plane channel flow (Kim et al. 1987; Moser et al. 1999), used a Green's function approach (Ince 1926; Courant \& Hilbert 1953; Bender \& Orszag 1978; Ockendon et al. 2003: Zauderer 2006; Myint-U \& Debnath 2007) to solve, as a function of $y$ (normal-to-the-wall coordinate), the incompressible flow Poisson equation for $p^{\prime}(1.1)$, for each parallel-to-the-wall wavenumber $\kappa_{x}$ and $\kappa_{z}$ 丹 The separate solution for each source-term (1.1), permits the separation of slow $p_{(\mathrm{s})}^{\prime}$ and rapid $p_{(\mathrm{r})}^{\prime}$ parts. In this way $(\mathrm{Kim} 1989$, Chang et al. 1999), the slow and rapid contributions to $\phi_{i j}$ were computed (Mansour et al. 1988). This procedure (Kim 1989 ) is now used in a standard way in incompressible plane channel flow DNs, at least as far as $\overline{p_{(\mathrm{s})}^{\prime 2}}, \overline{p_{(\mathrm{r})}^{\prime 2}}$, and $\overline{p_{(\tau)}^{\prime 2}}$ are concerned, and has also been extended to compressible flow studies $($ Foysi et al. 2004$)$. The term $\overline{p_{(\tau)}^{\prime 2}}$, usually called Stokes pressure (Mansour et al. 1988, Chang et al. 1999), corresponds to the separately computed contribution of the wall-boundary condition (Pope 2000, (11.173), p. 439) $\left[\partial_{y} p^{\prime}\right]_{w}=\left[\mu \partial_{y y}^{2} v^{\prime}\right]_{w}, y$ being the normal-to-the-wall direction. Notice that this boundary-condition is associated with the source-term $\partial_{x_{i} x_{i}}^{2}\left[\tau_{i j}^{\prime}\right]$ in the Poisson equation for $p^{\prime}$, which is equal to 0 at the incompressible flow limit (Kim et al. 1987, Moser et al. 1999; Hu \& Sandham 2001; Hoyas \& Jiménez 2006). This procedure was used by Chang et al. (1999) to study the detailed contributions from different locations in the flowfield to wall-pressure wavenumber-frequency spectra.

Manceau et al. (2001) have studied the wall-echo problem from the specific point-ofview of the so-called elliptic relaxation approach of Durbin (1993), which lumps together all wall-echo effects (both on pressure correlations and on dissipation-rate) into a single tensor. These authors (Manceau et al. 2001) used 2-point correlations (in the physical space) from the DNS data of Moser et al. (1999), at wall-friction Reynolds-number $R e_{\tau_{w}}=590$. The method used, which is related to classical work on wall-pressure spectra (Panton \& Linebarger 1974) in that it uses 2-point correlations in physical space, introduces wall-echo by an approximate method of images, one image-channel for the upper

$\dagger$ Throughout the paper $x$ denotes the streamwise coordinate with corresponding velocitycomponent $u, y$ denotes the normal-to-the-wall coordinate with corresponding velocity-component $v$, and $z$ denotes the spanwise coordinate with corresponding velocity-component $w$. Finally, $y=0$ at the channel centerline, and the nondimensional distance from the wall is defined as $y^{+}:=\left(y-y_{w}\right) \bar{u}_{\tau} \nu^{-1}$, where $u_{\tau}$ is the friction-velocity and $\nu$ the kinematic viscosity. 
wall and another for the lower wall. Furthermore, Manceau et al. (2001) neglect Stokes pressure. When analyzed under a formal Green's function framework, the approximation in the method of images used in Manceau et al. (2001) is equivalent to using an approximate Green's function, instead of the exact one. By comparison with the exact solution obtained in the present work it is possible to quantify the approximation error of the method of images.

All known second-moment closures (SMCs) for wall-bounded flows treat pressure correlations appearing in Reynolds-stress transport equations by separating them into a quasi-homogeneous part and a second part accounting for inhomogeneity and/or wallecho. Concerning the quasi-homogeneous part of the redistribution term, general tensorial representations are available (Ristorcelli et al. 1995, Craft \& Launder 1996, Jakirlić \& Hanjalić 2002, Gerolymos et al. 2012a) which in order to satisfy the two-component limit (TCL) realizability constraint (Shih \& Lumley 1993 , turbulence tends to TCL as the wall is approached because of the strong damping of velocity fluctuations normal to the wall) should use representation coefficients 7 that are function of the Reynolds-stress anisotropy tensor invariants (Lumley 1978).

Concerning inhomogeneity and wall-echo, these two mechanisms are not necessarily identical. Wall-echo, which is related to the surface integrals in 1.2 , diminishes with distance-from-the-wall, whereas turbulence-inhomogeneity effects may be active away from the wall, and even in the absence of walls. Indeed comparison of models including inhomogeneity-based correction (Craft \& Launder 1996, Gerolymos \& Vallet 2001; Suga 2004), and of models using wall-topology-based echo-terms, suggests that inhomogeneitybased terms are active at the edge of the boundary-layer and improve the prediction of boundary-layer entrainment. According to the way wall-proximity is handled, sMCs can be classified into three categories

(a) Models which use wall-topology based (for instance distance from the wall or geometric normal to the wall) corrections of the quasi-homogeneous closures, tend to the homogeneous limit away from the wall $\left(y^{+} \gtrsim 30\right)$. Notice that elliptic blending models (Manceau \& Hanjalić 2002) use the same family of tensorial representations for the redistribution $\phi_{i j}$, the velocity/pressure-gradient correlation $\Pi_{i j}$ or the dissipation $\varepsilon_{i j}$ terms, and introduce the knowledge of wall-topology via the boundary conditions of the scalar Helmholtz equation used to define the scalar blending parameter. Therefore, it can be argued that elliptic blending models belong to this category.

(b) Models free from any wall-topology parameters, which use terms based on gradients of local turbulence quantities in the tensorial representations for $\phi_{i j}, \Pi_{i j}$ or $\varepsilon_{i j}$, and are therefore active both near and away from the wall. These models include wall-normalfree models based on a unit-vector in the direction of inhomogeneity $\vec{e}_{\mathrm{I}}($ Gerolymos \& Vallet 2001, Gerolymos et al. 2004), the proposal of Launder \& Li (1994) to Taylorexpand the mean-velocity gradient in $1.2 a$ leading to terms containing $\partial_{x_{j} x_{k}}^{2} \bar{u}_{i}$ in the tensorial representation for $\phi_{i j}$, and the work of Cormack (1975) who suggested the use of extended tensorial representations including gradients of $u_{i}^{\prime} u_{j}^{\prime}$ and of $\varepsilon_{i j}$. This last proposal (Cormack 1975) has not been applied to the development of a working model yet, and recent work (Gerolymos et al. $2012 b)$ suggests that further terms can be profitably added to Cormack's basis.

(c) The full (tensorial) elliptic relaxation approach of Durbin (1993) where all wallaffected terms (both inhomogeneous and wall-echo) are lumped together into a correc-

$\dagger$ The early closures (Rotta 1951a Launder et al. 1975. Speziale et al. 1991$)$ which use constant coefficients do not satisfy the two-component realizability constraint (Shih \& Lumley 1993: Hanjalić 1994, Schwarz \& Bradshaw 1994) 
tion of the homogeneous closures which is obtained by the solution of six Helmholtz equations, one for each component of the Reynolds-stress tensor $\overline{u_{i}^{\prime} u_{j}^{\prime}}$. This approach is fundamentally different from elliptic blending in that it postulates no particular tensorial representation for the near-wall corrections but, instead, determines these via appropriately chosen wall boundary conditions for the Helmholz equations. Unfortunately, very few applications of this approach are available in the literature, where, for complex flows a simplified three-equation closure version is used instead (Durbin 1995). On the other hand, despite statements of the contrary, this approach uses turbulent Reynolds number near-wall dampings 团

None of the above three approaches is perfectly consistent with flow physics, which, ideally would require an inhomogeneous (including gradients of the Reynolds-stresses) tensorial representation coupled to a wall-echo correction.

In the present work we are interested in determining the wall-effects on correlations containing the fluctuating pressure. We aim both at developing a methodology for creating databases of the contribution of each mechanism to $\phi_{i j}, \Pi_{i j}$ and $\overline{p^{\prime} u_{i}^{\prime}}$, and at highlighting the differences between volume ( $\mathfrak{V})$, wall $(w)$, homogeneous (H) and inhomogeneous (I) contributions, The first two contributions are extracted from the DNS computations, while the latter two are used in modelling. Homogeneous terms are not volume terms. They would correspond to hypothetical values of the pressure correlations of a series of independent homogeneous flows, each corresponding to the local values of anisotropy, lengthscale and mean-velocity-gradients. These homogeneous terms are approximately equal to the volume terms away from the wall. Whether such hypothetical homogeneous flows are obtainable for the anisotropy observed near the wall, in pure shear, is an open question.

In $\$ 2$, after a brief description of the present DNS calculations (2.1 and Appendix A, we revisit the Green's function ( 2.2 approach (Kim 1989) applied to DNs computations of incompressible plane channel flow (2.1), and we develop a procedure for separately evaluating $(\$ 2.2 .3)$ the volume $\left(p_{(\mathrm{r} ; \mathfrak{V})}^{\prime}\right.$ and $\left.p_{(\mathrm{s} ; \mathfrak{V})}^{\prime}\right)$ and the wall-echo $\left(p_{(\mathrm{r} ; w)}^{\prime}\right.$ and $\left.p_{(\mathrm{s} ; w)}^{\prime}\right)$ terms in (1.2). By comparison with these exact results we also quantify the error of the approximate method of images of Manceau et al. (2001), as a function of the wavenumber (2.2.4), showing that this method is a high-wavenumber approximation. Analytical details on the Green's functions, with particular emphasis on the singular problem of $x z$-constant pressure fluctuations $(\kappa=0)$, are given in Appendix B. In $\$ 3$ we apply the algorithm ( $(2.2)$ for computing the $p^{\prime}$-splitting 1.2 to low Reynolds-number plane channel flow and study the contribution of different terms to correlations containing $p^{\prime}$.

$\ddagger$ Indeed, the Kolmogorov-scale clippings of the elliptic relaxation lengthscale $L_{\mathrm{ER}}$ (Durbin 1993. (9), p. 469) and timescale $T_{\mathrm{ER}}$ (Durbin 1993, (6), p. 468) can be rewritten as

$$
\begin{aligned}
& T_{\mathrm{ER}}:=\max \left[\frac{\mathrm{k}}{\varepsilon}, C_{T_{\mathrm{ER}}} \sqrt{\frac{\nu}{\varepsilon}}\right]=\max \left[1, C_{T_{\mathrm{ER}}} R e_{\mathrm{T}}^{-\frac{1}{2}}\right] \frac{\mathrm{k}}{\varepsilon} \\
& L_{\mathrm{ER}}:=C_{L_{\mathrm{ER}}} \max \left[\frac{\mathrm{k}^{\frac{3}{2}}}{\varepsilon}, C_{\eta_{\mathrm{ER}}}\left(\frac{\nu^{3}}{\varepsilon}\right)^{\frac{1}{4}}\right]=C_{L_{\mathrm{ER}}} \max \left[1, C_{\eta_{\mathrm{ER}}} R e_{\mathrm{T}}^{-\frac{3}{4}}\right] \frac{\mathrm{k}^{\frac{3}{2}}}{\varepsilon}
\end{aligned}
$$

Hence the switches in $L_{\mathrm{ER}}$ and $T_{\mathrm{ER}}$ used in elliptic relaxation are in essence $R e_{\mathrm{T}}$-dampings (where $R e_{\mathrm{T}}:=\mathrm{k}^{2} \varepsilon^{-1} \nu^{-1}$ is the turbulence Reynolds-number, $\mathrm{k}$ is the turbulence kinetic energy, $\varepsilon$ is its dissipation-rate, and $\nu$ is the kinematic viscosity), expectedly, since the ratios of large-to-Kolmogorov scales are invariably equal to some power of $R e_{\mathrm{T}}$ (Davidson 2004, p. 20). 

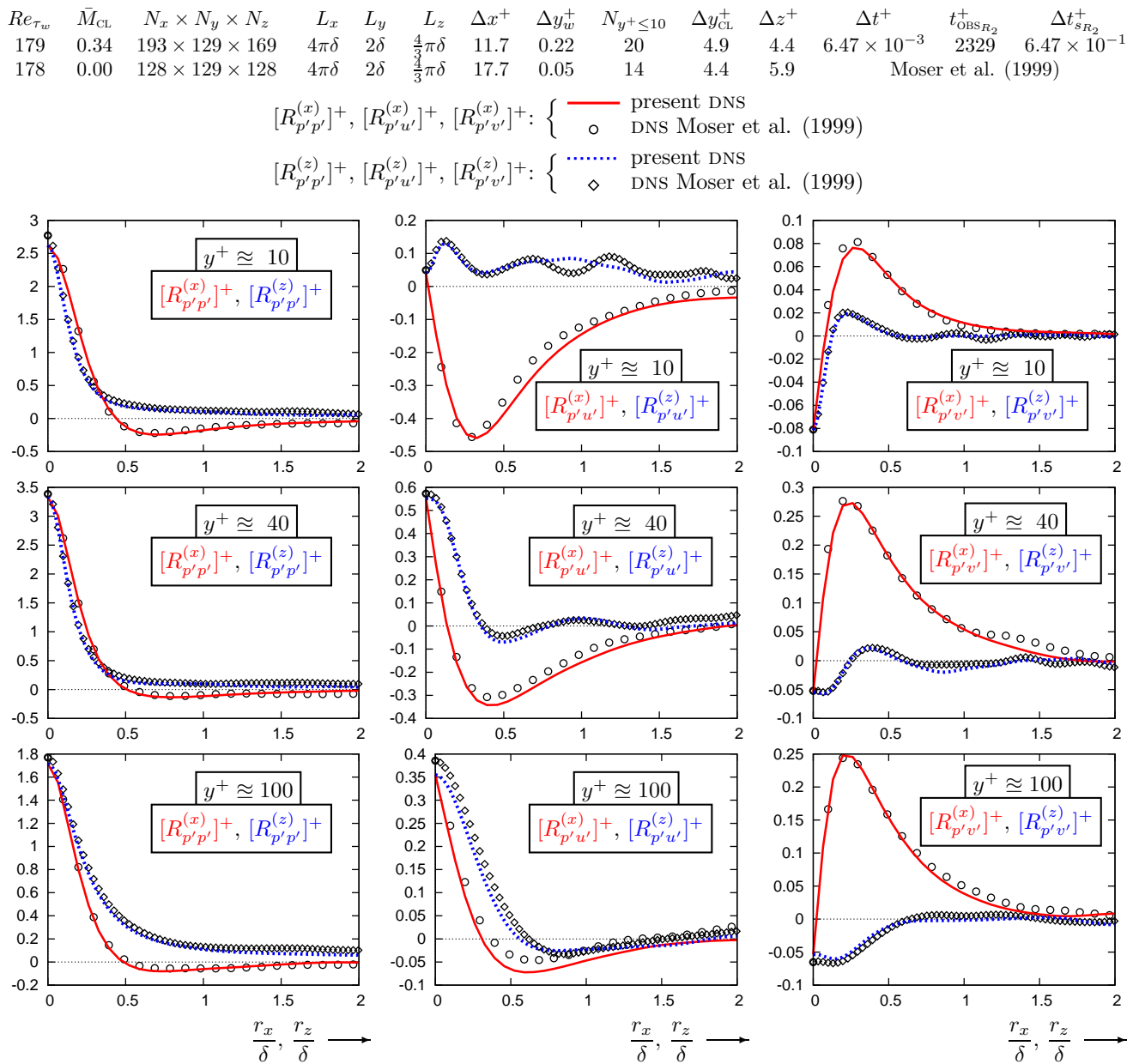

FiguRE 1. Comparison of present DNS-computed $\left(R e_{\tau_{w}}=179 ; \bar{M}_{\mathrm{CL}}=0.34 ; 193 \times 129 \times 169\right.$ grid; Tab. 11 2-point correlations 2.1 containing the fluctuating pressure in wall-scaling $\left(\left[R_{p^{\prime} p^{\prime}}^{(x)}\right]^{+}\right.$, $\left[R_{p^{\prime} u^{\prime}}^{(x)}\right]^{+}$, and $\left[R_{p^{\prime} v^{\prime}}^{(x)}\right]^{+}$in the homogeneous streamwise $(x)$ direction; $\left[R_{p^{\prime} p^{\prime}}^{(z)}\right]^{+},\left[R_{p^{\prime} u^{\prime}}^{(z)}\right]^{+}$, and $\left[R_{p^{\prime} v^{\prime}}^{(z)}\right]^{+}$in the homogeneous spanwise $(z)$ direction), as a function of distance in outer scaling $\left(\delta^{-1} r_{x}\right.$ and $\left.\delta^{-1} r_{z}\right)$, with reference results of incompressible pseudospectral (Kim et al. 1987) DNS computations (Moser et al. 1999, $R e_{\tau_{w}}=178, M_{\mathrm{CL}}=0$ ).

\section{DNS computations and $p^{\prime}$-splitting}

The DNs computations used to obtain the results analyzed in the present paper are discussed in $\$ 2.1$, and the $p^{\prime}$-splitting methodology is described in $\$ 2.2$.

\subsection{Plane channel flow configuration and computational method}

In the particular case of plane channel flow, the boundary surface consists of the upper and lower walls ( $\mathfrak{W}_{l}$ at $y=-\frac{1}{2} L_{y}=-\delta$ and $\mathfrak{W}_{u}$ at $y=+\frac{1}{2} L_{y}=+\delta$, respectively), and the periodic boundaries at $x= \pm \frac{1}{2} L_{x}$ and $z= \pm \frac{1}{2} L_{z}$. The methodology described in the present paper is independent of the particular DNS solver used. The DNs database was generated for $R e_{\tau_{w}}:=\bar{u}_{\tau} \delta \nu^{-1}=180$ (where $2 \delta=L_{y}$ is the channel height, $u_{\tau}:=\sqrt{\bar{\tau}_{w} \rho^{-1}}$ is the friction velocity and $\nu$ the kinematic viscosity) using the DNS solver described and validated in Gerolymos et al. (2010), which solves the flow-equations in physical 


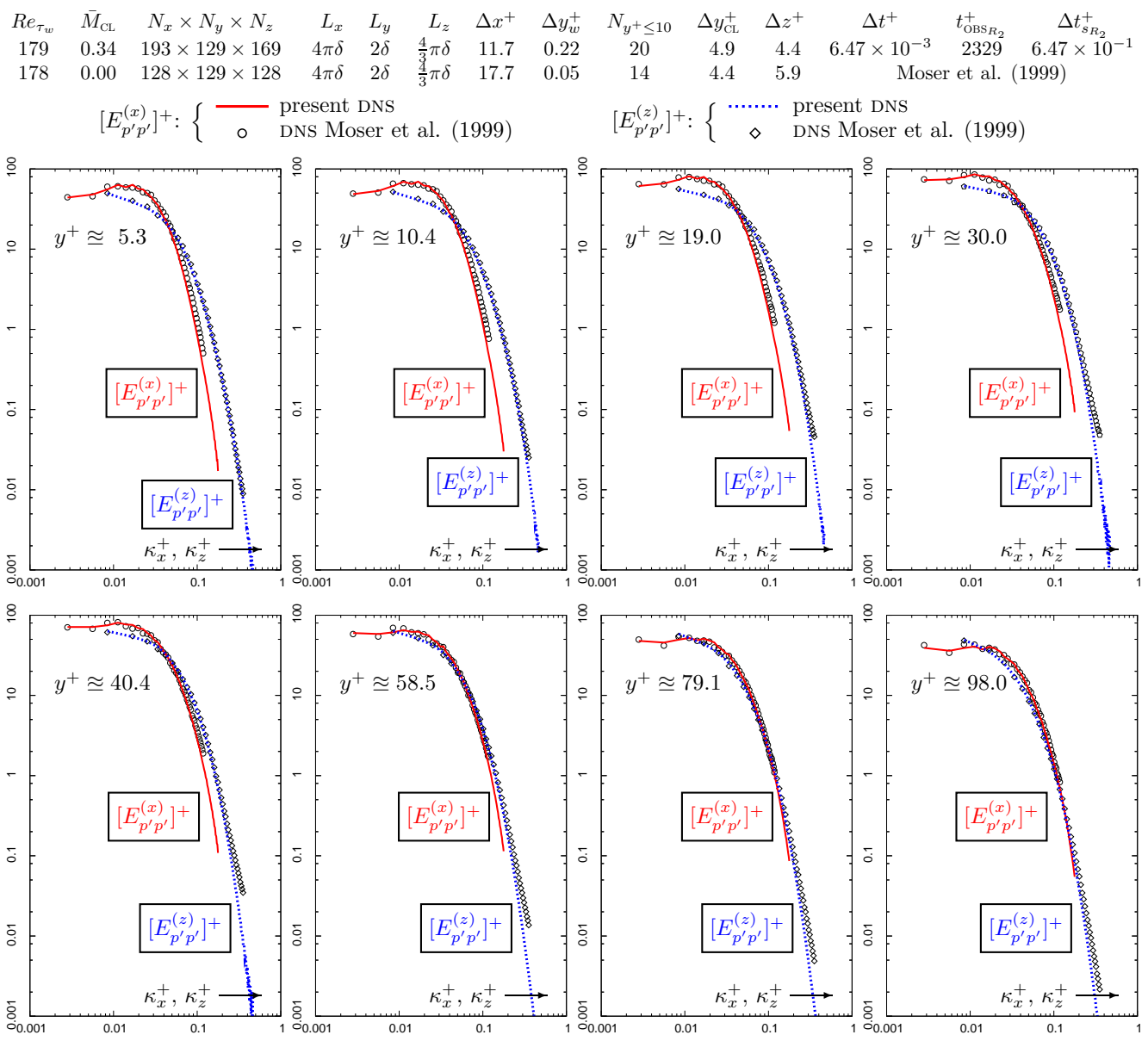

FiguRE 2. Comparison of present DNS-computed $\left(R e_{\tau_{w}}=179 ; \bar{M}_{\mathrm{CL}}=0.34 ; 193 \times 129 \times 169\right.$ grid; Tab. 1 1-D pressure spectra in wall scaling $\left(\left[E_{p^{\prime} p^{\prime}}^{(x)}\right]^{+}\right.$and $\left[E_{p^{\prime} p^{\prime}}^{(z)}\right]^{+}$in the homogeneous streamwise $(x)$ and spanwise $(z)$ directions), as a function of wavenumber in wall units ( $\kappa_{x}^{+}$and $\kappa_{z}^{+}$), with reference results of incompressible pseudospectral (Kim et al. 1987) DNs computations (Moser et al. 1999, $\left.R e_{\tau_{w}}=178, M_{\mathrm{CL}}=0\right)$ ).

space, with $O\left(\Delta x^{17}\right)$ discretization of the convective terms (Gerolymos et al. 2009). The flow is modelled by the compressible Navier-Stokes equations with air as working medium, but for the quasi-incompressible $\bar{M}_{\mathrm{CL}} \approx 0.34$ Mach-number considered in the present work mean density $\bar{\rho}$ variations do not exceed $1.5 \%$, and density fluctuations are negligibly small $\left(\rho_{\text {rms }}^{\prime} \lesssim 0.25 \% \bar{\rho}\right)$. For this reason the turbulent correlations obtained can be considered as incompressible. This has been verified by systematic comparison with standard incompressible pseudospectral DNS data (Kim et al. 1987; Moser et al. 1999: del Álamo \& Jiménez 2003; del Álamo et al. 2004, Hoyas \& Jiménez 2006, 2008), both for single point statistics (Gerolymos et al.|2010, all soms $\dagger$ and TOMs\$ appearing in the Reynolds-stress budgets) and for spectra of velocity fluctuations in the homogeneous

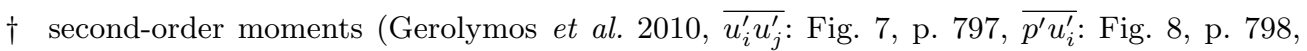
$\phi_{i j}:=2 \overline{p^{\prime} S_{i j}^{\prime}}:$ Fig. 10, p. 800, $\varepsilon_{i j}^{(\mu)}:=2 \nu \overline{\partial_{x_{\ell}} u_{i}^{\prime} \partial_{x_{\ell}} u_{j}^{\prime}}:$ Fig. 11, p. 801) 


$\begin{array}{ccccccccccccccc}R e_{\tau_{w}} & \bar{M}_{\mathrm{CL}} & N_{x} \times N_{y} \times N_{z} & L_{x} & L_{y} & L_{z} & \Delta x^{+} & \Delta y_{w}^{+} & N_{y}+\leq 10 & \Delta y_{\mathrm{CL}}^{+} & \Delta z^{+} & \Delta t^{+} & t_{\mathrm{OBS}}^{+} & \Delta t_{s}^{+} & \Delta t_{s_{2}}^{+} \\ 179 & 0.34 & 193 \times 129 \times 169 & 4 \pi \delta & 2 \delta & \frac{4}{3} \pi \delta & 11.7 & 0.22 & 20 & 4.9 & 4.4 & 6.47 \times 10^{-3} & 2329 & 6.47 \times 10^{-3} & 6.47 \times 10^{-1} \\ 180 & 0.34 & 257 \times 129 \times 385 & 8 \pi \delta & 2 \delta & 4 \pi \delta & 17.6 & 0.23 & 20 & 5.0 & 5.9 & 6.56 \times 10^{-3} & 289 & 6.57 \times 10^{-3} & 6.57 \times 10^{-1} \\ 180 & 0.34 & 129 \times 129 \times 129 & 4 \pi \delta & 2 \delta & \frac{4}{3} \pi \delta & 17.7 & 0.23 & 20 & 5.0 & 5.9 & 6.56 \times 10^{-3} & 1539 & 6.56 \times 10^{-3} & 6.56 \times 10^{-1} \\ 184 & 0.34 & 121 \times 161 \times 81 & 4 \pi \delta & 2 \delta & \frac{4}{3} \pi \delta & 19.3 & 0.19 & 25 & 4.4 & 9.6 & 5.50 \times 10^{-3} & 1014 & 5.50 \times 10^{-3} & 5.50 \times 10^{-1}\end{array}$

TABle 1. Parameters of the DNS computations $\left[L_{x}, L_{y}, L_{z}\left(N_{x}, N_{y}, N_{z}\right)\right.$ are the dimensions (number of grid-points) of the computational domain $(x=$ homogeneous streamwise, $y=$ normal-to-the-wall, $z=$ homogeneous spanwise direction); $\delta$ is the channel halfheight; $\Delta x^{+}, \Delta y_{w}^{+}$, $\Delta y_{\mathrm{CL}}^{+}, \Delta z^{+}$are the mesh-sizes in wall-units; $(\cdot)_{w}$ denotes wall and $(\cdot)_{\mathrm{CL}}$ centerline values; $N_{y^{+} \leq 10}$ is the number of grid points between the wall and $y^{+}=10 ; R e_{\tau_{w}}:=\bar{u}_{\tau} \delta \bar{\nu}_{w}^{-1} ; \bar{u}_{\tau}$ is the friction velocity; $\delta$ is the channel halfheight; $\bar{\nu}_{w}=$ is the kinematic viscosity at the wall; $\bar{M}_{\mathrm{CL}}$ is the centerline Mach-number; $\Delta t^{+}$is the computational time-step in wall-units; $t_{\mathrm{OBS}}^{+}$is the observation period in wall units over which statistics were computed; $\Delta t_{s}^{+}$is the sampling time-step for the single-point statistics in wall-units; $\Delta t_{s_{R_{2}}}^{+}$is the sampling time-step for the two-point statistics in wall-units].

streamwise and spanwise directions (Gerolymos et al. 2010, Figs. 12-15, pp. 802-805). This very good agreement with pseudospectral incompressible DNS (Kim et al. 1987, Hoyas \& Jiménez 2008) is also valid for 2-point correlations

$$
R_{\mathrm{a}^{\prime} \mathrm{b}^{\prime}}(\vec{x}, \vec{r}):=\overline{\mathrm{a}^{\prime}(\vec{x}, t) \mathrm{b}^{\prime}(\vec{x}+\vec{r}, t)}
$$

containing the fluctuating pressure $p^{\prime}$, viz $\left[R_{p^{\prime} p^{\prime}}^{(x, z)}\right]^{+},\left[R_{p^{\prime} u^{\prime}}^{(x, z)}\right]^{+}$, and $\left[R_{p^{\prime} v^{\prime}}^{(x, z)}\right]^{+}$(Fig. 11 and fluctuating pressure spectrat $\left[E_{p^{\prime} p^{\prime}}^{(x)}\right]^{+}$and $\left[E_{p^{\prime} p^{\prime}}^{(z)}\right]^{+}$(Fig. 2).

In order to evaluate more precisely how the small compressibility in the DNS computations affects the fluctuating pressure field, an order-of-magnitude analysis of the complete compressible flow equation for $\nabla^{2} p^{\prime}$ (A 1d) was performed (Appendix A), indicating that the additional compressible terms in (A1d) scale with density fluctuations and gradients (A 7), and may be reasonably neglected for the present flow conditions (Fig. A1). The main effect of compressibility in the present computations, as far as $p^{\prime}$-splitting is concerned, comes from the variations of mean density $\bar{\rho}(\lesssim 1.5 \%)$, which appears in the source-terms $Q_{(\mathrm{s})}^{\prime}+Q_{(\mathrm{r})}^{\prime} \sqrt{1.1}$ A 1d), in line with Morkovin's hypothesis (So et al. 1998).

The influence of the Reynolds-number needs consideration, especially as the data used in the present computations correspond to a rather low $R e_{\tau_{w}} \approx 180$. It is well known that wall-pressure fluctuations, in wall-units, $\left[p_{w}^{\prime}\right]_{\mathrm{rms}}^{+}$, show a marked influence on Reynoldsnumber (Goody 2004, Hu et al. 2006; Tsuji et al. 2007), although alternative scalings exhibit a less pronounced dependence (Tsuji et al. 2007, Fig. 14, p.21). However, the ratio of slow-to-rapid pressure fluctuations $\left[p_{(r)}^{\prime}\right]_{\mathrm{rms}}^{-1}\left[p_{(s)}^{\prime}\right]_{\mathrm{rms}}$ (Fig. 3p shows little dependence on $R e_{\tau_{w}}$ for $y^{+} \lesssim 100$, ranging from $\sim 0.9$ at the wall to $\sim 1.6$ at $y^{+} \approx 50$, and is approximately constant $\sim 1.6$ in the region $\frac{2}{10} \delta \lesssim y-y_{w} \lesssim \frac{7}{10} \delta$ (Fig. 3). At and near the centerline $y=\delta$, there is some scatter between different $R e_{\tau_{w}}$, but no clear trend is discernible (Fig. 3). These results, based on the DNs data of Hoyas \& Jiménez (2008), suggest that, although $p^{\prime}$ exhibits strong dependence on $R e_{\tau_{w}}$, the relative importance of the slow and rapid mechanisms in the creation of $p^{\prime}$ does not. Therefore, despite the low

$\dagger$ Notice that the spectra given by Moser et al. $(1999)$ are twice the DFT of $R_{p^{\prime} p^{\prime}}^{+}$(in wall units), and have to be appropriately rescaled (Briggs \& Henson 1995$)$ by $(2 \pi)^{-1} L_{x}^{+}$or $(2 \pi)^{-1} L_{z}^{+}$ to get actual 1-D spectra $E_{u_{i} u_{j}}^{+}$(Pope 2000, (6.206), p. 225), as those given by del Álamo et al. (2004). 


$\begin{array}{rrcrrrrrrrrrr}R e_{\tau_{w}} & \bar{M}_{\mathrm{CL}} & N_{x} \times N_{y} \times N_{z} & L_{x} & L_{y} & L_{z} & \Delta x^{+} & \Delta y_{w}^{+} & N_{y^{+} \leq 10} & \Delta y_{\mathrm{CL}}^{+} & \Delta z^{+} & t_{\mathrm{OBS}}^{+} & \Delta t_{s}^{+} \\ 186 & 0.00 & 768 \times 97 \times 340 & 12 \pi \delta & 2 \delta & 4 \pi \delta & 8.9 & 0.10 & 11 & 6.1 & 6.7 & 11740 & 110 \\ 547 & 0.00 & 1536 \times 257 \times 1536 & 8 \pi \delta & 2 \delta & 4 \pi \delta & 8.9 & 0.04 & 16 & 6.7 & 4.5 & 12034 & 81 \\ 934 & 0.00 & 3072 \times 385 \times 2304 & 8 \pi \delta & 2 \delta & 3 \pi \delta & 9.2 & 0.03 & 18 & 7.6 & 3.8 & 11208 & 151 \\ 2003 & 0.00 & 6144 \times 633 \times 4608 & 8 \pi \delta & 2 \delta & 3 \pi \delta & 8.2 & 0.32 & 12 & 8.9 & 4.1 & 22033 & 97\end{array}$
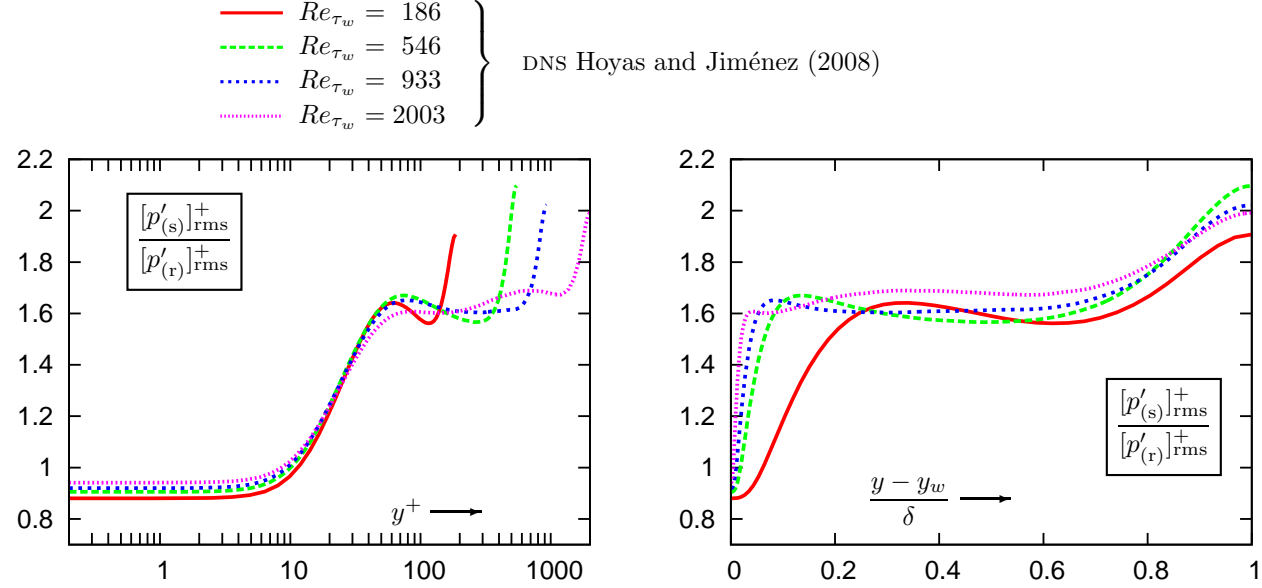

FiguRE 3. Ratio of slow-to-rapid pressure fluctuations $\left[p_{(r)}^{\prime}\right]_{\mathrm{rms}}^{-1}\left[p_{(s)}^{\prime}\right]_{\mathrm{rms}}[1.1$, from the incompressible DNS database of Hoyas \& Jiménez (2006, 2008), for different Reynolds numbers $\left(\operatorname{Re}_{\tau_{w}} \in\{186,547,934,2003\}\right)$, plotted against the nondimensional distance-from-the-wall in inner $\left(y^{+}\right)$and outer $\left(\delta^{-1}\left(y-y_{w}\right)\right)$ scaling.

$R e_{\tau_{w}} \approx 180$ of the present DNS computations, it is believed that the obtained $p^{\prime}$-splitting results provide useful information on the relative importance of different mechanisms in the correlations containing $p^{\prime}$. Nevertheless, DNS data on volume $\left(p_{(\mathrm{r} ; \mathfrak{V})}^{\prime}\right.$ and $\left.p_{(\mathrm{s} ; \mathfrak{V})}^{\prime}\right)$ and wall-echo $\left(p_{(\mathrm{r} ; w)}^{\prime}\right.$ and $\left.p_{(\mathrm{s} ; w)}^{\prime}\right)$ terms at higher $R e_{\tau_{w}}$ are required to substantiate this.

\subsection{Green's function solution of the Poisson equations}

For fully developed ( $x$-wise invariant in the mean) turbulent plane channel flow, the Poisson equation for $p^{\prime}$ 1.1) can be simplified to a system of independent ODEs, one for each Fourier-component of $p^{\prime}$, which can be efficiently solved by a Green's function approach (Kim 1989). In 2.2.1, we formalize the problem, in relation to previous work. Using mathematical tools and solutions detailed in Appendix B, we discuss, in $\{2.2 .2$, the standard decomposition $p^{\prime}=p_{(\mathrm{r})}^{\prime}+p_{(\mathrm{s})}^{\prime}+p_{(\tau)}^{\prime} 2.2$, with special emphasis on the singular case of the $x z$-averaged component ${\overline{p^{\prime}}}^{x z}(y, t) 2.6 d)$. In 2.2 .3 , we present the new methodology for the identification of volume and wall-echo terms in the decomposition $p^{\prime}=p_{(\mathrm{r} ; \mathfrak{V})}^{\prime}+p_{(\mathrm{r} ; w)}^{\prime}+p_{(\mathrm{s} ; \mathfrak{Y})}^{\prime}+p_{(\mathrm{s} ; w)}^{\prime}+p_{(\tau)}^{\prime} 2.10$. In 2.2 .4 . we show how the method-of-images approach applied to fully developed turbulent plane channel flow, can be represented by an appropriate Green's function. Finally, in $\$ 2.2 .5$, we show that the approximation made in the method of images consists of neglecting the interaction of wall-echo between the 2 walls, and demonstrate by comparison of the exact and approximate Green's functions that this is a high-wavenumber approximation.

\subsubsection{ODEs for the Fourier transforms}

The solution to (1.1), with Neumann boundary-conditions at the walls $(\overline{\text { Pope }} 2000$, p. 439) and periodic boundary-conditions in the homogeneous directions, can only be obtained up to an additive function of time $t$ (Ince 1926, Courant \& Hilbert 1953, Myint- 


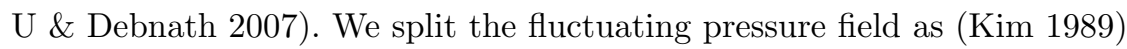

$$
p^{\prime}(x, y, z, t)=p_{(\mathrm{r})}^{\prime}(x, y, z, t)+p_{(\mathrm{s})}^{\prime}(x, y, z, t)+p_{(\tau)}^{\prime}(x, y, z, t)
$$

where the rapid $p_{(\mathrm{r})}^{\prime}$, slow $p_{(\mathrm{s})}^{\prime}$ and Stokes $p_{(\tau)}^{\prime}$ pressure fluctuations are solutions of $(\mathrm{Kim}$ 1989: Chang et al. 1999)

$$
\nabla^{2}\left[\begin{array}{c}
p_{(\mathrm{r})}^{\prime} \\
p_{(\mathrm{s})}^{\prime} \\
p_{(\tau)}^{\prime}
\end{array}\right]=\left[\begin{array}{c}
Q_{(\mathrm{r})}^{\prime} \\
Q_{(\mathrm{s})}^{\prime} \\
Q_{(\tau)}^{\prime}
\end{array}\right]:=\left[\begin{array}{c}
-2 \rho \partial_{x_{\ell}} u_{k}^{\prime} \partial_{x_{k}} \bar{u}_{\ell} \\
-\rho\left(\partial_{x_{\ell}} u_{k}^{\prime} \partial_{x_{k}} u_{\ell}^{\prime}-\bar{\partial}_{x_{\ell}} u_{k}^{\prime} \partial_{x_{k}} u_{\ell}^{\prime}\right.
\end{array}\right]
$$

with boundary-conditions (Pope 2000 , pp. 390-392, 439-442)

$$
\frac{\partial}{\partial y}\left[\begin{array}{c}
p_{(\mathrm{r})}^{\prime} \\
p_{(\mathrm{s})}^{\prime} \\
p_{(\tau)}^{\prime}
\end{array}\right]\left(x, y= \pm \frac{1}{2} L_{y}, z, t\right)=\left[\begin{array}{c}
0 \\
0 \\
B_{(\tau)_{ \pm}}^{\prime}
\end{array}\right]:=\left[\begin{array}{c}
0 \\
0 \\
\mu \partial_{y y}^{2} v^{\prime}
\end{array}\right]
$$

Since 2.3 is linear in $p_{(m)}^{\prime}(m \in\{\mathrm{r}, \mathrm{s}, \tau\})$ the 3 problems can be solved independently to compute the 3 fields in (2.2). The last field in the superposition 2.2 is introduced to satisfy the wall boundary-conditions associated with the normal-to-the-wall $(y)$ momentum equation

$$
\left.\frac{\partial p^{\prime}}{\partial y}\right|_{y= \pm \frac{1}{2} L_{y}}=\left.\frac{\partial \tau_{y \ell}^{\prime}}{\partial x_{\ell}}\right|_{y= \pm \frac{1}{2} L_{y}}=\left.\mu \nabla^{2} v^{\prime}\right|_{y= \pm \frac{1}{2} L_{y}}=\left.\mu \frac{\partial^{2} v^{\prime}}{\partial y^{2}}\right|_{y= \pm \frac{1}{2} L_{y}}
$$

because of the no-slip wall boundary-condition $u_{i}^{\prime}\left(x, y= \pm \frac{1}{2} L_{y}, z, t\right)=0 \forall x, z, t$. By (2.4) the field $p_{(\tau)}^{\prime}$ is obviously related to the fluctuating wall-shear-stress, and is usually called Stokes pressure (Mansour et al. 1988, Kim 1989, Chang et al. 1999) although Pope (2000, p. 439) suggests the alternative term harmonic pressure, because in incompressible flow $\nabla^{2} p_{\tau}^{\prime}=0$ 田

The directions $x$ and $z$ being homogeneous (periodic in the computational model), following $\operatorname{Kim}(1989)$, we replace the $x z$-Fourier-transforms 9 of $p_{(m)}^{\prime}, Q_{(m)}^{\prime}(m \in\{\mathrm{r}, \mathrm{s}, \tau\})$ and $B_{(\tau) \pm}^{\prime}$

$$
\begin{aligned}
p_{(m)}^{\prime}(x, y, z, t) & =\int_{-\infty}^{+\infty} \int_{-\infty}^{+\infty} \hat{p}_{(m)}^{\prime}\left(\kappa_{x}, y, \kappa_{z}, t\right) e^{i \kappa_{x} x+i \kappa_{z} z} d \kappa_{x} d \kappa_{z} \\
Q_{(m)}^{\prime}(x, y, z, t) & =\int_{-\infty}^{+\infty} \int_{-\infty}^{+\infty} \hat{Q}_{(m)}^{\prime}\left(\kappa_{x}, y, \kappa_{z}, t\right) e^{i \kappa_{x} x+i \kappa_{z} z} d \kappa_{x} d \kappa_{z} \\
B_{(\tau)_{ \pm}}^{\prime}(x, z, t) & =\int_{-\infty}^{+\infty} \int_{-\infty}^{+\infty} \hat{B}_{(\tau)_{ \pm}}^{\prime}\left(\kappa_{x}, \kappa_{z}, t\right) e^{i \kappa_{x} x+i \kappa_{z} z} d \kappa_{x} d \kappa_{z}
\end{aligned}
$$

$\dagger$ recall that, for strictly incompressible flow, the continuity equation for the fluctuating velocity field, $\partial_{x_{\ell}} u_{\ell}^{\prime}=0$, implies $\partial_{x_{\ell} x_{k}}^{2}\left(u_{k}^{\prime} u_{\ell}^{\prime}\right)=\partial_{x_{\ell}} u_{k}^{\prime} \partial_{x_{k}} u_{\ell}^{\prime}$

$\ddagger$ This is however no longer true in the compressible flow case (Foysi et al. 2004).

I The Fourier-transforms $\hat{p}_{(m)}^{\prime}, \hat{Q}_{(m)}^{\prime}, \hat{B}_{(m)}^{\prime} \in \mathbb{C}$ are computed using standard DFT (discrete Fourier transform) techniques (Briggs \& Henson 1995) in the periodic directions $x$ and $z$, with maximum computable wavenumbers $\kappa_{x_{\max }}=\pi(\Delta x)^{-1}$ and $\kappa_{z_{\max }}=\pi(\Delta z)^{-1}$. 
in the PDE

$$
\begin{aligned}
& {\left[\frac{\partial^{2}}{\partial y^{2}}-\kappa^{2}\right]\left[\begin{array}{c}
\hat{p}_{(\mathrm{r})}^{\prime} \\
\hat{p}_{(\mathrm{s})}^{\prime} \\
\hat{p}_{(\tau)}^{\prime}
\end{array}\right]\left(\kappa_{x}, y, \kappa_{z}, t\right)=\left[\begin{array}{c}
\hat{Q}_{(\mathrm{r})}^{\prime} \\
\hat{Q}_{(\mathrm{s})}^{\prime} \\
\hat{0}
\end{array}\right]\left(\kappa_{x}, y, \kappa_{z}, t\right)} \\
& \frac{\partial \hat{p}_{(m)}^{\prime}}{\partial y}\left(\kappa_{x}, y= \pm \frac{1}{2} L_{y}, \kappa_{z}, t\right)=\left[\begin{array}{c}
0 \\
0 \\
\hat{B}_{(\tau)_{ \pm}}^{\prime}\left(\kappa_{x}, \kappa_{z}, t\right)
\end{array}\right] \\
& \kappa:=\sqrt{\kappa_{x}^{2}+\kappa_{z}^{2}} \in \mathbb{R}_{\geq 0}
\end{aligned}
$$

The classical solution of 2.6 by Kim (1989), using a Green's function approach, provides detailed information on the structure of the rapid and slow fields, and was extended by Chang et al. (1999) to study the detailed influence of the sources, both in type (slow and rapid) and $y$-location in the channel. In the present work we extend this analysis by identifying and studying the wall-echo influence. Notice that, by 2.5,

$$
\hat{p}^{\prime}\left(\kappa_{x}=0, y, \kappa_{z}=0, t\right) \stackrel{2.5 a}{-}{\overline{p^{\prime}(x, y, z, t)}}^{x z} \in \mathbb{R}
$$

the case $\kappa=0$ corresponds to the time-fluctuation of the $x z$-averaged pressure at each $y$-location, while

$$
p_{\mathrm{B}}^{\prime}(t):={\overline{p^{\prime}(x, y, z, t)}}^{x y z} \frac{1}{2.5 a 22.6 d} \int_{-\frac{1}{2} L_{y}}^{+\frac{1}{2} L_{y}} \hat{p}^{\prime}\left(\kappa_{x}=0, y, \kappa_{z}=0, t\right) d y
$$

is the time-fluctuation of the bulk (volume-averaged) pressure.

\subsubsection{Exact solution of 2.6}

The exact solution to $(2.6 a)$ with boundary conditions $(2.6 b)$, for the $x z$-Fouriercomponents 2.5a) of the slow $p_{(\mathrm{s})}^{\prime}$ and rapid $p_{(\mathrm{r})}^{\prime}$ fields, is given in Kim (1989), and has been widely used (Mansour et al. 1988, Chang et al. 1999; Foysi et al. 2004). Following the analysis in $\$ \mathrm{~B} .2 .1$ for $\kappa \neq 0(\mathrm{~B} 10 b)$ and in $\$ \mathrm{~B} .3 .1$ for $\kappa=0$ (B 28), it reads

$$
\left[\begin{array}{c}
\hat{p}_{(\mathrm{r})}^{\prime} \\
\hat{p}_{(\mathrm{s})}^{\prime}
\end{array}\right]\left(\kappa_{x}, y, \kappa_{z}, t\right)=\int_{-\frac{1}{2} L_{y}}^{+\frac{1}{2} L_{y}} G_{\mathrm{Kim}}(y, Y ; \kappa)\left[\begin{array}{c}
\hat{Q}_{(\mathrm{r})}^{\prime} \\
\hat{Q}_{(\mathrm{s})}^{\prime}
\end{array}\right]\left(\kappa_{x}, Y, \kappa_{z}, t\right) d Y
$$

where the Green's function

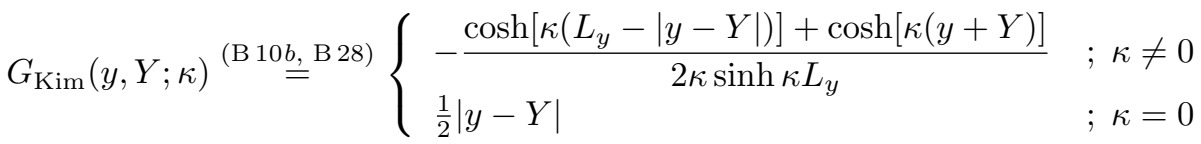

and satisfies the correct homogeneous Neumann boundary-conditions on both walls (Fig. (4). The exact solution to (2.6a), for the $x z$-Fourier-components $(2.5 a)$ of the Stokes (harmonic) pressure, with boundary-conditions (2.6b) was given in Chang et al. (1999), 
and following the analysis in $\$ \mathrm{~B} .2 .2$ for $\kappa \neq 0(\mathrm{~B} 12)$ and in $\$ \mathrm{~B} .3 .2$ for $\kappa=0(\mathrm{~B} 32)$, reads

$$
\begin{aligned}
& \hat{p}_{(\tau)}^{\prime}\left(\kappa_{x}, y, \kappa_{z}, t\right) \\
& \begin{cases}\frac{\hat{B}_{(\tau)_{+}}^{\prime}\left(\kappa_{x}, \kappa_{z}, t\right) \cosh \left[\kappa\left(\frac{1}{2} L_{y}+y\right)\right]-\hat{B}_{(\tau)_{-}}^{\prime}\left(\kappa_{x}, \kappa_{z}, t\right) \cosh \left[\kappa\left(\frac{1}{2} L_{y}-y\right)\right]}{\kappa \sinh \kappa L_{y}} & ; \kappa \neq 0 \\
\frac{1}{2} \hat{B}_{(\tau)_{-}}^{\prime}\left(\kappa_{x}, \kappa_{z}, t\right) y+\frac{1}{2} \hat{B}_{(\tau)_{+}}^{\prime}\left(\kappa_{x}, \kappa_{z}, t\right) y+p_{\mathrm{BC}_{0}}^{\prime}(t) & ; \kappa=0\end{cases}
\end{aligned}
$$

where the choice of the additive constant $p_{\mathrm{BC} 0}^{\prime} \in \mathbb{R}$, up to which the harmonic pressure can be defined ( $\$$ B.3.2), was chosen to satisfy the constraint $p_{\mathrm{B}}^{\prime}(t):={\overline{p^{\prime}}}^{x y z}(t)=0($ ie constant bulk pressure).

As discussed in $\sqrt{\mathrm{B} .3}$, the case $\kappa=0$ is singular, and a solution exists iff the compatibility conditions (B 31, B 25)

$$
\begin{gathered}
L_{y}\left[\begin{array}{c}
\overline{Q_{(\mathrm{r})}^{\prime}} x y z \\
\overline{Q_{(\mathrm{s})}^{\prime}} x y z
\end{array}\right](t)=\int_{-\frac{1}{2} L_{y}}^{+\frac{1}{2} L_{y}}\left[\begin{array}{c}
\hat{Q}_{(\mathrm{r})}^{\prime} \\
\hat{Q}_{(\mathrm{s})}^{\prime}
\end{array}\right]\left(\kappa_{x}=0, Y, \kappa_{z}=0, t\right) d Y=\left[\begin{array}{l}
0 \\
0
\end{array}\right] \\
\hat{B}_{(\tau)_{+}}^{\prime}\left(\kappa_{x}=0, \kappa_{z}=0, t\right)=\hat{B}_{(\tau)_{-}}^{\prime} \frac{\left(\kappa_{x}=0, \kappa_{z}=0, t\right) \Longleftrightarrow}{\overline{B_{(\tau)_{+}}^{\prime}} x z}(t)=\overline{B_{(\tau)_{-}}^{\prime}} x z(t)
\end{gathered}
$$

hold, where $\overline{(\cdot)}^{x y z}$ denotes the volume averag $f$ (bulk average) and $\overline{(\cdot)}^{x z}$ is the surface averag $\oplus$ in the homogeneous directions $x$ and $z$. If the computational box is large enough in the homogeneous directions for the ergodic hypothesis (Monin \& Yaglom 1971, p. 243-256) to hold, (2.9) are satisfied, at least approximately. In practice, the compatibility condition $2.9 a$ was enforced numerically when computing the Fourier-transforms $2.5 b)$. The form (B 32) of $q_{\mathrm{BC}}\left(y ; \kappa=0, B_{ \pm}\right)$automatically enforces the compatibility condition, by taking the average gradient $\frac{1}{2}\left(B_{-}+B_{+}\right)$. In practice $\hat{B}_{(\tau)_{-}}^{\prime}\left(\kappa_{x}=0, \kappa_{z}=0, t\right) \approx$ $\hat{B}_{(\tau)_{+}}^{\prime}\left(\kappa_{x}=0, \kappa_{z}=0, t\right) \approx 0$ in $2.9 b$, especially with increasing box size, so that taking the average is a good choice. Of course, (1.1) with Neumann boundary conditions at the walls (Pope 2000, p. 439), can only be solved up to an additive constant, which is fixed by the choice of $p_{\mathrm{BC}_{0}}^{\prime}$ in the Stokes field $p_{(\tau)}^{\prime}$ 2.8) (ie constant bulk pressure).

\subsubsection{Volume and wall-echo terms}

The main purpose of the present algorithm is to distinguish in 1.2 the contributions of the volume integral (volume terms denoted by $(\cdot)_{\mathfrak{V}}$ ) from the contributions of the surface integral (wall-echo terms denoted by $(\cdot)_{w}$ ), ie to extend 2.2 as

$$
\begin{array}{r}
p^{\prime}(x, y, z, t)=\underbrace{p_{(\mathrm{r} ; \mathfrak{V})}^{\prime}(x, y, z, t)+p_{(\mathrm{r} ; w)}^{\prime}(x, y, z, t)}_{p_{(\mathrm{r})}^{\prime}(x, y, z, t)} \\
+\underbrace{p_{(\mathrm{s} ; \mathfrak{V})}^{\prime}(x, y, z, t)+p_{(\mathrm{s} ; w)}^{\prime}(x, y, z, t)}_{p_{(\mathrm{s})}^{\prime}(x, y, z, t)}+p_{(\tau)}^{\prime}(x, y, z, t) \\
\dagger \overline{(\cdot)}{ }^{x y z}:=\frac{1}{L_{x} L_{y} L_{z}} \int_{-\frac{1}{2} L_{x}}^{+\frac{1}{2} L_{x}} \int_{-\frac{1}{2} L_{y}}^{+\frac{1}{2} L_{y}} \int_{-\frac{1}{2} L_{z}}^{+\frac{1}{2} L_{z}}(\cdot) d x d y d z \\
\overline{(\cdot)} x z:=\frac{1}{L_{x} L_{z}} \int_{-\frac{1}{2} L_{x}}^{+\frac{1}{2} L_{x}} \int_{-\frac{1}{2} L_{z}}^{+\frac{1}{2} L_{z}}(\cdot) d x d z
\end{array}
$$




$$
G_{\mathrm{MWL}}(y, Y ; \kappa)
$$
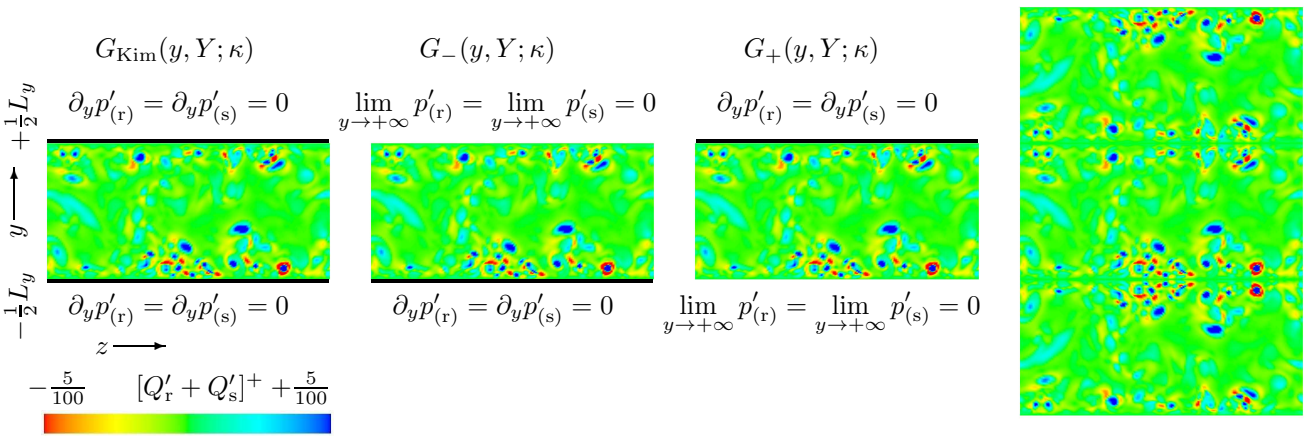

FiguRE 4. Instantaneous pressure fluctuation sources $\left(\operatorname{sum}\left[Q_{(\mathrm{r})}^{\prime}+Q_{(\mathrm{s})}^{\prime}\right]^{+}\right.$of rapid and slow sources in wall units) at $x=0$ (DNS; $R e_{\tau_{w}}=179 ; \bar{M}_{\mathrm{CL}}=0.34 ; 193 \times 129 \times 169$ grid; Tab. 1), and boundary conditions for the exact problem (2.6) whose solution (Kim 1989) is given by $\left.G_{\operatorname{Kim}}(y, Y ; \kappa) \sqrt{\mathrm{B} 10 b}\right)$, and for the two virtual halfspace problems with only one wall present defining $G_{ \pm}(y, \bar{Y} ; \kappa)(\mathrm{B} 18)$, which define the local wall-corrections appearing in the high-wavenumber approximation of the method of images (Manceau et al. 2001), corresponding to the approximate Green's function $G_{\mathrm{MWL}}(y, Y ; \kappa)(\mathrm{B} 42 c)$.

where $p_{(\mathrm{r} ; \mathfrak{V})}^{\prime}$ corresponds to the contribution of the volume integral in $\left.1.2 a\right), p_{(\mathrm{r} ; w)}^{\prime}$ corresponds to the contribution of the surface integral in $[1.2 a), p_{(\mathrm{s} ; \mathfrak{V})}^{\prime}$ corresponds to the contribution of the volume integral in $1.2 b$, and $p_{(\mathrm{s} ; w)}^{\prime}$ corresponds to the contribution of the surface integral in $1.2 b$.

Working directly with the surface integrals in 1.2 would have been a complex task, because they are implicit, ie they contain the value of the corresponding field at the wall. On the other hand, it is straightforward to evaluate the volume integrals in 1.2 , which contain only the sources and the 3-D freespace Green's function, which satisfies $\lim _{|\vec{x}-\vec{x}| \rightarrow \infty}|\overrightarrow{\mathfrak{x}}-\vec{x}|^{-1}=0$. Therefore the $x z$-Fourier-components of the volume terms should satisfy 2.6a), corresponding to the same distribution of sources as the complete physical problem, for $y \in\left[-\frac{1}{2} L_{y},+\frac{1}{2} L_{y}\right]$

$$
\left[\frac{\partial^{2}}{\partial y^{2}}-\kappa^{2}\right]\left[\begin{array}{c}
\hat{p}_{(\mathrm{r} ; \mathfrak{V})}^{\prime} \\
\hat{p}_{(\mathrm{s} ; \mathfrak{V})}^{\prime}
\end{array}\right]\left(\kappa_{x}, y, \kappa_{z}, t\right)=\left[\begin{array}{c}
\hat{Q}_{(\mathrm{r})}^{\prime} \\
\hat{Q}_{(\mathrm{s})}^{\prime}
\end{array}\right]\left(\kappa_{x}, y, \kappa_{z}, t\right)
$$

but not the homogeneous Neumann wall-boundary-conditions 2.6b), which are directly related to the surface integrals in 1.2 . They are required instead to decay as $|y| \rightarrow \infty$,

$$
\lim _{|y| \rightarrow \infty}\left[\begin{array}{c}
\hat{p}_{(\mathrm{r} ; \mathfrak{V})}^{\prime} \\
\hat{p}_{(\mathrm{s} ; \mathfrak{V})}^{\prime}
\end{array}\right]\left(\kappa_{x}, y, \kappa_{z}, t\right)=0
$$

ie they correspond to the solution of the hypothetical problem where $p^{\prime}$ is generated by the same distribution of sources in $y \in\left[-\frac{1}{2} L_{y},+\frac{1}{2} L_{y}\right]$ with the walls absent. Following the analysis in $\$$ B.2.3 for $\kappa \neq 0(\mathrm{~B} 14)$ and in $\$ \mathrm{~B} .3 .3$ for $\kappa=0$ (B 34), the solution of 2.11 reads

$$
\left[\begin{array}{c}
\hat{p}_{(\mathrm{r} ; \mathfrak{V})}^{\prime} \\
\hat{p}_{(\mathrm{s} ; \mathfrak{V})}^{\prime}
\end{array}\right]\left(\kappa_{x}, y, \kappa_{z}, t\right)=\int_{-\frac{1}{2} L_{y}}^{+\frac{1}{2} L_{y}} G_{\mathfrak{V}(}(y, Y ; \kappa)\left[\begin{array}{c}
\hat{Q}_{(\mathrm{r})}^{\prime} \\
\hat{Q}_{(\mathrm{s})}^{\prime}
\end{array}\right]\left(\kappa_{x}, Y, \kappa_{z}, t\right) d Y
$$


where the freespace Green's function

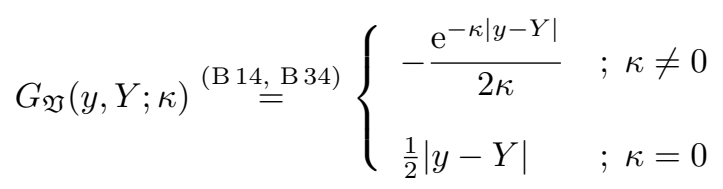

ensures boundedness at infinity. The same compatibility relation for the distribution of sources, as for the complete problem $(2.9 a)$, is required for the case $\kappa=0(\$ \mathrm{~B} .3 .3)$.

Because of the linearity of $1.1,1.2$, the splitting 2.10 readily implies

$$
\left[\begin{array}{l}
\hat{p}_{(\mathrm{r} ; w)}^{\prime} \\
\hat{p}_{(\mathrm{s} ; w)}^{\prime}
\end{array}\right]\left(\kappa_{x}, y, \kappa_{z}, t\right)=\left[\begin{array}{c}
\hat{p}_{(\mathrm{r})}^{\prime} \\
\hat{p}_{(\mathrm{s})}^{\prime}
\end{array}\right]\left(\kappa_{x}, y, \kappa_{z}, t\right)-\left[\begin{array}{c}
\hat{p}_{(\mathrm{r} ; \mathfrak{V})}^{\prime} \\
\hat{p}_{(\mathrm{s} ; \mathfrak{V})}^{\prime}
\end{array}\right]\left(\kappa_{x}, y, \kappa_{z}, t\right)
$$

Combining 2.7, 2.12, 2.13 we may define the wall-echo Green's function

$$
G_{w}(y, Y ; \kappa):=G_{\operatorname{Kim}}(y, Y ; \kappa)-G_{\mathfrak{V}}(y, Y ; \kappa)
$$

corresponding to the surface integrals (wall-echo) in $(1.2)$, which satisfies

$$
\left[\begin{array}{c}
\hat{p}_{(\mathrm{r} ; w)}^{\prime} \\
\hat{p}_{(\mathrm{s} ; w)}^{\prime}
\end{array}\right]\left(\kappa_{x}, y, \kappa_{z}, t\right)=\int_{-\frac{1}{2} L_{y}}^{+\frac{1}{2} L_{y}} G_{w}(y, Y ; \kappa)\left[\begin{array}{c}
\hat{Q}_{(\mathrm{r})}^{\prime} \\
\hat{Q}_{(\mathrm{s})}^{\prime}
\end{array}\right]\left(\kappa_{x}, Y, \kappa_{z}, t\right) d Y
$$

Notice that since, for $\kappa=0, G_{\text {Kim }}(y, Y ; \kappa=0)=G_{\mathfrak{V}}(y, Y ; \kappa=0)$ (B 35), we have $G_{w}(y, Y ; \kappa=0)=0$, ie wall-echo applies only on fields varying in at least one of the homogeneous directions $x$ or $z(\kappa \neq 0)$.

\subsubsection{The approximate method of images}

With the above developments we can directly evaluate the wall-echo terms for the slow and rapid parts in each of the correlations containing $p^{\prime}$ (\$3). By comparing with the exact solution obtained in the present work, it is possible to evaluate the approximation error of the method of images of Manceau et al. (2001).

The approximate method of images (Manceau et al. 2001) simply adds two mirror images of the channel, one above the upper wall and another below the lower wall (Fig. (4), to account for the presence of the walls, postulating that an approximation to the solution is obtained by using the freespace Green's function $G_{\mathfrak{V}}(y, Y ; \kappa)$ in the extended domain $y \in\left[-\frac{3}{2} L_{y},+\frac{3}{2} L_{y}\right]$. Taking into account the mirror symmetry of the ghost channels with respect to the corresponding wall (Fig. 4), this is equivalent ( B.4) to using an appropriate Green's function $G_{\mathrm{MWL}}(y, Y ; \kappa)$ B $\left.42 c\right)$ on the actual sources between the channel walls, $i e$ for $y \in\left[-\frac{1}{2} L_{y},+\frac{1}{2} L_{y}\right]$

$$
\left[\begin{array}{c}
\left.\hat{p}_{(\mathrm{r} ; \mathrm{MWL})}^{\prime}\right] \\
\left.\hat{p}_{(\mathrm{s} ; \mathrm{MWL})}^{\prime}\right]
\end{array}\right]\left(\kappa_{x}, y, \kappa_{z}, t\right)=\int_{-\frac{1}{2} L_{y}}^{+\frac{1}{2} L_{y}} G_{\mathrm{MWL}}(y, Y ; \kappa)\left[\begin{array}{c}
\hat{Q}_{(\mathrm{r})}^{\prime} \\
\hat{Q}_{(\mathrm{s})}^{\prime}
\end{array}\right]\left(\kappa_{x}, Y, \kappa_{z}, t\right) d Y
$$

By $(\mathrm{B} 42 c)$

$$
G_{\mathrm{MWL}}(y, Y ; \kappa)=G_{\mathfrak{V}}(y, Y ; \kappa)+G_{w_{-}}(y, Y ; \kappa)+G_{w_{+}}(y, Y ; \kappa)
$$

where $G_{w_{ \pm}}(y, Y ; \kappa)(\mathrm{B} 19)$ are the wall-echo Green's functions corresponding to the virtual halfspace problems with only one of the walls present (Fig. 4). Notice that the halfspace problems are solved exactly ( B.2.4,, B.3.4). Inherently, this is tantamount to assuming that there is no interaction between the echo effects of the upper and lower walls, since in the case of an isolated wall (halfspace problem; Fig. 4) the method of images yields the exact solution (Pope 2000, pp. 439-442). 

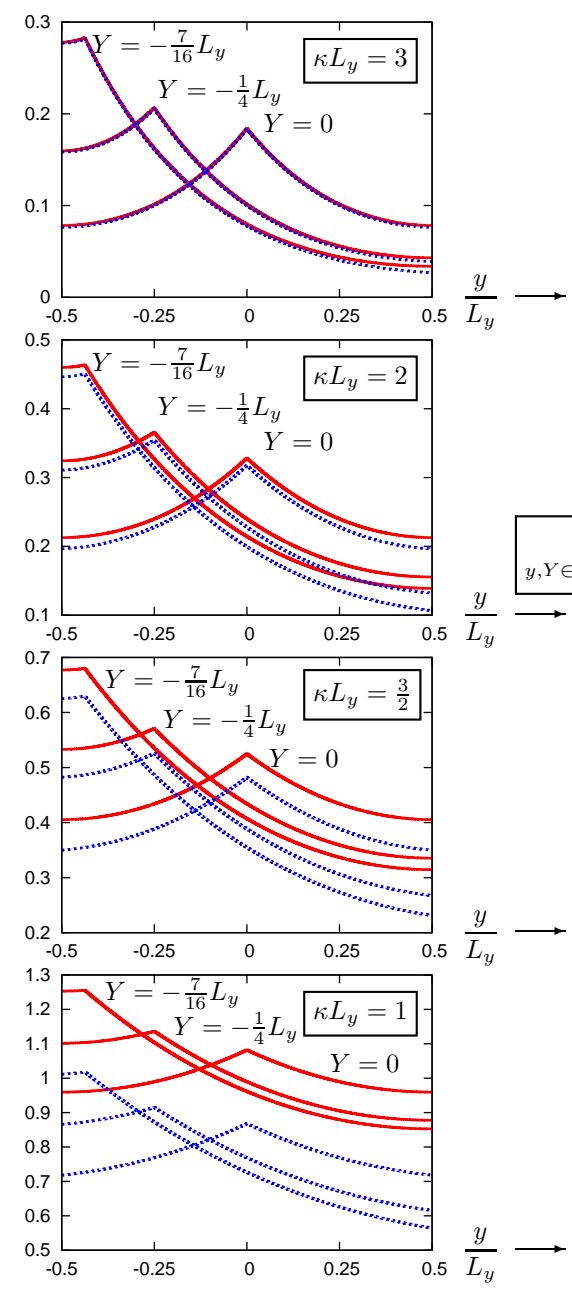

$$
\begin{aligned}
& -\frac{1}{L_{y}} G_{\mathrm{Kim}}(y, Y ; \kappa) \text { (exact) } \\
& \cdots \cdots . .-\frac{1}{L_{y}} G_{\mathrm{MWL}}(y, Y ; \kappa) \text { (approximate) }
\end{aligned}
$$
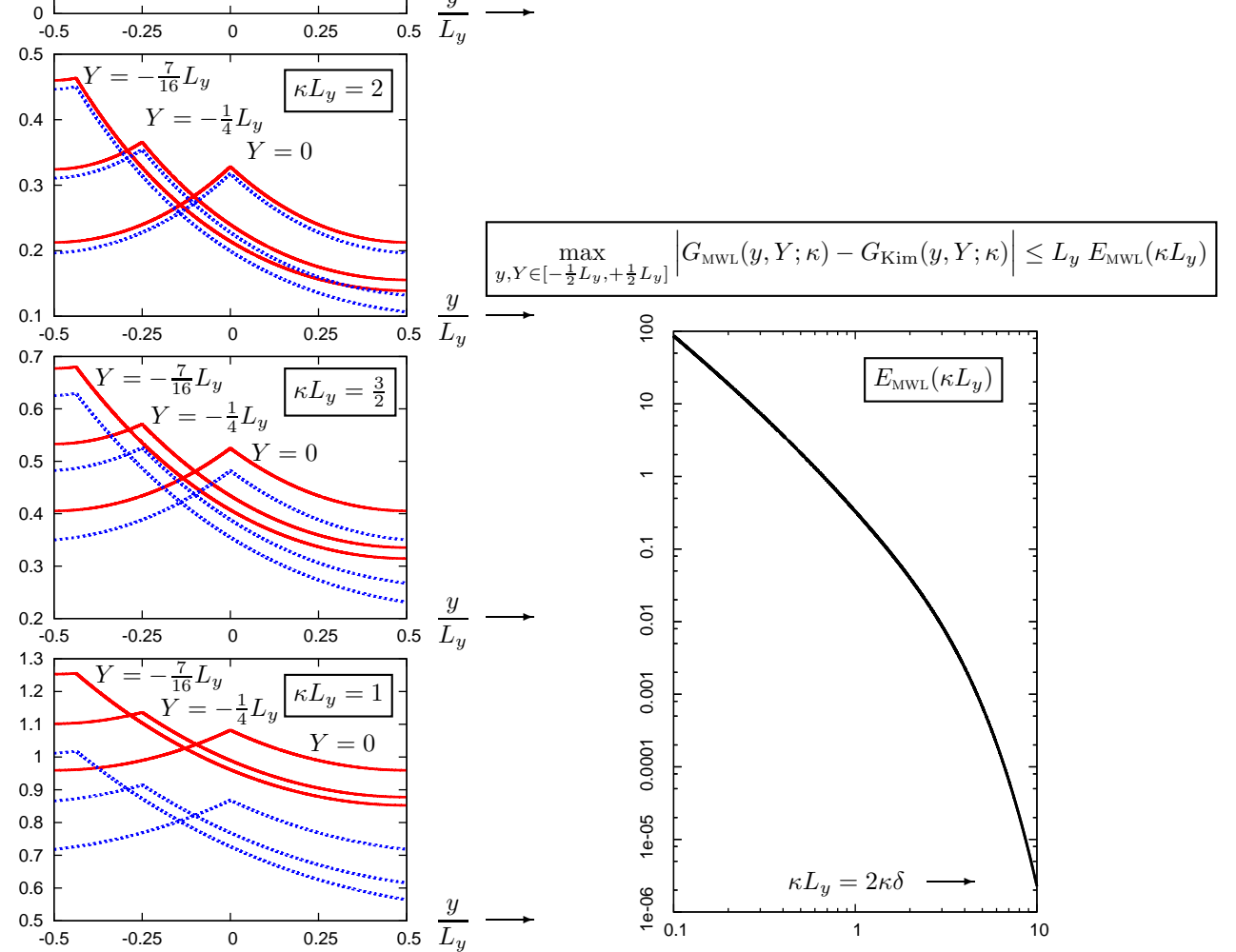

Figure 5. Comparison of the exact Green's function (B 10b), $G_{\text {Kim }}(y, Y ; \kappa)(\overline{\operatorname{Kim}} 1989)$, with the method-of-images approximation (B 42$), G_{\mathrm{MWL}}(y, Y ; \kappa)$ (Manceau et al. 2001), for $Y \in\left\{-\frac{7}{16} L_{y},-\frac{1}{4} L_{y}, 0\right\}$ and $\kappa L_{y} \in\left\{1, \frac{3}{2}, 2,3\right\}$, plotted as a function of $y L_{y}^{-1}$, and $\log$-plot of the upper bound estimate of the error made by the method-of-images approximation (B 45) as a function of the nondimensional wavenumber $\kappa L_{y}=2 \kappa \delta\left(L_{y}=2 \delta\right.$ is the channel height).

The approximation error of the method of images comes from the fact that the direct influence of the upper wall, approximated by the halfspace problem $G_{w_{+}}(y, Y ; \kappa)$ (Fig. 4), induces a nonzero gradient $\partial_{y}\left[\hat{p}_{(\mathrm{r} ;+)}^{\prime}, \hat{p}_{(\mathrm{s} ;+)}^{\prime}\right]^{\mathrm{T}}\left(\kappa_{x}, y=-\frac{1}{2} L_{y}, \kappa_{z}, t\right) \neq 0$ at the lower wall, and that there is no feedback from the lower wall to correct this (and vice versa). The nondimensional approximation error can be evaluated (\$B.4) by comparison with the exact solution (B 10b) of $\operatorname{Kim}(1989)$, and is a function of the nondimensional wavenumber $\kappa L_{y}=2 \kappa \delta(\mathrm{B} 45)$. For large nondimensional wavenumbers $\kappa L_{y}$, ie structures with small streamwise and spanwise extent compared to the channel height, this error is small, and rapidly decreases with increasing wavenumber (Fig. 5).

Let $\ell_{\kappa}:=2 \pi \kappa^{-1}$ be the representative size (wavelength) in the $x z$ plane of structures corresponding to $\kappa:=\sqrt{\kappa_{x}^{2}+\kappa_{z}^{2}} 22.6 c$. For $\kappa L_{y} \gtrsim 3 \Longleftrightarrow \ell_{\kappa} \lesssim 4 \delta$, the approximation of $G_{\mathrm{Kim}}(y, Y ; \kappa)(\mathrm{B} 10 b)$ by $G_{\mathrm{MWL}}(y, Y ; \kappa)(\mathrm{B} 42 c)$ is satisfactory (Fig. 5), the nondimen- 

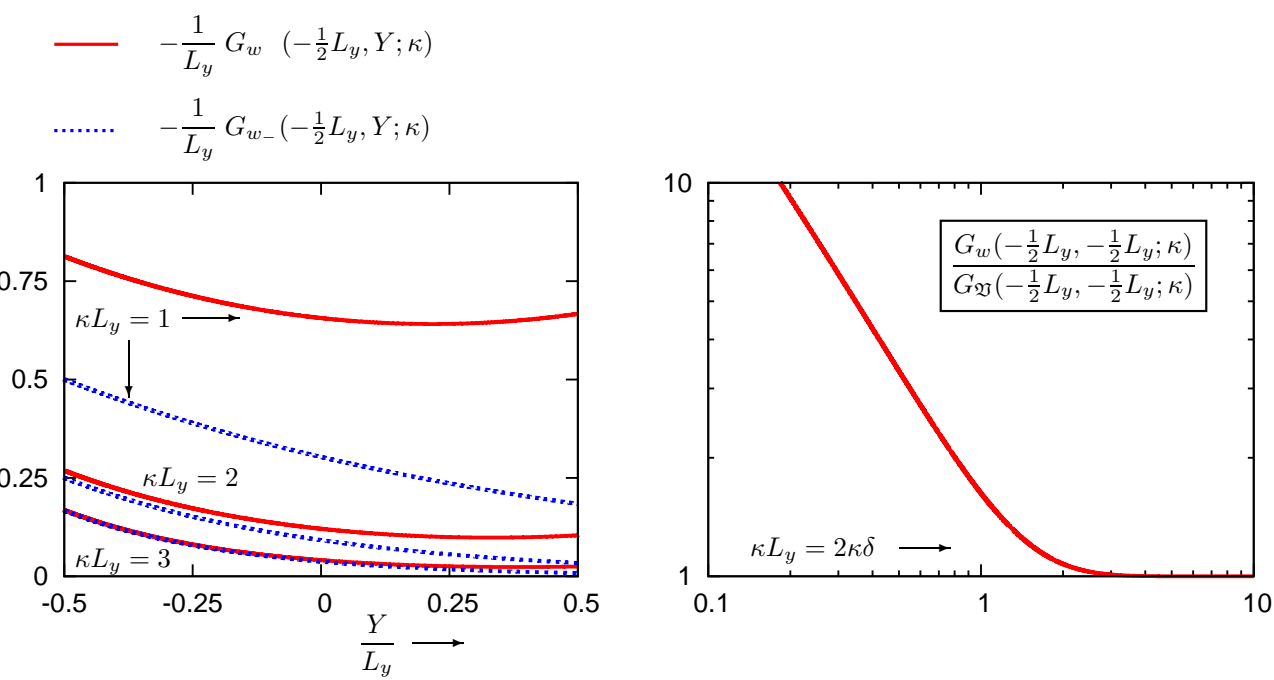

Figure 6. Comparison of the exact wall-echo Green's function $G_{w}\left(y=-\frac{1}{2} L_{y}, Y ; \kappa\right)$ (2.17), for the computation of the solution at the lower wall $y=-\frac{1}{2} L_{y}$, with the wall-echo Green's function $G_{w_{-}}\left(y=-\frac{1}{2} L_{y}, Y ; \kappa\right)(\mathrm{B} 42)$, of the corresponding halfspace problem with only the lower wall present (Fig. 44, for $\kappa L_{y} \in\{1,2,3\}$, plotted as a function of $Y L_{y}^{-1}$, and log-plot of the ratio at the wall $\left(y=Y=-\frac{1}{2} L_{y}\right)$ of the exact wall-echo Green's function $G_{w}\left(y=-\frac{1}{2} L_{y}, Y=-\frac{1}{2} L_{y} ; \kappa\right)$ 2.17 on the corresponding freespace Green's function $G_{\mathfrak{V}}\left(y=-\frac{1}{2} L_{y}, Y=-\frac{1}{2} L_{y} ; \kappa\right)$ (B 14) plotted against the nondimensional wavenumber $\kappa L_{y}=2 \kappa \delta\left(L_{y}=2 \delta\right.$ is the channel height).

sional error being $\lesssim 1 \%$. For the present case with $R e_{\tau_{w}} \approx 180$ this corresponds roughly to $\kappa^{+} \gtrsim \frac{1}{100}$, which includes the wavenumbers where most of the energy of the $p^{\prime}$-spectra is contained (Fig. 2), implying that the method of images is a satisfactory engineering approximation. However, for larger structures, eg $\kappa L_{y} \approx \frac{3}{2} \Longleftrightarrow \ell_{\kappa} \approx 8 \delta$, such as the superstructures observed in higher- $R e_{\tau_{w}}$ wall turbulence (Hutchins \& Marusic 2007, Balakumar \& Adrian 2007), the approximation error is roughly 10\%, and then grows exponentially with increasing size.

\subsubsection{Interaction of wall-echo between walls}

Since the freespace Green's function $G_{\mathfrak{V}}(y, Y ; \kappa)$ (B 14. B 35 is the same for all problems (Fig. 4), the differences (Fig. 5) between the exact solution $G_{\text {Kim }}(y, Y ; \kappa)(\mathrm{B} 10 b)$ and the method-of-images approximation $G_{\mathrm{MWL}}(y, Y ; \kappa)(\mathrm{B} 42)$, corresponds to increasingly strong interaction between the two walls with decreasing nondimensional wavenumber $\kappa L_{y}=2 \kappa \delta$, leading to amplification of the echo effect. To further explain this phenomenon notice the identity

$$
\begin{aligned}
G_{\mathfrak{V}}\left(y=-\frac{1}{2} L_{y}, Y ; \kappa \neq 0\right) & \stackrel{\frac{\mathrm{B} 14}{-}}{-}-\frac{\mathrm{e}^{\kappa\left(Y+\frac{1}{2} L_{y}\right)}}{2 \kappa} \\
& \stackrel{\mathrm{B} 18 a \mathrm{a}}{-} G_{-}\left(y=-\frac{1}{2} L_{y}, Y ; \kappa \neq 0\right)-G_{\mathfrak{V}}\left(y=-\frac{1}{2} L_{y}, Y ; \kappa \neq 0\right) \\
& \stackrel{\mathrm{B} 42 c]}{=} G_{w_{-}}\left(y=-\frac{1}{2} L_{y}, Y ; \kappa \neq 0\right) \quad \forall Y \in\left(-\frac{1}{2} L_{y},+\frac{1}{2} L_{y}\right)
\end{aligned}
$$



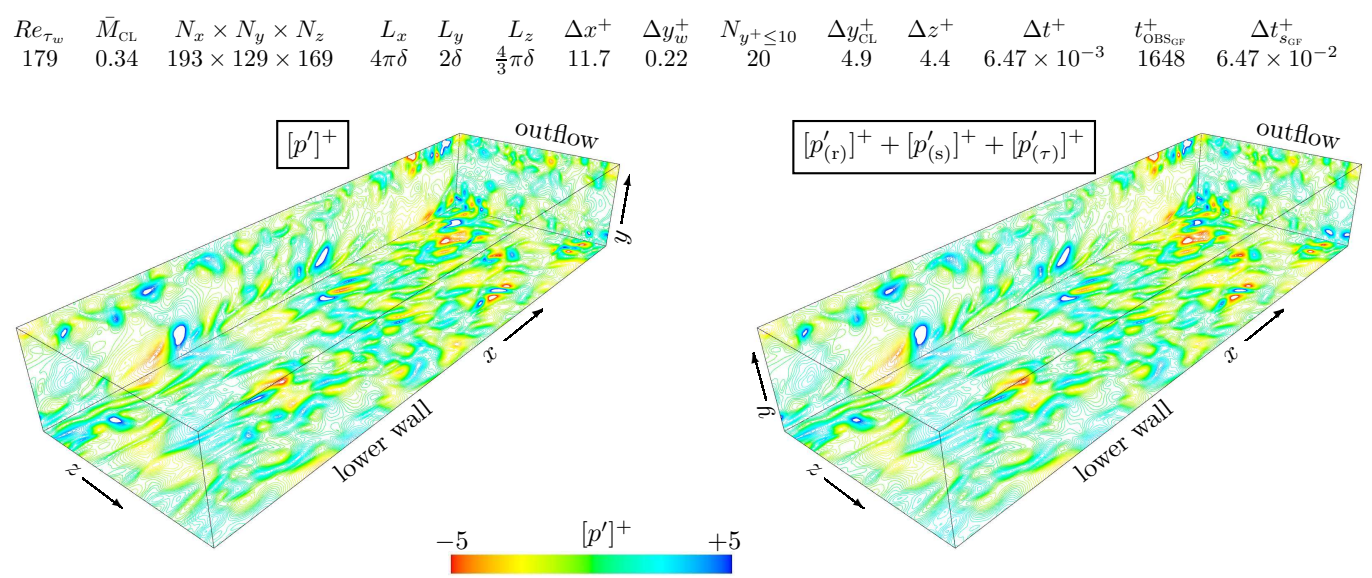

FIGURE 7. Instantaneous fluctuating pressure in wall units $\left[p^{\prime}\right]^{+}$obtained directly $\left(R e_{\tau_{w}}=179\right.$; $\bar{M}_{\mathrm{CL}}=0.34 ; 193 \times 129 \times 169$ grid; Tab. 1) by the compressible DNS solver (Gerolymos et al. $2010)$ compared to the superposition of the three fields, rapid $\left[p_{(\mathrm{r})}^{\prime}\right]^{+}$and slow $\left[p_{(\mathrm{s})}^{\prime}\right]^{+}$obtained by the Green's function solution 2.7 , and Stokes field $\left[p_{(\tau)}^{\prime}\right]^{+}(2.8)$, in wall units (50 contours in the range $[-5,+5]$ on the lower wall, the outflow $x$-periodic interface, and the $z$-periodicity interface).

which implies by (B 3) that for the halfspace problems (\$B.2.4) the wall-echo, at the wall $\left(y=-\frac{1}{2} L_{y}\right)$, exactly equals the volume term at the wall.

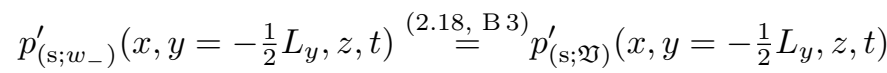

$$
\begin{aligned}
& p_{\left(\mathrm{r} ; w_{-}\right)}^{\prime}\left(x, y=-\frac{1}{2} L_{y}, z, t\right) \stackrel{2.18] \mathrm{B} 3}{-} p_{(\mathrm{r} ; \mathfrak{V})}^{\prime}\left(x, y=-\frac{1}{2} L_{y}, z, t\right)
\end{aligned}
$$

This result 2.19 following from the equality of the corresponding Green's functions (2.18) is valid independently of the particular sources $\hat{Q}(y ; \kappa)(2.6 a)$.

At high nondimensional wavenumbers $\kappa L_{y}$ the exact wall-echo Green's function $G_{w}(y=$ $\left.-\frac{1}{2} L_{y}, Y ; \kappa\right) 2$ 2.17), for the computation of $p_{w}^{\prime}$ at the lower wall $\left(y=-\frac{1}{2} L_{y}\right)$, is approximately equal $\left(\kappa L_{y}=3\right.$; Fig. 6 to the wall-echo Green's function $G_{w_{-}}\left(y=-\frac{1}{2} L_{y}, Y ; \kappa\right)$ (B 42) of the halfspace problem with only the lower wall present (Fig. 4), except at the upper part of the channel $Y \in\left[\frac{1}{4} L_{y}, \frac{1}{2} L_{y}\right]$, where the influence of the upper wall on the Green's function used for the computation of $p_{w}^{\prime}$ at the lower wall $\left(y=-\frac{1}{2} L_{y}\right)$ is felt. As the nondimensional wavenumber $\kappa L_{y}$ further decreases (ie the corresponding structure size $\ell_{\kappa} \delta^{-1}:=2 \pi \kappa^{-1} \delta^{-1}$ increases) the influence of the upper wall is felt further down (Fig. 4). Of course there is an analogous effect concerning the influence of the lower wall on the upper wall. As the nondimensional wavenumber $\kappa L_{y}$ decreases this mutual interaction between the two walls amplifies the echo effect, the amplification growing exponentially with structure size (Fig. 4).

\section{Analysis of pressure correlations}

The algorithm $(\$ 2.2 .3)$ for separating the rapid and slow contributions to $p^{\prime}(\$ 2.2 .2$ into weakly inhomogeneous volume $\left(p_{(\mathrm{r} ; \mathfrak{V})}^{\prime}\right.$ and $\left.p_{(\mathrm{s} ; \mathfrak{V})}^{\prime}\right)$ and strongly inhomogeneous wallecho terms $\left(p_{(\mathrm{r} ; w)}^{\prime}\right.$ and $\left.p_{(\mathrm{s} ; w)}^{\prime}\right)$, was applied to a low-Reynolds-number $\left(\operatorname{Re}_{\tau_{w}} \approx 180\right)$ well-resolved DNS (grid $193 \times 129 \times 169$; Tab. 1). The simulation was started at $t=t_{0}$ by interpolation of a well-converged simulation on a coarser grid (grid $129 \times 129 \times 129$; Tab. 1), and continued for $t_{\mathrm{GF}_{0}}^{+}-t_{0}^{+}=681$. During this interval $t^{+} \in\left[t_{0}^{+}, t_{\mathrm{GF}_{0}}^{+}\right]$statistics for 


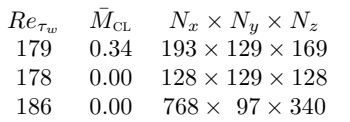

$\left[p^{\prime}\right]_{\mathrm{rms}}^{+}: \begin{cases}- & \text {present DNs } \\ \circ & \text { DNs Moser et al. (1999) } \\ \diamond & \text { DNs Hoyas and Jiménez }\end{cases}$
G.A. Gerolymos, D. Sénéchal and I. Vallet
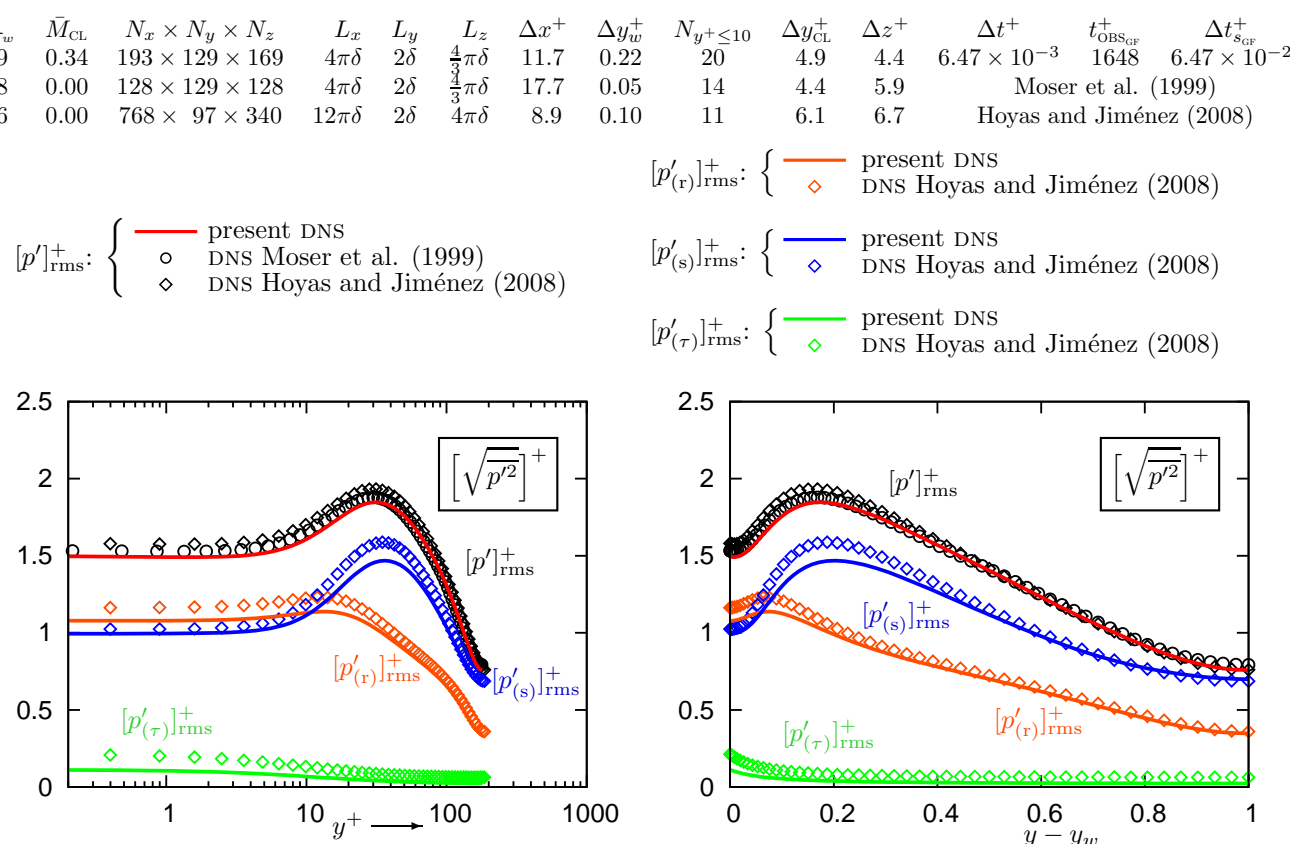

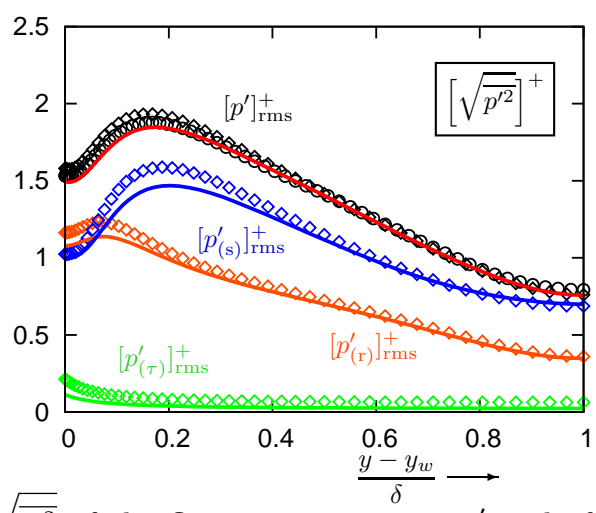

Figure 8. Comparison of rms-values $p_{\mathrm{rms}}^{\prime}:=\sqrt{\overline{p^{\prime 2}}}$ of the fluctuating pressure $p^{\prime}$ and of the fields $p_{(\mathrm{r})}^{\prime}+p_{(\mathrm{s})}^{\prime}+p_{(\tau)}^{\prime}=p^{\prime} 2.2$, from the present DNs computations $\left(R e_{\tau_{w}}=179 ; \bar{M}_{\mathrm{CL}}=0.34\right.$; $193 \times 129 \times 169$ grid; Tab. 1) with reference results of incompressible pseudospectral (Kim et al. 1987) DNS computations of Hoyas \& Jiménez $\left(2008, R e_{\tau_{w}}=186, M_{\mathrm{CL}}=0\right)$ and of Moser et al. (1999, $\left.R e_{\tau_{w}}=178, M_{\mathrm{CL}}=0\right)$, in wall units, plotted against the nondimensional distance-from-the-wall in inner $\left(y^{+}\right)$and outer $\left(\delta^{-1}\left(y-y_{w}\right)\right)$ scaling.

$\bar{u}_{i}$ and $\overline{u_{i}^{\prime} u_{j}^{\prime}}$, necessary for the computation of the source-terms in 2.3a, were computed with sampling at every iteration $\left(\Delta t_{s}^{+}=\Delta t^{+} \approx 6.47 \times 10^{-3} \Longleftrightarrow f_{s}^{+} \approx 154\right)$. Then the Green's function algorithm for $p^{\prime}$-splitting 2.10 was applied, and statistics of pressure correlations were computed for an observation time $t_{\mathrm{OBS}}^{+}=1648$, with sampling every 10 iterations $\left(\Delta t_{s_{\mathrm{GF}}}^{+}=10 \Delta t^{+} \approx 6.47 \times 10^{-2} \Longleftrightarrow f_{s_{\mathrm{GF}}}^{+} \approx 15.4\right)$.

\subsection{Fluctuating pressure field}

The instantaneous fluctuating pressure $p^{\prime}$-field reconstructed by the Green's function approach 2.7 2.8) according to the splitting (2.2) agrees quite well (Fig. 7) with the instantaneous $p^{\prime}$-field directly computed (without making use of Green's functions) by the compressible DNS solver (Gerolymos et al. 2010).

Both the directly computed $p_{\mathrm{rms}}^{\prime}$ and the rms values $\left(\left[p_{(\mathrm{r})}^{\prime}\right]_{\mathrm{rms}},\left[p_{(\mathrm{s})}^{\prime}\right]_{\mathrm{rms}},\left[p_{(\tau)}^{\prime}\right]_{\mathrm{rms}}\right)$ of the $p^{\prime}$-splitting (2.2) compare globally satisfactorily (Fig. 8) with standard results from incompressible DNS computations (Kim et al. 1987, Hoyas \& Jiménez 2008), both in the wall and the outer regions (Fig. 8). Computed $p_{\text {rms }}^{\prime}$ corresponds, for the present DNS results (Fig. 8), to the fluctuation of the actual thermodynamic pressure, obtained from the equation-of-state (Gerolymos et al. 2010$)$. There is quite good agreement of present results for $p_{\text {rms }}^{\prime}$ with the incompressible DNS of Kim et al. (1987), obtained with similar resolution on the same computational box (Fig. 8), the present data being marginally lower, because of the small mean-density-variation effect $(\lesssim 1.5 \%$; 2.1 . The incompressible DNS data of Hoyas \& Jiménez (2008), with similar resolution but a larger computational box indicate slightly higher $\left[p^{\prime}\right]_{\mathrm{rms}}^{+}$(Fig. 8). This is attributed to the higher 

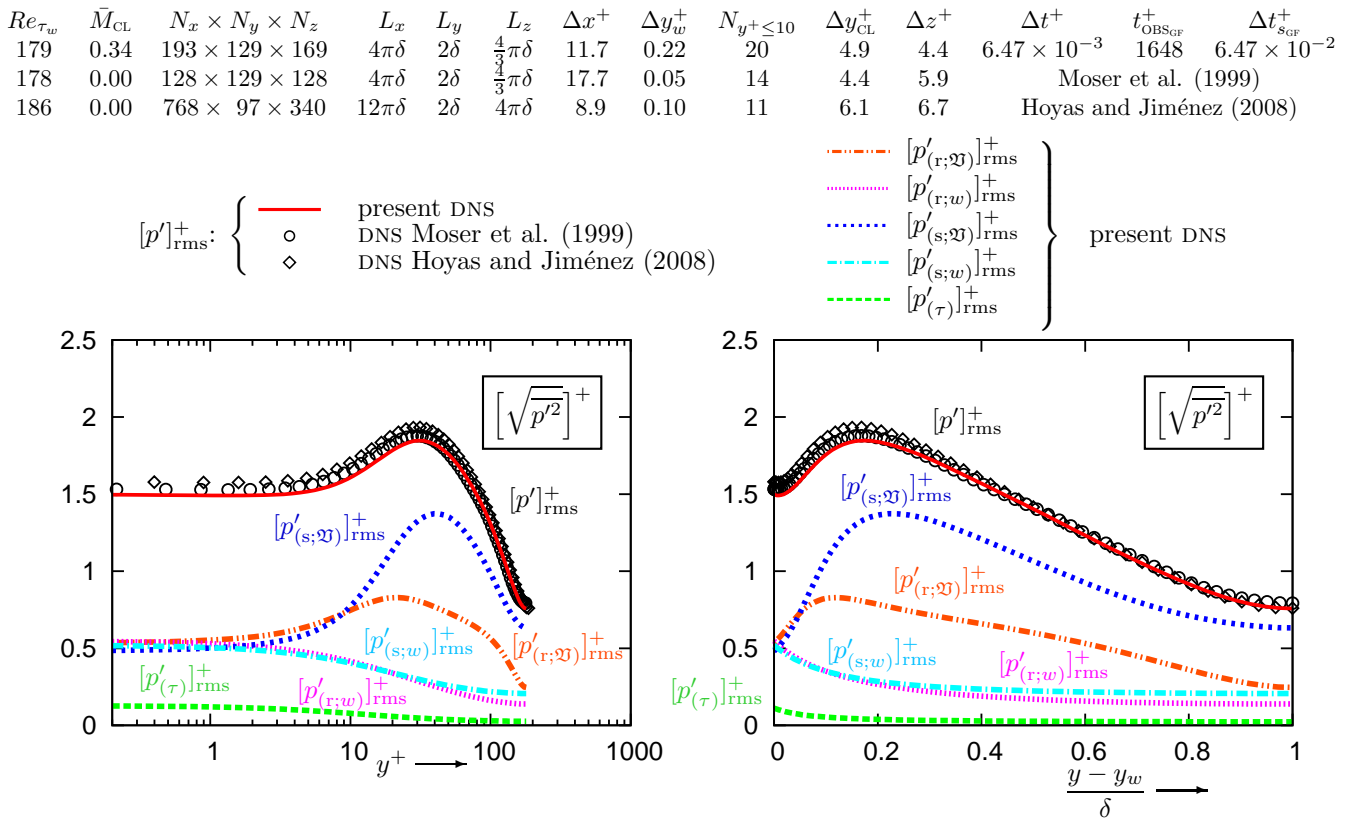

Figure 9. Rms-values of the 5 fluctuating pressure fields in the decomposition $p^{\prime}=p_{(\mathrm{r} ; \mathfrak{V})}^{\prime}+p_{(\mathrm{r} ; w)}^{\prime}+p_{(\mathrm{s} ; \mathfrak{V})}^{\prime}+p_{(\mathrm{s} ; w)}^{\prime}+p_{(\tau)}^{\prime} 2.10$, from the present DNS computations $\left(R e_{\tau_{w}}=179 ; \bar{M}_{\mathrm{CL}}=0.34 ; 193 \times 129 \times 169\right.$ grid; Tab. 1), plotted against the nondimensional distance-from-the-wall in inner $\left(y^{+}\right)$and outer $\left(\delta^{-1}\left(y-y_{w}\right)\right)$ scaling, and $p_{\mathrm{rms}}^{\prime}$ from various DNS databases (Moser et al. 1999 , Hoyas \& Jiménez 2008; Gerolymos et al. 2010).

$R e_{\tau_{w}}=186$ in the simulations of Hoyas \& Jiménez (2008), compared to $R e_{\tau_{w}}=178$ Kim et al. (1987) and $R e_{\tau_{w}}=179$ for the present simulations. The variation of $\left[p_{w}^{\prime}\right]_{\mathrm{rms}}^{+}$ with $\operatorname{Re}_{\tau_{w}}$ (Hu et al. 2006 ; Tsuji et al. 2007 ), indicates a $\sim 2 \%$ increase in $\left[p_{w}^{\prime}\right]_{\mathrm{rms}}^{+}$from $R e_{\tau_{w}}=178$ to $R e_{\tau_{w}}=186$. Incompressible DNs data of the different fields $\left(\left[p_{(\mathrm{r})}^{\prime}\right]_{\mathrm{rms}}\right.$, $\left.\left[p_{(\mathrm{s})}^{\prime}\right]_{\mathrm{rms}},\left[p_{(\tau)}^{\prime}\right]_{\mathrm{rms}}\right)$ were available only for the simulation of Hoyas \& Jiménez (2008), and are expectedly a little higher than those of the present computations (Fig. 8), consistently with the difference in $\left[p^{\prime}\right]_{\mathrm{rms}}^{+}$用 There is, nonetheless, a difference in the level of Stokes pressure $\left[p_{(\tau)}^{\prime}\right]_{\mathrm{rms}}$ near the wall (Fig. 8), which should be further investigated, but this term is quite small compared to the others.

The new results (Fig. $9 p$ in the present work concern the further splitting $\left(p^{\prime}=p_{(\mathrm{r} ; \mathfrak{V})}^{\prime}+\right.$ $\left.p_{(\mathrm{r} ; w)}^{\prime}+p_{(\mathrm{s} ; \mathfrak{V})}^{\prime}+p_{(\mathrm{s} ; w)}^{\prime}+p_{(\tau)}^{\prime}\right)$ into volume and wall-echo terms 2.10. The rms-levels of wall-echo $\left(\left[p_{(\mathrm{r} ; w)}^{\prime}\right]_{\mathrm{rms}},\left[p_{(\mathrm{s} ; w)}^{\prime}\right]_{\mathrm{rms}}\right)$, at the wall $(y=0$; Fig. 9 , are approximately equal to the corresponding volume terms $\left(\left[p_{(\mathrm{r} ; \mathfrak{V})}^{\prime}\right]_{\mathrm{rms}},\left[p_{(\mathrm{s} ; \mathfrak{V})}^{\prime}\right]_{\mathrm{rms}}\right)$. This implies $(2.2 .5$ that the dominant contributions to the spectra $2.5 b$ of the source-terms $\hat{Q}_{(\mathrm{r})}^{\prime}\left(\kappa_{x}, y, \kappa_{z}, t\right)$ and $\hat{Q}_{(\mathrm{s})}^{\prime}\left(\kappa_{x}, y, \kappa_{z}, t\right) 2.6 a$ occur at sufficiently high nondimensional wavenumbers $\kappa L_{y}=2 \kappa \delta$ (Fig. 6), for the interaction between the wall-echo from the upper wall and the wall-echo from the lower wall to be negligibly small. This approximate equality of wall-echo and volume terms holds up to $y^{+} \lesssim 2$ (Fig. 9). Further away from the wall, both wallecho terms $\left(\left[p_{(\mathrm{r} ; w)}^{\prime}\right]_{\mathrm{rms}},\left[p_{(\mathrm{s} ; w)}^{\prime}\right]_{\mathrm{rms}}\right)$ decay with increasing distance from the wall, always remaining much higher than the Stokes pressure $\left[p_{(\tau)}^{\prime}\right]_{\mathrm{rms}}$ (Fig. 9p. In the buffer and

$\dagger$ Notice also that $\overline{p^{\prime 2}}=\overline{\left(p_{(\mathrm{r})}^{\prime}+p_{(\mathrm{s})}^{\prime}+p_{(\tau)}^{\prime}\right)^{2}} \neq \overline{p_{(\mathrm{r})}^{\prime 2}}+\overline{p_{(\mathrm{s})}^{\prime 2}}+\overline{p_{(\tau)}^{\prime 2}}$ 
$\begin{array}{ccccccccccccccc}R e_{\tau_{w}} & \bar{M}_{\mathrm{CL}} & N_{x} \times N_{y} \times N_{z} & L_{x} & L_{y} & L_{z} & \Delta x^{+} & \Delta y_{w}^{+} & N_{y^{+} \leq 10} & \Delta y_{\mathrm{CL}}^{+} & \Delta z^{+} & \Delta t^{+} & t_{\mathrm{OBS}}^{+} & \Delta t_{s_{\mathrm{GF}}}^{+} \\ 179 & 0.34 & 193 \times 129 \times 169 & 4 \pi \delta & 2 \delta & \frac{4}{3} \pi \delta & 11.7 & 0.22 & 20 & 4.9 & 4.4 & 6.47 \times 10^{-3} & 1648 & 6.47 \times 10^{-2}\end{array}$

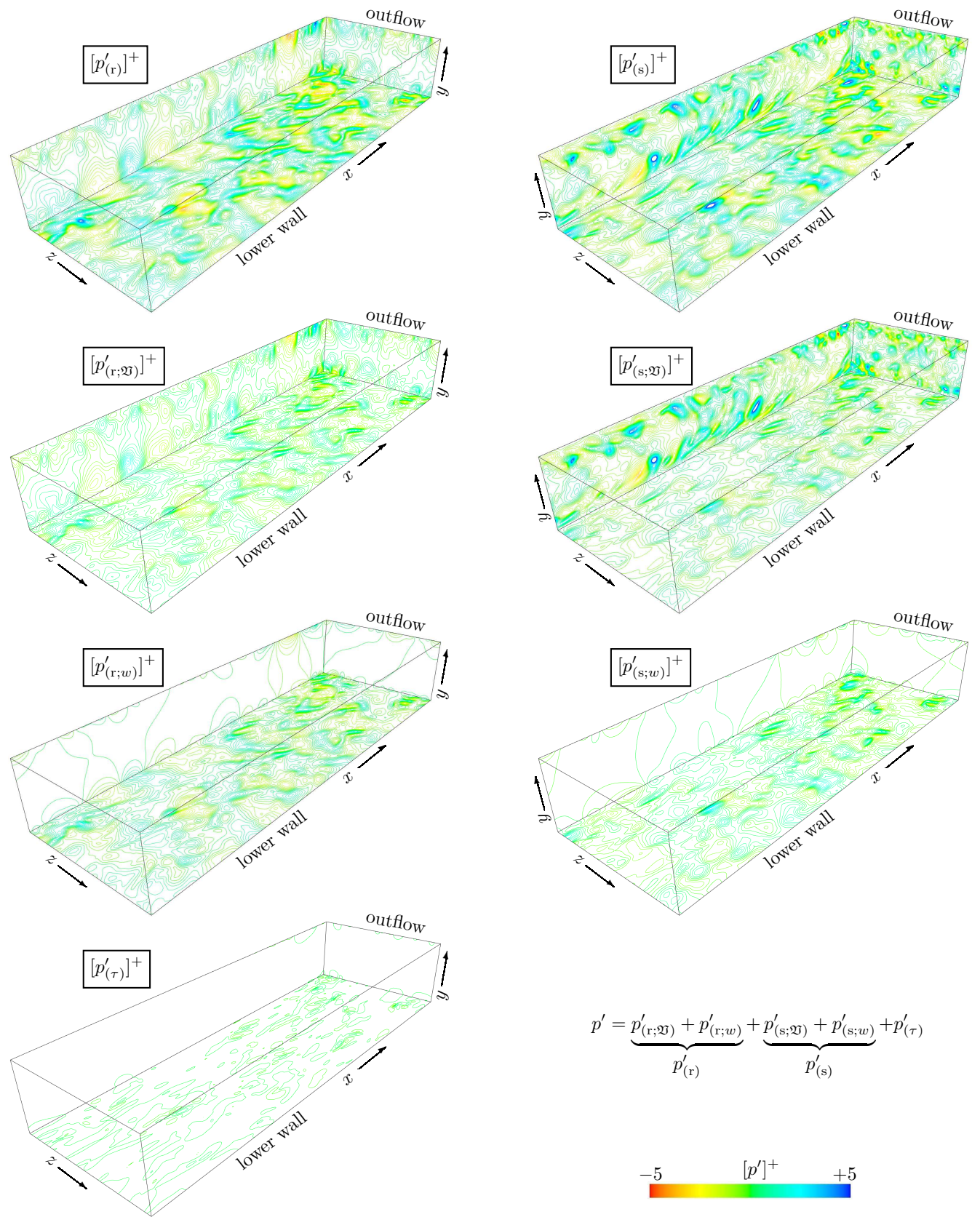

FIGURE 10. Instantaneous fluctuating pressure fields 2.10 from DNS computations $\left(R e_{\tau_{w}}=179\right.$; $\bar{M}_{\mathrm{CL}}=0.34 ; 193 \times 129 \times 169$ grid; Tab. 1), Stokes $\left[p_{(\tau)}^{\prime}\right]^{+}(2.8)$, and rapid $\left[p_{(\mathrm{r})}^{\prime}\right]^{+}$and slow $\left[p_{(\mathrm{s})}^{\prime}\right]^{+}$ 2.7 , and their decomposition into weakly inhomogeneous volume fields, $\left[p_{(\mathrm{r} ; \mathfrak{V})}^{\prime}\right]^{+}$and $\left[p_{(\mathrm{s} ; \mathfrak{V})}^{\prime}\right]^{+}$ 2.12, and strongly inhomogeneous wall-echo fields, $\left[p_{(\mathrm{r} ; w)}^{\prime}\right]^{+}$and $\left[p_{(\mathrm{s} ; w)}^{\prime}\right]^{+}[2.15$, in wall units (50 contours in the range $[-5,+5]$ on the lower wall, the outflow $x$-periodic interface, and the $z$-periodicity interface). 
outer regions $\left(y-y_{w} \gtrsim \frac{5}{100} \delta \stackrel{R e_{\tau_{w}} \approx}{\rightleftharpoons} 180 y^{+} \gtrsim 10\right)$ the well known predominance of the slow volume term $\left[p_{(\mathrm{s} ; \mathfrak{V})}^{\prime}\right]_{\mathrm{rms}}$ over the rapid volume term $\left[p_{(\mathrm{r} ; \mathfrak{V})}^{\prime}\right]_{\mathrm{rms}}(\operatorname{Kim} 1989$; Chang et al. 1999) is evident (Fig. 9). Nonetheless, it is essential, from the point-of-view of near-wall modelling, to notice that for $y^{+} \lesssim 10,\left[p_{(\mathrm{r} ; \mathfrak{V})}^{\prime}\right]_{\mathrm{rms}} \approx\left[p_{(\mathrm{s} ; \mathfrak{V})}^{\prime}\right]_{\mathrm{rms}} \approx\left[p_{(\mathrm{r} ; w)}^{\prime}\right]_{\mathrm{rms}} \approx$ $\left[p_{(\mathrm{s} ; w)}^{\prime}\right]_{\mathrm{rms}}>\left[p_{(\tau)}^{\prime}\right]_{\mathrm{rms}}$ (Fig. 9p. Since all of the terms are of the same order-of-magnitude near the wall, but may have different phases, and hence different correlation-coefficients with the fluctuating velocity field, they all require accurate modelling. Despite the fact that further away from the wall $\left(y^{+} \gtrsim 50\right)$ homogeneous models are reasonably accurate (Gerolymos et al. 2012a , Fig. 2, p. 9), global predictive performance of models in actual flows is dominated by the quality of near-wall modelling (Durbin 1993: Hanjalić 1994).

The above quantitative results (Fig. 9 ) are also observed qualitatively in the instantaneous levels (Fig. 10) of the 5 terms in the $p^{\prime}$-splitting (2.10). Comparison of instantaneous levels of the slow $\left(p_{(\mathrm{s})}^{\prime}\right)$ and rapid $\left(p_{(\mathrm{r})}^{\prime}\right)$ terms (Fig. 10 clearly shows that both mechanisms of generation of $p^{\prime} 2.3 a$ are of the same magnitude at the wall (lower wall; Fig. 10), while $p_{(\mathrm{s})}^{\prime}$ is the main mechanism (Chang et al. 1999) further away from the wall $(z=$ const plane; Fig. 10$)$. The same observations apply to the corresponding volume terms, $p_{(\mathbf{r} ; \mathfrak{V})}^{\prime}$ and $p_{(\mathrm{s} ; \mathfrak{V})}^{\prime}$ (Fig. 10p. The wall-echo terms, $p_{(\mathrm{r} ; w)}^{\prime}$ and $p_{(\mathbf{s} ; w)}^{\prime}$, at the wall (lower wall; Fig. 10), are approximately equal to the corresponding volume terms, $p_{(\mathrm{r} ; \mathfrak{V})}^{\prime}$ and $p_{(\mathrm{s} ; \mathfrak{V})}^{\prime}$, but rapidly decay away from the wall $(z=$ const plane; Fig. 10 . Finally, the Stokes pressure $p_{(\tau)}^{\prime}$ is substantially lower than the other terms and rapidly decays away from the wall (Fig. 10).

The results (Figs. 7 10 on the $p^{\prime}$-fields 2.10 provide guidance on the relative importance of each of the $5 p^{\prime}$-fields. Nonetheless, the transposition of these results to the decomposition of correlations containing $p^{\prime}$ is not always straightforward, especially in the near-wall region $\left(y^{+} \lesssim 10\right)$. For this reason we study in detail $(3.3$, 3.4 , 3.5 the correlations containing $p^{\prime}$ which appear in the transport equations for the Reynolds-stresses $(\$ 3.2$.

\subsection{Reynolds-stress transport}

The equations governing the Reynolds-stress tensor are central in single-point closure turbulence modelling (Lumley 1978; Hanjalić 1994). The exact transport equations for the Reynolds-stresses in incompressible flow read (Pope 2000, pp. 315-320)

$$
\begin{aligned}
& \underbrace{\frac{\partial \rho \overline{u_{i}^{\prime} u_{j}^{\prime}}}{\partial t}+\frac{\partial}{\partial x_{\ell}}\left(\rho \overline{u_{i}^{\prime} u_{j}^{\prime}} \bar{u}_{\ell}\right)}_{\text {convection } C_{i j}}=\underbrace{\frac{\partial}{\partial x_{\ell}}\left(-\rho \overline{u_{i}^{\prime} u_{j}^{\prime} u_{\ell}^{\prime}}-\overline{p^{\prime} u_{j}^{\prime}} \delta_{i \ell}-\overline{p^{\prime} u_{i}^{\prime}} \delta_{j \ell}+\mu \frac{\partial \overline{u_{i}^{\prime} u_{j}^{\prime}}}{\partial x_{\ell}}\right)}_{\text {diffusion } d_{i j}^{(u)}+d_{i j}^{(p)}+d_{i j}^{(\mu)}} \\
& +\underbrace{p^{\prime}\left(\frac{\partial u_{i}^{\prime}}{\partial x_{j}}+\frac{\partial u_{j}^{\prime}}{\partial x_{i}}\right)}_{\text {redistribution } \phi_{i j}}+\underbrace{\left(-\rho \overline{u_{i}^{\prime} u_{\ell}^{\prime}} \frac{\partial \tilde{u}_{j}}{\partial x_{\ell}}-\rho \overline{u_{j}^{\prime} u_{\ell}^{\prime}} \frac{\partial \tilde{u}_{i}}{\partial x_{\ell}}\right)}_{\text {production } P_{i j}} \\
& -\underbrace{\left.\left(\frac{\partial}{\partial x_{\ell}} \overline{\left(\mu \frac{\partial u_{i}^{\prime} u_{j}^{\prime}}{\partial x_{\ell}}\right.}\right)-\overline{\left(u_{i}^{\prime} \frac{\partial \tau_{j \ell}^{\prime}}{\partial x_{\ell}}+u_{j}^{\prime} \frac{\partial \tau_{i \ell}^{\prime}}{\partial x_{\ell}}\right)}\right)} \\
& \text { dissipation } \bar{\rho} \varepsilon_{i j}^{(\mu)}:=2 \mu \overline{\partial_{x_{\ell}} u_{i}^{\prime} \partial_{x_{\ell}} u_{j}^{\prime}}
\end{aligned}
$$

Convection $C_{i j}$, production $P_{i j}$ and viscous diffusion $d_{i j}^{(\mu)}$ are exact terms, while all the other terms $\left(d_{i j}^{(u)}, d_{i j}^{(p)}, \phi_{i j}\right.$, and $\left.\varepsilon_{i j}^{(\mu)}\right)$ require modelling. Pressure-diffusion $d_{i j}^{(p)}$ and 
redistribution $\phi_{i j}$ can be grouped together into the velocity/pressure-gradient correlation tensor $\Pi_{i j}$

$$
\Pi_{i j}:=-\overline{u_{i}^{\prime} \frac{\partial p^{\prime}}{\partial x_{j}}}-\overline{u_{j}^{\prime} \frac{\partial p^{\prime}}{\partial x_{i}}}=\underbrace{\overline{p^{\prime}\left(\frac{\partial u_{i}^{\prime}}{\partial x_{j}}+\frac{\partial u_{j}^{\prime}}{\partial x_{i}}\right)}}_{\overline{2 p^{\prime} S_{i j}^{\prime}}}-\underbrace{\left(\overline{\left.\frac{\partial p^{\prime} u_{i}^{\prime}}{\partial x_{j}}+\frac{\partial p^{\prime} u_{j}^{\prime}}{\partial x_{i}}\right)}\right.}_{d_{i j}^{(p)}} \stackrel{3.1 p}{=} \phi_{i j}+d_{i j}^{(p)}
$$

The velocity/pressure-gradient tensor $\Pi_{i j}$, which is the term appearing in most of the original early developments (Chou 1945), tends to 0 as $y \rightarrow 0$ (in the viscous sublayer, where $d_{i j}^{(p)}$ and $\phi_{i j}$ cancel one another). In homogeneous turbulence (HT) $d_{i j}^{(p)}=0 \stackrel{\mathrm{HT}}{\Longleftrightarrow}$ $\phi_{i j}=\Pi_{i j}$.

\subsection{Pressure transport $\overline{p^{\prime} u_{i}^{\prime}}$ and pressure diffusion $d_{i j}^{(p)}$}

The $p^{\prime}$-splitting 2.10 applied to pressure transport $\overline{p^{\prime} u_{i}^{\prime}}$ and pressure diffusion $d_{i j}^{(p)} 3.1$. 3.2 indicates (Fig. 11) that the slow volume term $\overline{p_{(\mathrm{s} ; \mathfrak{V})}^{\prime} v^{\prime}}$ is the principal contribution to the normal-to-the-wall transport $\overline{p^{\prime} v^{\prime}}$, and evenmore to $d_{y y}^{(p)}$ (for fully developed plane channel flow $\left.d_{y y}^{(p)} 3.13 .2-2 \partial_{y} \overline{p^{\prime} v^{\prime}}\right)$, except in the near-wall region $\left(y^{+} \lesssim 5\right)$ where all terms are of comparable importance (Fig. 11). Concerning $d_{x y}^{(p)}$ (for fully developed plane channel flow $\left.\left.d_{x y}^{(p)} 3.13 .2\right]-\partial_{y} \overline{p^{\prime} u^{\prime}}\right)$, again the slow volume term $d_{x y}^{(p ; s ; \mathfrak{T})}$ is the most important contribution in the buffer and outer regions, whereas in the near-wall region $\left(y^{+} \lesssim 5\right)$ all terms are of comparable importance (Fig. 11 .

For modelling purposes it is important to notice that, although the slow volume term $d_{i j}^{(p ; s ; \mathfrak{V})}$ is the main contribution (and a reasonable approximation) to $d_{i j}^{(p)}$ (Fig. 11, in the buffer and outer regions $\left(y^{+} \gtrsim 5\right)$, this is not the case for pressure transport $p^{\prime} u_{i}^{\prime}$, especially for $\overline{p^{\prime} u^{\prime}}$ (Fig. 11 , where, for $5 \lesssim y^{+} \lesssim 30, \overline{p_{(\mathrm{s} ; \mathfrak{V})}^{\prime} u^{\prime}}$ is of the opposite sign with respect to $\overline{p^{\prime} u^{\prime}}$.

These remarks imply that a consistent model for pressure diffusion $d_{i j}^{(p)}$, in the buffer and outer regions $\left(y^{+} \gtrsim 5\right)$, can be built using only slow volume terms. However, such a model for $d_{i j}^{(p)}\left(y^{+} \gtrsim 5\right)$, based on $d_{i j}^{(p ; ; ; \mathfrak{V})}$ only, cannot correspond to (or be built from) a satisfactory model for pressure transport $\overline{p^{\prime} u_{i}^{\prime}}$ (Fig. 11). The near-wall modelling of pressure diffusion $d_{i j}^{(p)}\left(y^{+} \lesssim 5\right)$ is more complex, since (Fig. 11$)$ it must contain rapid terms and wall-echo terms (Fig. 11). To the authors' knowledge (Gerolymos et al.|2012b) no single-point closure for $d_{i j}^{(p)}$ takes into account the wall-echo terms (Donaldson 1969 Hirt 1969, Daly \& Harlow 1970, Lumley 1978, Fu 1993, Sauret \& Vallet 2007; Vallet 2007).

\subsection{Pressure-strain redistribution $\phi_{i j}$}

The application of the $p^{\prime}$-splitting $(2.10)$ to pressure-strain redistribution $\phi_{i j}$ (3.1, 3.2) shows again that the volume terms $\left(\phi_{i j}^{(\text {r: } \mathfrak{V})}\right.$ and $\left.\phi_{i j}^{(\mathrm{s} ; \mathfrak{V})}\right)$ are the dominant contribution to $\phi_{i j}$ in the buffer and outer regions $\left(y^{+} \gtrsim 10\right.$; Fig. 12 . Notice that $\phi_{z z}^{(\mathrm{r} ; \mathfrak{V})}>\phi_{z z}^{(\mathrm{s} ; \mathfrak{V})}$ for $y^{+} \gtrsim 10$ (Fig. 12 , contrary to the other components for which the slow volume terms are the more important contributions to $\phi_{i j}$ in the buffer and outer regions $\left(y^{+} \gtrsim 15\right.$; Fig. 12).

In the near-wall region $\left(y^{+} \lesssim 10\right)$ the wall-echo terms $\left(\phi_{i j}^{(\mathrm{r} ; w)}\right.$ and $\left.\phi_{i j}^{(\mathrm{s} ; w)}\right)$ are of the same order-of-magnitude as the corresponding volume terms $\left(\phi_{i j}^{(\mathrm{r} ; \mathfrak{V})}\right.$ and $\phi_{i j}^{(\mathrm{s} ; \mathfrak{V})}$, respec- 

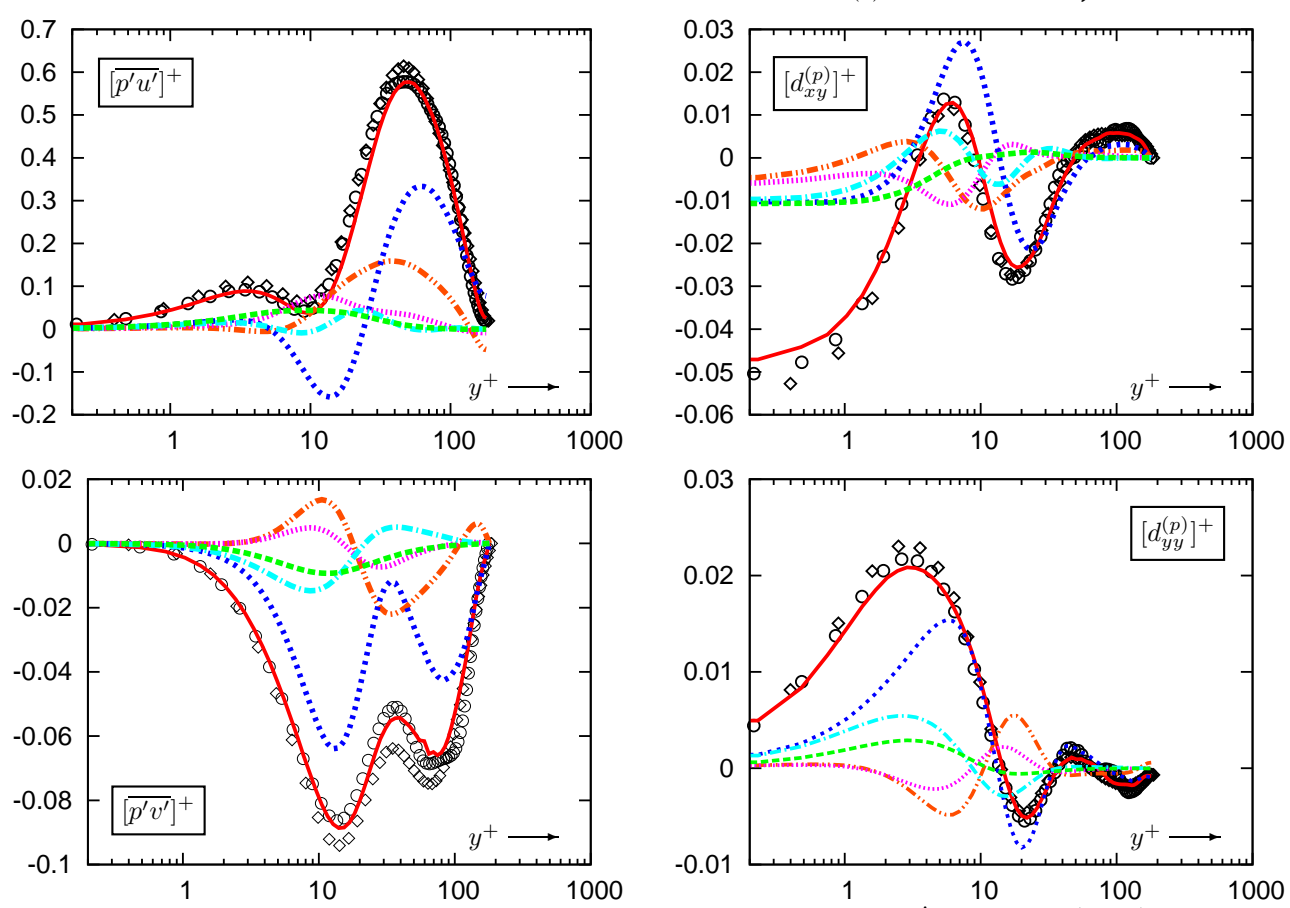

FIgURE 11. Distributions of the 5 terms in the incompressible $p^{\prime}$-splitting 2.10 of pressure transport, $\overline{p_{(\mathrm{r} ; \mathfrak{V})}^{\prime} u_{i}^{\prime}}, \overline{p_{(\mathrm{r} ; w)}^{\prime} u_{i}^{\prime}}, \overline{p_{(\mathrm{s} ; \mathfrak{V})}^{\prime} u_{i}^{\prime}}, \overline{p_{(\mathrm{s} ; w)}^{\prime} u_{i}^{\prime}}$, and $\overline{p_{(\tau)}^{\prime} u_{i}^{\prime}}$, from the present DNS computations $\left(R e_{\tau_{w}}=179 ; \bar{M}_{\mathrm{CL}}=0.34 ; 193 \times 129 \times 169\right.$ grid; Tab. 1), distributions of pressure transport $\overline{p^{\prime} u_{i}^{\prime}}$ from various DNS databases (Moser et al. 1999. Hoyas \& Jiménez 2008, Gerolymos et al. 2010 ), and corresponding contributions to the pressure-diffusion tensor $d_{i j}^{(p)}$, in wall units, plotted against the nondimensional distance from the wall $y^{+}$(in fully developed incompressible plane channel flow $\left.d_{x x}^{(p)}=d_{y z}^{(p)}=d_{z z}^{(p)}=d_{z x}^{(p)}=0\right)$.

tively), satisfying an approximate equality at the wall, which ( $c f \$ 2.2 .5$ implies that the energy-containing nondimensional wavenumbers of the source-terms $(2.3 a, 2.5 b, 2.6 a)$ are sufficiently high for the interaction between upper and lower wall to be negligible (Fig. 6). Furthermore, for the low-Reynolds-number case studied in the present work $\left(R e_{\tau_{w}} \approx 180\right)$ the Stokes pressure term $\phi_{i j}^{(\tau)}$ is of the same order-of-magnitude, in the near-wall region $\left(y^{+} \lesssim 10\right.$; Fig. 12 , as the other terms of the $p^{\prime}$-splitting $(2.10]$, and is particularly important for the shear component $\phi_{x y}$ (Fig. 12. At the wall $\left(y^{+}=0\right.$; Fig. 12 $\left[\phi_{x y}^{(\tau)}\right]_{y^{+}=0}$ accounts for $\sim 25 \%$ of $\left[\phi_{x y}\right]_{y^{+}=0}$. For a plane wall $\perp \vec{e}_{y}$, at the wall $\left(y^{+}=0\right)$, $\left[\phi_{x y}\right]_{y^{+}=0} 3.13 .2\left[\overline{p^{\prime} \partial_{y} u^{\prime}}\right]_{y^{+}=0}$ (because $\left.v_{y^{+}=0}^{\prime}=0 \Longrightarrow\left[\partial_{x} v^{\prime}\right]_{y^{+}=0}=0\right)$. Therefore, the 

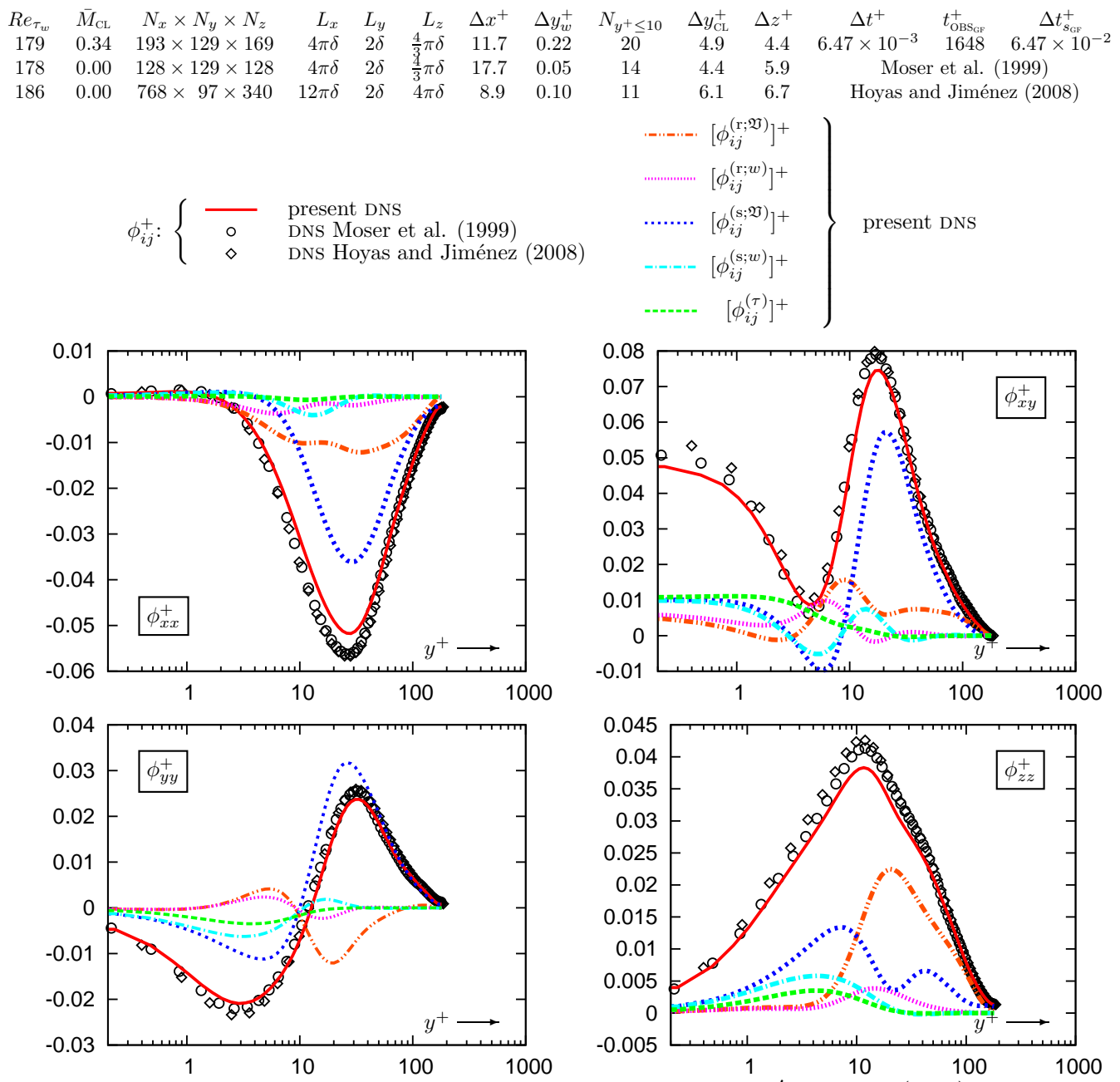

Figure 12. Distributions of the 5 terms in the incompressible $p^{\prime}$-splitting 2.10 of pressure-strain redistribution, $\phi_{i j}^{(\mathrm{r} ; \mathfrak{V})}, \phi_{i j}^{(\mathrm{r} ; w)}, \phi_{i j}^{(\mathrm{s} ; \mathfrak{V})}, \phi_{i j}^{(\mathrm{s} ; w)}$, and $\phi_{i j}^{(\tau)}$, from the present DNS computations $\left(R e_{\tau_{w}}=179 ; \bar{M}_{\mathrm{CL}}=0.34 ; 193 \times 129 \times 169\right.$ grid; Tab. 1), and distributions of pressure-strain redistribution $\phi_{i j}$ from various DNS databases (Moser et al. 1999 , Hoyas \& Jiménez 2008, Gerolymos et al. 2010), in wall units, plotted against the nondimensional distance from the wall $y^{+}$ (in statistically $2-\mathrm{D}$ plane flow $\phi_{z x}=\phi_{y z}=0$ ).

high level of $\left[\phi_{x y}^{(\tau)}\right]_{y^{+}=0}$ relative to the other terms of the $p^{\prime}$-splitting 2.10 implies that, at the wall, the fluctuating Stokes pressure $\left[p_{(\tau)}^{\prime}\right]_{y^{+}=0}$ is well correlated with the fluctuating wall-shear-stress $\left[\tau_{x y}^{\prime}\right]_{y^{+}=0}=\mu\left[\partial_{y} u^{\prime}\right]_{y^{+}=0}$. Notice that none of known models for $\phi_{i j}$ (Craft \& Launder 1996; Gerolymos \& Vallet 2001; Jakirlić \& Hanjalić 2002; Suga $2004)$ gives the correct viscous sublayer behaviour of $\phi_{x y}$ and $\phi_{y y}\left(y^{+} \lesssim 5\right.$; Fig. 12$)$. In fact, all known second-moment closures, actually model $\Pi_{i j}=\phi_{i j}+d_{i j}^{(p)}$ in the viscous sublayer (Mansour et al. 1988), where $\phi_{i j}$ (Fig. 12 and $d_{i j}^{(p)}$ (Fig. 11) cancel one another, since $\left[\Pi_{i j}\right]_{w}=0$, even when they separately model $\phi_{i j}$ and $d_{i j}^{(p)}$ (Gerolymos et al. 2012b) further away from the wall $\left(y^{+} \lesssim 5\right)$. 

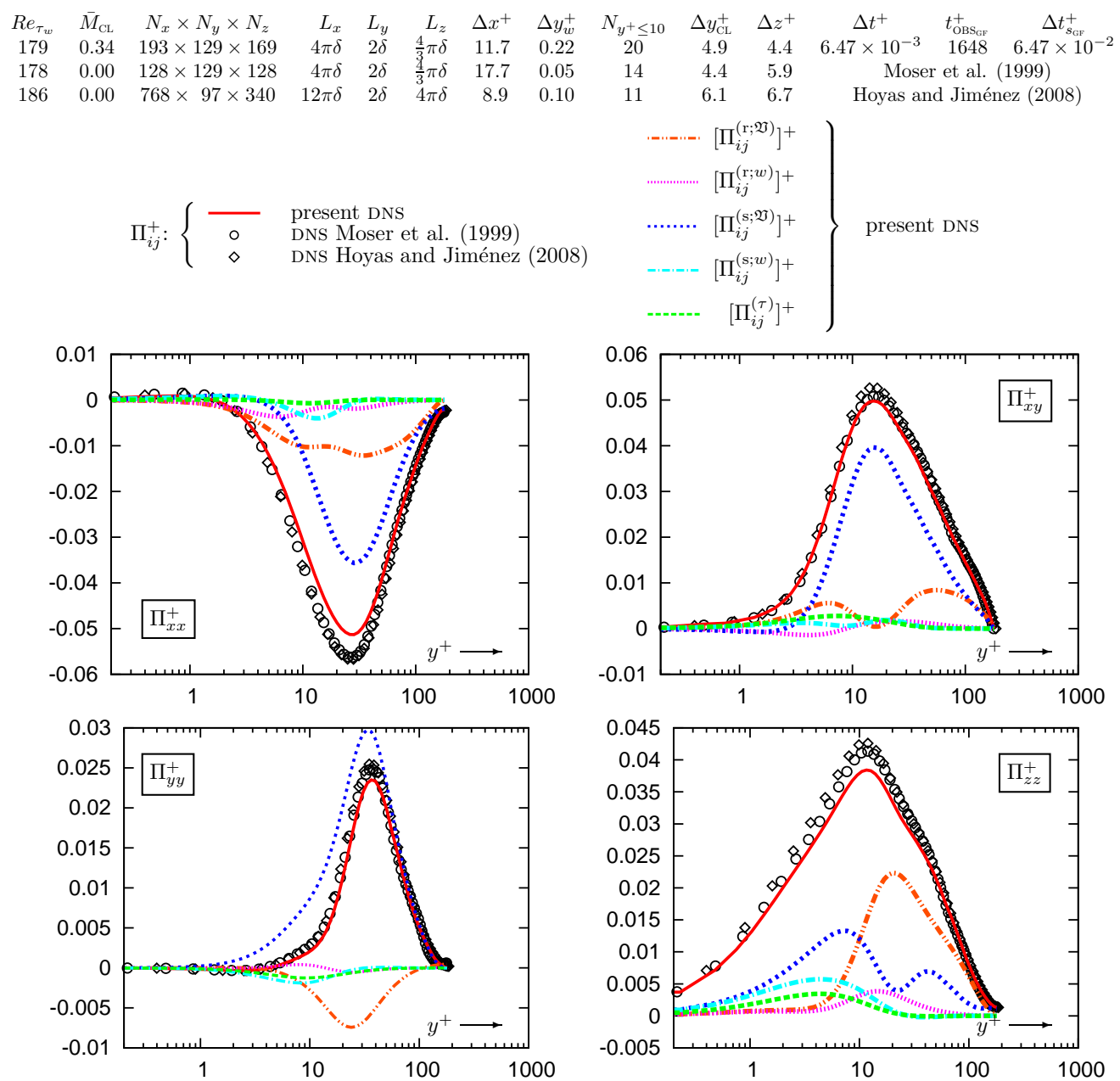

Figure 13. Distributions of the 5 terms in the incompressible $p^{\prime}$-splitting 2.10 of velocity/pressure-gradient correlation, $\Pi_{i j}^{(\mathrm{r} ; \mathfrak{Y})}, \Pi_{i j}^{(\mathrm{r} ; w)}, \Pi_{i j}^{(\mathrm{s} ; \mathfrak{Y})}, \Pi_{i j}^{(\mathrm{s} ; w)}$, and $\Pi_{i j}^{(\tau)}$, from the present DNS computations $\left(\operatorname{Re}_{\tau_{w}}=179 ; \bar{M}_{\mathrm{CL}}=0.34 ; 193 \times 129 \times 169\right.$ grid; Tab. 1), and distributions of velocity/pressure-gradient correlation $\Pi_{i j}$ from various DNs databases (Moser et al. 1999 | Hoyas \& Jiménez 2008, Gerolymos et al. 2010), in wall units, plotted against the nondimensional distance from the wall $y^{+}$(in fully developed incompressible plane channel flow $\Pi_{z x}=\Pi_{y z}=0$, $\Pi_{x x}=\phi_{x x}$, and $\left.\Pi_{z z}=\phi_{z z}\right)$.

\subsection{Velocity/pressure-gradient $\Pi_{i j}$}

The velocity/pressure-gradient correlation $\Pi_{i j}(3.2)$ is exactly the sum of pressure diffusion $d_{i j}^{(p)}\left(\S 3.3\right.$ and pressure-strain redistribution $\phi_{i j}(\$ 3.4$. By definition 3.2$)\left[\Pi_{i j}\right]_{y^{+}=0}$ because of the no-slip wall boundary-condition for the fluctuating velocity $\left[u_{i}^{\prime}\right]_{y^{+}=0}=0$, implying $\left[\phi_{i j}\right]_{y^{+}=0}=-\left[d_{i j}^{(p)}\right]_{y^{+}=0}$, so that very near the wall we expect that $\Pi_{i j}:=$ $\phi_{i j}+d_{i j}^{(p)}$ will be asymptotically approaching 0 . Observation of DNS data $\left(y^{+} \lesssim 5\right.$; Fig. 13 ) indicates that the rate at which $\Pi_{z z}$ approaches 0 as $y^{+} \rightarrow 0$ is much slower compared to the other components $\left(\Pi_{x x}, \Pi_{x y}\right.$, and $\left.\Pi_{y y}\right)$.

In fully developed incompressible plane channel flow $d_{x x}^{(p)}=d_{z z}^{(p)}=0$ by $(3.2)$, implying that $\Pi_{x x}=\phi_{x x}$ and $\Pi_{z z}=\phi_{z z}$, but also that $\Pi_{\ell \ell}=d_{y y}^{(p)}$ (since $\phi_{\ell \ell} \stackrel{3.2}{=} 0$ by the 
incompressible fluctuating continuity equation $\partial_{x_{\ell}} u_{\ell}^{\prime}=0$ ). For the three components, $\Pi_{x x}, \Pi_{x y}$ and $\Pi_{y y}$, the dominant contribution from the mechanisms of the $p^{\prime}$-splitting 2.10 comes from the slow volume terms $\left(\Pi_{x x}^{(\mathrm{s} ; \mathfrak{V})}, \Pi_{x y}^{(\mathrm{s} ; \mathfrak{V})}\right.$, and $\left.\Pi_{y y}^{(\mathrm{s} ; \mathfrak{V})}\right)$, the rapid volume terms $\left(\Pi_{x x}^{(\mathrm{r} ; \mathfrak{V})}, \Pi_{x y}^{(\mathrm{r} ; \mathfrak{V})}\right.$, and $\Pi_{y y}^{(\mathrm{r} ; \mathfrak{V})}$ ) being the main remaining contribution (Fig. 13). On the contrary, all of the 5 mechanisms of $p^{\prime}$-generation in 2.10 contribute, with the same order-of-magnitude in the near-wall region $\left(y^{+} \lesssim 10\right.$; Fig. 13$)$ to $\Pi_{z z}\left(=\phi_{z z}\right.$ in fully developed incompressible plane channel flow), this difference between $\Pi_{z z}$ and the other components being related to the slower rate at which $\Pi_{z z}$ goes to 0 as $y^{+} \rightarrow 0\left(y^{+} \lesssim 5\right.$; Fig. 13. Furthermore, contrary to $\phi_{i j}$ (Fig. 12 and $d_{i j}^{(p)}$ (Fig. 11), for which the volume and wall-echo terms of each field (rapid or slow) are of the same sign, almost everywhere, and exhibit the same variation with $y^{+}$, this behaviour applies only to $\Pi_{x x}$ and $\Pi_{z z}$. For the normal-to-the-wall $\Pi_{y y}$ and the shear $\Pi_{x y}$ components, the wall-echo and volume terms of each field (rapid or slow) are of opposite sign $\dagger$ This is not incompatible with their near-equality at the wall, where all components of $\Pi_{i j}$ vanish, because their $y$ gradients can be different. This observation is significant for near-wall modelling, because the term which appears in the Reynolds-stress transport equation is $\Pi_{i j}$, and whether $\phi_{i j}$ or $d_{i j}^{(p)}$ are modelled separately (Gerolymos et al. 2012b) or together (Mansour et al. 1988), it is the modelled sum which defines the quality of the model.

In order to explain the different behaviour of the various parts of the $p^{\prime}$-splitting $\left(p_{(\mathrm{r} ; \mathfrak{V})}^{\prime}\right)$ $\left.p_{(\mathrm{r} ; w)}^{\prime}, p_{(\mathrm{s} ; \mathfrak{V})}^{\prime}, p_{(\mathrm{s} ; w)}^{\prime}\right)$ between $\Pi_{x x}$ and $\Pi_{z z}$ on one hand, and $\Pi_{y y}$ and $\Pi_{x y}$ on the other (\$3.5), consider the analytical expression (hence transfer function) relating different parts of the $p^{\prime}$-splitting 2.10 to the rapid and slow sources $2.3 a$. Let $m \in\{\mathrm{s}, \mathrm{r}\}$ denote the rapid or slow fields, and $n \in\{\mathfrak{V}, w\}$ denote the corresponding volume or wall parts. Then, combining the $x z$-Fourier-transform representation of $p_{(m ; n)}^{\prime} 2.5 a$ and $Q_{(m)}^{\prime} 2.5 b$ with the Green's function solution of the corresponding field 2.12, 2.15),

$$
\begin{aligned}
& p_{(m ; n)}^{\prime}(x, y, z, t) \\
& \int_{-\infty}^{+\infty} \int_{-\infty}^{+\infty}\left(\int_{-\frac{1}{2} L_{y}}^{+\frac{1}{2} L_{y}} G_{n}(y, Y ; \kappa) \hat{Q}_{(m)}^{\prime}\left(\kappa_{x}, Y, \kappa_{z}, t\right) d Y\right) e^{i \kappa_{x} x+i \kappa_{z} z} d \kappa_{x} d \kappa_{z}
\end{aligned}
$$

and, by direct differentiation and averaging of $(3.3 a)$, we have

$$
\begin{aligned}
& {\left[\Pi_{x x}\right]_{(m ; n)} \stackrel{3.2}{=}-2 \overline{u^{\prime} \frac{\partial p^{\prime}}{\partial x}} \stackrel{3.3 a}{-}} \\
& -2 u^{\prime} \int_{-\infty}^{+\infty} \int_{-\infty}^{+\infty} \int_{-\frac{1}{2} L_{y}}^{+\frac{1}{2} L_{y}} G_{n}(y, Y ; \kappa) \hat{Q}_{(m)}^{\prime}\left(\kappa_{x}, Y, \kappa_{z}, t\right) i \kappa_{x} e^{i \kappa_{x} x+i \kappa_{z} z} d \kappa_{x} d \kappa_{z} d Y \\
& {\left[\Pi_{y y}\right]_{(m ; n)} \stackrel{[3.2}{=}-2 v^{\prime} \frac{\partial p^{\prime}}{\partial y} \stackrel{3.3 a}{-}} \\
& -2 v^{\prime} \int_{-\infty}^{+\infty} \int_{-\infty}^{+\infty} \int_{-\frac{1}{2} L_{y}}^{+\frac{1}{2} L_{y}} \frac{\partial G_{n}}{\partial y}(y, Y ; \kappa) \hat{Q}_{(m)}^{\prime}\left(\kappa_{x}, Y, \kappa_{z}, t\right) e^{i \kappa_{x} x+i \kappa_{z} z} d \kappa_{x} d \kappa_{z} d Y
\end{aligned}
$$

$\dagger$ Actually, in agreement with Manceau et al. (2001), all components of $\phi_{i j}$ (Fig. 12) are increased in absolute value by wall-echo, and so are the components of $d_{i j}^{(p)}$ (Fig. 11). This observation is true both for the rapid and slow fields (Figs. 11, 12, but with different factors depending on the particular component and $y^{+}$. Therefore, when these $\phi_{i j}$ and $d_{i j}^{(p)}$ are combined together, their sum $\Pi_{i j} \stackrel{[3.2]}{=} \phi_{i j}+d_{i j}^{(p)}$ behaves differently. 


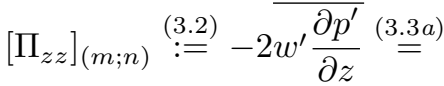

$$
\begin{aligned}
& -2 w^{\prime} \int_{-\infty}^{+\infty} \int_{-\infty}^{+\infty} \int_{-\frac{1}{2} L_{y}}^{+\frac{1}{2} L_{y}} G_{n}(y, Y ; \kappa) \quad \hat{Q}_{(m)}^{\prime}\left(\kappa_{x}, Y, \kappa_{z}, t\right) i \kappa_{z} e^{i \kappa_{x} x+i \kappa_{z} z} d \kappa_{x} d \kappa_{z} d Y \\
& {\left[\Pi_{x y}\right]_{(m ; n)} \stackrel{3.2}{=}-\overline{u^{\prime} \frac{\partial p^{\prime}}{\partial y}}-\overline{v^{\prime} \frac{\partial p^{\prime}}{\partial x}} \stackrel{3.3 a a}{=}} \\
& -\overline{v^{\prime} \int_{-\infty}^{+\infty} \int_{-\infty}^{+\infty} \int_{-\frac{1}{2} L_{y}}^{+\frac{1}{2} L_{y}} G_{n}(y, Y ; \kappa) \quad \hat{Q}_{(m)}^{\prime}\left(\kappa_{x}, Y, \kappa_{z}, t\right) i \kappa_{x} e^{i \kappa_{x} x+i \kappa_{z} z} d \kappa_{x} d \kappa_{z} d Y} \\
& -\overline{u^{\prime} \int_{-\infty}^{+\infty} \int_{-\infty}^{+\infty} \int_{-\frac{1}{2} L_{y}}^{+\frac{1}{2} L_{y}} \frac{\partial G_{n}}{\partial y}(y, Y ; \kappa) \hat{Q}_{(m)}^{\prime}\left(\kappa_{x}, Y, \kappa_{z}, t\right) \quad e^{i \kappa_{x} x+i \kappa_{z} z} d \kappa_{x} d \kappa_{z} d Y}
\end{aligned}
$$

It is obvious from (3.3) that there is a fundamental difference in the way the $x z$-Fouriercomponents of the sources interact with $u_{i}^{\prime}$ to built the components of $\Pi_{i j}$. For $\left[\Pi_{x x}\right]_{(m ; n)}$ (3.3b) and $\left[\Pi_{z z}\right]_{(m ; n)}(3.3 d)$, the contribution of each wavenumber is weighted by $i \kappa_{x}$ or $i \kappa_{z}$ (this includes not only modulus weighting but also phase-shift) and by the appropriate Green's function. This is no longer the case for $\left[\Pi_{y y}\right]_{(m ; n)}(3.3 c)$, where there is no wavenumber-weighting and the $y$-gradient of the Green's function appears. Therefore, contributions of the sources from different $Y$-locations and different wavenumbers are weighted differently for $\left[\Pi_{y y}\right]_{(m ; n)}$ compared to the other normal components, $\left[\Pi_{x x}\right]_{(m ; n)}$ and $\left[\Pi_{z z}\right]_{(m ; n)}$. This explains why the behaviour of the different fields in $\Pi_{y y}$ (Fig. 13 ) is so profoundly different compared to $\Pi_{x x}$ or $\Pi_{z z}$. Finally, $\Pi_{x y}$ (3.3e contains both types of integrals, and is arguably more complex to analyze. Similar analysis, based on $2.5 \mathrm{c}$ 2.8), applies to the Stokes field $p_{(\tau)}^{\prime}$.

The above analysis hints at the reasons of the different relative behaviour of the contribution of different pressure-fluctuation fields $\left(p_{(\mathrm{r} ; \mathfrak{V})}^{\prime}, p_{(\mathrm{r} ; w)}^{\prime}, p_{(\mathrm{s} ; \mathfrak{V})}^{\prime}, p_{(\mathrm{s} ; w)}^{\prime}\right)$ in the various components of $\Pi_{i j}$. On the other hand, (3.3) suggests that further research is required, studying specifically the spectral behaviour of $\hat{Q}_{(m)}^{\prime}$ and its convolution with $u_{i}^{\prime} x z$ Fourier-components, continuing the analysis of Chang et al. (1999), which focused on $p^{\prime}$ alone.

\section{Conclusions}

In the present paper, we consider fully developed ( $x$-wise invariant in the mean) turbulent plane channel flow. Examination of the DNS data of Hoyas \& Jiménez (2008), in the range $R e_{\tau_{w}} \in[180,2000]$, indicate that, despite the pronounced dependence of $\left[p_{w}^{\prime}\right]_{\mathrm{rms}}^{+}$on $R e_{\tau_{w}}$, the $y$-distribution of the ratio of slow-to-rapid pressure fluctuations, $\left[p_{(r)}^{\prime}\right]_{\mathrm{rms}}^{-1}\left[p_{(s)}^{\prime}\right]_{\mathrm{rms}}$, is reasonably independent of $R e_{\tau_{w}}$, particularly in the near-wall region $\left(y^{+} \lesssim 50\right)$, but also in the outer region $\left(\frac{2}{10} \delta \leq y-y_{w} \leq \frac{7}{10} \delta\right)$ where it takes a nearly constant value $(\sim 1.6)$.

The Green's function $\left(G_{\text {Kim }}\right)$ approach of $\operatorname{Kim}(1989)$, for the solution of the 1-D modified Helmholtz equation for each parallel-to-the-wall wavenumber, used in DNS computations to separate instantaneous pressure fluctuations $p^{\prime}$ into rapid, slow and Stokes terms $\left.\left(p^{\prime}=p_{(r)}^{\prime}+p_{(}^{\prime} s\right)+p_{(\tau)}^{\prime}\right)$, was extended so as to separate volume $\left(G_{\mathfrak{V}}\right)$ and wall-echo $\left(G_{w}\right)$

terms $\left(p^{\prime}=p_{(\mathrm{r} ; \mathfrak{V})}^{\prime}+p_{(\mathrm{r} ; w)}^{\prime}+p_{(\mathrm{s} ; \mathfrak{V})}^{\prime}+p_{(\mathrm{s} ; w)}^{\prime}+p_{(\tau)}^{\prime}\right)$, corresponding to volume and surface integrals in the formal solution of the the Poisson equation for $p^{\prime}$ (Chou 1945). The algorithm is based on appropriate Green's functions for the volume and wall-echo terms 
$\left(G_{\text {Kim }}=G_{\mathfrak{V}}+G_{w}\right.$; Appendix B), and is directly applicable to existing DNs databases containing flowfields sampled at different instants.

The method of images can be represented by an equivalent Green's function $\left(G_{\mathrm{MWL}}\right)$ whose expression proves that this method is the superposition (the modified Helmholtz equation for each wavenumber is linear) of the volume terms $\left(G_{\mathfrak{V}}\right)$ and of the wallecho effect $\left(G_{w_{ \pm}}\right)$that each wall would induce in the absence of the other $\left(G_{\mathrm{MWL}}=\right.$ $\left.G_{\mathfrak{V}}+G_{w_{+}}+G_{w_{-}}\right)$. The approximation of the method of images consists in neglecting the interaction between upper and lower walls, whose effect is shown by the present exact theory to amplify wall-echo, increasingly so as the nondimensional wavenumber $\kappa \delta$ increases. Theoretical analysis indicates that the method of images is a high-wavenumber approximation, the approximation error growing exponentially with $(\kappa \delta)^{-1}$, but remaining reasonable for the usual wavenumbers of energy-containing structures (error $\lesssim 1 \%$ for $\ell_{\kappa}:=2 \pi \kappa^{-1} \leq 4 \delta$ ), consistently with the generally accepted similarity between the near-wall structure of boundary-layer and plane channel flows. These results are obtained by studying the Green's functions, and as such are independent of the particular distribution of the source-terms (hence independent of $R e_{\tau_{w}}$ or $\bar{M}_{\mathrm{CL}}$ ), depending only on the wavenumber. Nonetheless, although the error estimate qualifies the method of images as a good engineering approximation, the present exact theory is preferable for the fine analysis of wall-echo effects, to avoid distorsion of the large-structures part of the spectrum, especially as it introduces no computational cost overhead.

Analysis of the $p^{\prime}$-splitting for low $R e_{\tau_{w}}=180$ flow indicates that:

(a) At the wall, the instantaneous volume $\left(p_{(\mathrm{r} ; \mathfrak{V})}^{\prime}\right.$ and $\left.p_{(\mathrm{s} ; \mathfrak{V})}^{\prime}\right)$ and wall-echo $\left(p_{(\mathrm{r} ; w)}^{\prime}\right.$ and $\left.p_{(\mathrm{s} ; w)}^{\prime}\right)$ terms of the same field (rapid or slow) are approximately equal $\left(\left[p_{(\mathrm{r} ; \mathfrak{V})}^{\prime}\right]_{w} \approx\right.$ $\left[p_{(\mathrm{r} ; w)}^{\prime}\right]_{w}$ and $\left.\left[p_{(\mathrm{s} ; \mathfrak{V})}^{\prime}\right]_{w} \approx\left[p_{(\mathbf{s} ; w)}^{\prime}\right]_{w}\right)$ but this approximate equality only holds very near the wall $\left(y^{+} \lesssim 3\right)$.

(b) Although this approximate equality between corresponding volume and wall-echo terms and similar near-wall behaviour $\left(y^{+} \lesssim 20\right)$ holds for $\phi_{i j}, \overline{p^{\prime} u_{i}^{\prime}}$ and $d_{i j}^{(p)}$, this does not apply to the velocity/pressure-gradient correlation $\Pi_{i j}$. For the $\Pi_{y y}$ and $\Pi_{x y}$ components, wall-echo opposes (opposite sign) corresponding volume terms in the range $y^{+} \lesssim 20$, contrary to $\Pi_{x x}$ and $\Pi_{z z}$. An explanation of this new finding can be sought in the way the $x z$-Fourier components of the sources are weighted by wavenumber and Green's function or its $y$-gradient in the integrals representing the gradients $\partial_{x} p^{\prime}, \partial_{y} p^{\prime}$ and $\partial_{z} p^{\prime}$.

Theoretical analysis (Appendix A) of compressibility effects in low-Mach-number flow of air shows that the additional compressible terms in the Poisson equation for $p^{\prime}$ scale with density fluctuations and spatial variations. DNs-based assessment of the order-ofmagnitude of the extra compressible terms indicates that they can be reasonably neglected for centerline Mach-number $\bar{M}_{\mathrm{CL}} \lesssim 0.35$, and that Morkovin's hypothesis stating that the leading-order effect of compressibility on turbulence is related to mean-density variation ( $\lesssim 1.5 \%$ for $\left.\bar{M}_{\mathrm{CL}} \cong 0.35\right)$ applies to the fluctuating-pressure field structure.

The main perspectives of this work are (a) The application of the algorithm to higher $R e_{\tau_{w}}$ flows, (b) The detailed spectral analysis of $\Pi_{i j}$ and the use of the data in improving near-wall modelling of pressure correlations, and (c) The application of the algorithm to the compressible Poisson equation for $p^{\prime}$ at higher (supersonic) $\bar{M}_{\mathrm{CL}}$ flows.

The authors are listed alphabetically. We are particularly grateful to Prof. B.A. Younis for many enlightening discussions. Computations were performed using HPC resources from GENCI-IDRIS (Grant 2010-022139). Sourcefiles of the code used and computer routines for the splitting are available as part of the freeware project aerodynamics (Gerolymos \& Vallet|2009, http://sourceforge.net/projects/aerodynamics). Tabulated data 
are available at http://www.aerodynamics.fr/DNS_database/CT_chnnl. The present work was partly supported by the EU-funded research project ProBand, (STREP-FP6 AST4-CT-2005-012222).

\section{Appendix A. Compressibility effects at the low-Mach-number limit}

Since the present DNS database was obtained using a compressible solver Gerolymos et al. 2010 ) at $\bar{M}_{\mathrm{CL}} \approx 0.34$, we examine here in more detail the effects of compressibility on fluctuating pressure $p^{\prime}$, and discuss in particular the parameter that should be used in the scaling of the compressibility effects. This is also important in assessing how the incompressible flow limit is approached for low-Mach-number aerodynamic flows (Durran 1989), as far as correlations containing the fluctuating pressure are concerned.

\section{A.1. Compressible flow Poisson equation for $p^{\prime}$}

The flow is modelled by the compressible Navier-Stokes equations (Gerolymos et al.|2010, (34-37), pp. 785-786). Taking the divergence of the momentum equation (Gerolymos et al. 2010, (34b), p. 785) readily yields

$$
\frac{\partial^{2}}{\partial x_{i} \partial t}\left(\rho u_{i}\right)+\frac{\partial^{2}}{\partial x_{i} \partial x_{j}}\left(\rho u_{i} u_{j}\right)=-\nabla^{2} p+\frac{\partial^{2} \tau_{i j}}{\partial x_{i} \partial x_{j}}+\frac{\partial}{\partial x_{i}}\left(\rho f_{\mathrm{V}_{i}}\right)
$$

where $f_{\mathrm{V}_{i}}$ is the body-acceleration.

Pantano \& Sarkar (2002) used the inviscid form of A 1a), considering only acoustic pressure $\left(d p_{\mathrm{a}}=a^{2} d \rho\right.$ where $a$ is the speed of sound), to study compressibility effects in high-speed shear-layers. Foysi et al. (2004) further developed (A 1a) using the Garrick operator (Garrick 1957) $\left[D_{c_{t}}\right]^{2}$ of theoretical compressible unsteady aerodynamics and aeroacoustics (Miles 1959, Bisplinghoff \& Ashley 1962), which highlights a wavelike influence of the fluctuating density, and Mahle et al. (2007) applied it to high-speed compressible mixing-layers All these high-Mach-number studies used Favre decomposition $($ Favre $1965 a b)$, and the form $\partial_{x_{i} x_{j}}^{2}\left(u_{i}^{\prime \prime} u_{j}^{\prime \prime}-\overline{u_{i}^{\prime \prime} u_{j}^{\prime \prime}}\right)$ for the slow terms. Since we are interested here in establishing the order-of-magnitude of compressibility effects in comparison with the incompressible flow equation (1.1), we recast the fluctuating part of (A 1a) in a form containing (1.1) plus compressible terms. Furthermore, the point can be made that the most general scaling of compressibility effects are density-fluctuations $\rho_{\text {rms }}^{\prime}:=\sqrt{\overline{\rho^{\prime 2}}}$; therefore, all compressible terms are rewritten in terms of $\rho$ gradients and fluctuations. This is in particular the case for correlations related to the dilatation (Ristorcelli 1997), which can be expressed using the continuity equation

$$
\frac{\partial \rho}{\partial t}+\frac{\partial \rho u_{\ell}}{\partial x_{\ell}}=0 \quad \Theta:=\operatorname{div} \vec{V}=\partial_{x_{\ell}} u_{\ell} \quad \Theta=-\frac{1}{\rho} \frac{D \rho}{D t}
$$

where $D_{t}(\cdot):=\partial_{t}()+.u_{\ell} \partial_{x_{\ell}}($.$) is the substantial derivative (Pope 2000, p. 13). Then$ (A 1a) reads

$$
\begin{aligned}
& \nabla^{2} p \stackrel{\mathrm{A} 1 a] \mathrm{A} 1 b}{\frac{\partial^{2} \tau_{i j}}{\partial x_{i} \partial x_{j}}}+\frac{\partial}{\partial x_{i}}\left(\rho f_{\mathrm{V}_{i}}\right)-\rho \frac{\partial u_{i}}{\partial x_{j}} \frac{\partial u_{j}}{\partial x_{i}} \\
& +\underbrace{\rho \frac{D}{D t}\left(\frac{1}{\rho} \frac{D \rho}{D t}\right)}_{\left[\begin{array}{ll}
\mathrm{A} 1 b \\
-
\end{array}-\rho \frac{D \Theta}{D t}\right.}-\frac{D \vec{V}}{D t} \cdot \operatorname{grad} \rho
\end{aligned}
$$


by straightforward computation $\uparrow$ using the continuity equation $(\mathrm{A} 1 \mathrm{~b})$. Substracting from A 1c its Reynolds-average readily yields the working form of the compressible flow Poisson equation for the fluctuating static pressure $p^{\prime}$

$$
\begin{aligned}
& \nabla^{2} p^{\prime}=\underbrace{\left[-\bar{\rho}\left(\frac{\partial u_{i}^{\prime}}{\partial x_{j}} \frac{\partial u_{j}^{\prime}}{\partial x_{i}}-\overline{\frac{\partial u_{i}^{\prime}}{\partial x_{j}} \frac{\partial u_{j}^{\prime}}{\partial x_{i}}}\right)\right]}_{Q_{(\mathrm{s})}^{\prime}}+\underbrace{\left[-\left(\rho^{\prime} \frac{\partial u_{i}^{\prime}}{\partial x_{j}} \frac{\partial u_{j}^{\prime}}{\partial x_{i}}-\overline{\rho^{\prime} \frac{\partial u_{i}^{\prime}}{\partial x_{j}} \frac{\partial u_{j}^{\prime}}{\partial x_{i}}}\right)\right]}_{Q_{\left(\rho^{\prime} ; \mathrm{s}\right)}^{\prime}} \\
& +\underbrace{\left[-2 \bar{\rho}\left(\frac{\partial u_{i}^{\prime}}{\partial x_{j}} \frac{\partial \bar{u}_{j}}{\partial x_{i}}\right)\right]}_{Q_{(\mathrm{r})}^{\prime}}+\underbrace{\left[-2\left(\rho^{\prime} \frac{\partial u_{i}^{\prime}}{\partial x_{j}}-\overline{\rho^{\prime} \frac{\partial u_{i}^{\prime}}{\partial x_{j}}}\right) \frac{\partial \bar{u}_{j}}{\partial x_{i}}\right]}_{Q_{\left(\rho^{\prime} ; \mathrm{r}\right)}^{\prime}}+\underbrace{\left[-\rho^{\prime} \frac{\partial \bar{u}_{i}}{\partial x_{j}} \frac{\partial \bar{u}_{j}}{\partial x_{i}}\right]}_{Q_{\left(\rho^{\prime}\right)}^{\prime}} \\
& +\underbrace{\frac{\partial^{2} \tau_{i j^{\prime}}}{\partial x_{i} \partial x_{j}}}_{Q_{(\tau)}^{\prime}}+\underbrace{\frac{\partial}{\partial x_{i}}\left(\rho f_{\mathrm{V}_{i}}-\overline{\rho f_{\mathrm{V}_{i}}}\right)}_{Q_{(\mathrm{BF})}^{\prime}}+\underbrace{\left[\rho \frac{D}{D t}\left(\frac{1}{\rho} \frac{D \rho}{D t}\right)\right]^{\prime}}_{Q_{(\Theta)}^{\prime}}+\underbrace{\left[-\frac{D \vec{V}}{D t} \cdot \operatorname{grad} \rho\right]^{\prime}}_{Q_{(\dot{V} \nabla \rho)}^{\prime}}
\end{aligned}
$$

where

$$
\begin{gathered}
\overline{\overline{D(\cdot)}}:=\overline{\frac{\partial(\cdot)}{\partial t}+u_{j} \frac{\partial(\cdot)}{\partial x_{j}}}=\frac{\partial \overline{(\cdot)}}{\partial t}+\bar{u}_{j} \frac{\partial \overline{(\cdot)}}{\partial x_{j}}+\overline{u_{j}^{\prime} \frac{\partial(\cdot)^{\prime}}{\partial x_{j}}} \\
{\left[\frac{D(\cdot)}{D t}\right]^{\prime}:=\frac{D(\cdot)}{D t}-\frac{\overline{D(\cdot)}}{D t}=\frac{\partial(\cdot)^{\prime}}{\partial t}+\bar{u}_{j} \frac{\partial(\cdot)^{\prime}}{\partial x_{j}}+u_{j}^{\prime} \frac{\partial \overline{(\cdot)}}{\partial x_{j}}+u_{j}^{\prime} \frac{\partial(\cdot)^{\prime}}{\partial x_{j}}-\overline{u_{j}^{\prime} \frac{\partial(\cdot)^{\prime}}{\partial x_{j}}}}
\end{gathered}
$$

Obviously,

$$
\begin{aligned}
& Q_{\left(\rho^{\prime} ; \mathrm{s}\right)}^{\prime} \stackrel{\mathrm{A} 1 d}{-} \frac{\rho^{\prime}}{\bar{\rho}} Q_{(\mathrm{s})}^{\prime} \\
& Q_{\left(\rho^{\prime} ; \mathrm{r}\right)}^{\prime} \stackrel{\mathrm{A} 1 d]}{-} \frac{\rho^{\prime}}{\bar{\rho}} Q_{(\mathrm{r})}^{\prime}+2 \rho^{\prime} \frac{\partial u_{i}^{\prime}}{\partial x_{j}} \frac{\partial \bar{u}_{j}}{\partial x_{i}}
\end{aligned}
$$

\section{A.2. Quasi-incompressible flow approximations}

We start by summarizing some basic approximations for flows with very small meandensity gradients and density fluctuations, which can be termed quasi-incompressible, and include most low-Mach-number flows without important heat-transfer effects. We

$$
\begin{aligned}
& \dagger \frac{\partial^{2}}{\partial x_{i} \partial t}\left(\rho u_{i}\right)+\frac{\partial^{2}}{\partial x_{i} \partial x_{j}}\left(\rho u_{i} u_{j}\right)=\frac{\partial}{\partial t}\left(\rho \Theta+u_{i} \frac{\partial \rho}{\partial x_{i}}\right)+\frac{\partial}{\partial x_{i}}\left(u_{i} \frac{\partial \rho u_{j}}{\partial x_{j}}+\rho u_{j} \frac{\partial u_{i}}{\partial x_{j}}\right) \\
& \stackrel{\mathrm{A} 1 b}{=} \frac{\partial}{\partial t}\left(\rho \Theta+u_{i} \frac{\partial \rho}{\partial x_{i}}\right)+\left(\Theta \frac{\partial \rho u_{j}}{\partial x_{j}}+u_{i} \frac{\partial}{\partial x_{i}}\left(\frac{\partial \rho u_{j}}{\partial x_{j}}\right)+\frac{\partial \rho u_{j}}{\partial x_{i}} \frac{\partial u_{i}}{\partial x_{j}}+\rho u_{j} \frac{\partial \Theta}{\partial x_{j}}\right) \\
& \text { I II III V V VI VII } \\
& =\left(\rho \frac{\partial \Theta}{\partial t}+\Theta \frac{\partial \rho}{\partial t}+\frac{\partial u_{i}}{\partial t} \frac{\partial \rho}{\partial x_{i}}+u_{i} \frac{\partial}{\partial x_{i}}\left(\frac{\partial \rho}{\partial t}\right)\right)+\left(\Theta \frac{\partial \rho u_{j}}{\partial x_{j}}+u_{i} \frac{\partial}{\partial x_{i}}\left(\frac{\partial \rho u_{j}}{\partial x_{j}}\right)+\rho \frac{\partial u_{i}}{\partial x_{j}} \frac{\partial u_{j}}{\partial x_{i}}+u_{j} \frac{\partial u_{i}}{\partial x_{j}} \frac{\partial \rho}{\partial x_{i}}+\rho u_{j} \frac{\partial \Theta}{\partial x_{j}}\right)
\end{aligned}
$$

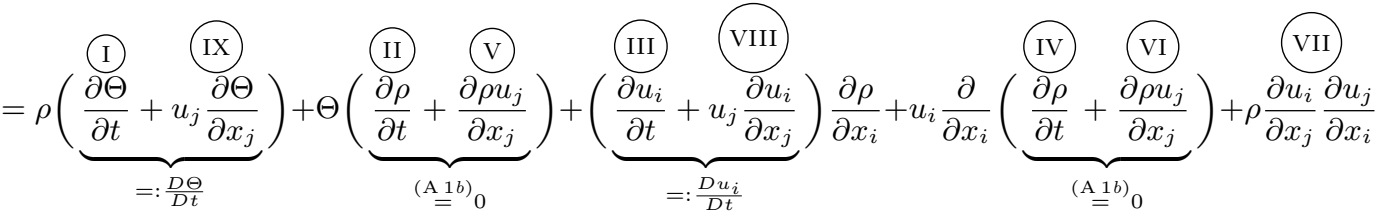


assume that for the class of flows under consideration

$$
\begin{aligned}
\frac{\rho^{\prime}}{\bar{\rho}} & \ll 1 \\
\left|\frac{\bar{D} \rho}{D t}\right| & \ll\left(\frac{D \rho}{D t}\right)_{\mathrm{rms}}^{\prime}=: \frac{\rho_{\mathrm{rms}}^{\prime}}{\mathscr{T}_{\left(D_{t} \rho\right)^{\prime}}} \stackrel{\frac{\mathrm{A}_{2} a}{\gtrless}}{\ll} \frac{\bar{\rho}}{\mathscr{T}_{\left(D_{t} \rho\right)^{\prime}}}
\end{aligned}
$$

where $\mathscr{T}_{\left(D_{t} \rho\right)^{\prime}}:=\rho_{\text {rms }}^{\prime}\left[\left(D_{t} \rho\right)_{\text {rms }}^{\prime}\right]^{-1}$ is an appropriate timescale $f i e$ density fluctuations are very small compared to mean density (A 2a), and furthermore the Reynolds-averaged substantial derivative A $1 e$ of $\rho$ is negligible compared to its fluctuating part A 1f], which is itself very small (A 2b). From (A 2a) we readily have the usual weakly compressible (Taulbee \& VanOsdol 1991) approximation

$$
\frac{1}{\rho} \stackrel{\mathrm{A} 2 a}{-} \frac{1}{\bar{\rho}}-\frac{\rho^{\prime}}{\bar{\rho}^{2}}+O\left(\frac{\rho^{2}}{\bar{\rho}^{2}}\right) \stackrel{\text { A2 } 2 a}{\simeq} \frac{1}{\bar{\rho}}-\frac{\rho^{\prime}}{\bar{\rho}^{2}}
$$

Furthermore, A 2a, A 2b imply

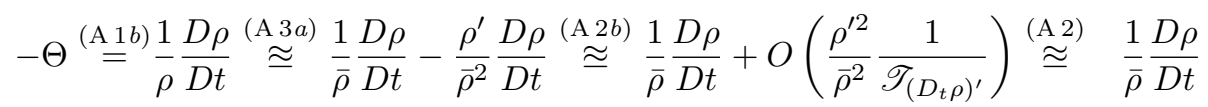

and

$$
\begin{aligned}
& -\left(\rho \frac{D \Theta}{D t}\right)^{\prime} \stackrel{\mathrm{A} 1 b}{-}\left(\rho \frac{D}{D t}\left(\frac{1}{\rho} \frac{D \rho}{D t}\right)\right)^{\prime}=\left(\frac{D^{2} \rho}{D t^{2}}-\frac{1}{\rho}\left(\frac{D \rho}{D t}\right)^{2}\right)^{\prime}
\end{aligned}
$$

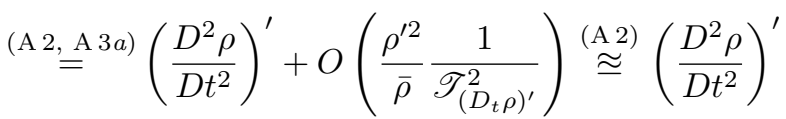

where $\mathrm{A} 2 \mathrm{~b}$ was used to establish the leading term of the error.

It was preferred to use density fluctuations and variations to establish order-of-magnitude relations, and to calculate correlations and orders-of-magnitude related to dilatation $\Theta$ via $(\mathrm{A} 1 \mathrm{~b}$, both because they are easier to understand physically than dilation , but also because they are more reliable numerically (in terms of required grid resolution and associated numerical error), the more so as $\bar{M}_{\mathrm{CL}}$ decreases.

\section{A.3. Low-Mach-number fully developed plane channel flow}

For the flow studied here (plane channel flow; $R e_{\tau_{w}}=180 ; \bar{M}_{\mathrm{CL}}=0.34$ ), conditions (A 2) leading to the approximations $(\mathrm{A} 3 \mathrm{~B}$ are satisfied. Making all quantities nondimensional in wall units $\left(\bar{\rho}_{w}, \bar{\nu}_{w}, \bar{u}_{\tau}\right)$, density variance $\left[\rho^{\prime}\right]_{\mathrm{rms}}^{+} \lesssim 2 \frac{1}{2} \times 10^{-3}$ (Fig. A1 $\mathrm{d}$ ), variance of the substantial derivative of density $\left[D_{t} \rho^{\prime}\right]_{\text {rms }}^{+} \lesssim 3 \times 10^{-4}$ (Fig. A1 d), with mean $\left[\left|\overline{D_{t} \rho}\right|\right]^{+} \lesssim$ $4 \times 10^{-5}$ (Fig. A1 ). Taking into account that $\bar{\rho}^{+} \approx 1$ with an accuracy of $1.5 \%$ (Fig. A1 a), condition (A2a) is verified as $\rho^{\prime} \sim \frac{3}{1000} \bar{\rho}$, and the separation in $\mathrm{A} 2 b$ is of 1 orderof-magnitude. Therefore (A 3) are expected to hold. Verification of (A 3a) comes from comparing $\left[\rho^{\prime}\right]_{\mathrm{rms}}^{+}$and $\left[\left(\rho^{-1}\right)^{\prime}\right]_{\mathrm{rms}}^{+}$, which are almost equal with an excellent accuracy

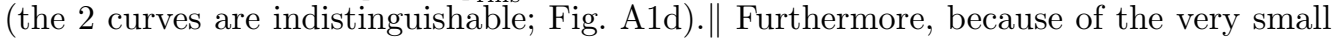

$\dagger$ It will be shown in A.3 that, for plane channel flow, this timescale made nondimensional in wall-units, satisfies $\left[\mathscr{T}_{\left(D_{t} \rho\right)^{\prime}}\right]^{+}=O(1)$

$$
\ddagger x \rightarrow 0 \Longrightarrow \frac{1}{1+x}=1-x+x^{2}-x^{3}+x^{4}+\cdots
$$

व indeed, the transport equation for the dilatation variance $D_{t} \overline{\Theta^{\prime 2}}$ is actually obtained by multiplying $\left(\mathrm{A} 1 d\right.$ by $\Theta^{\prime}$

|| although not plotted here, the same applies to skewness (opposite) and flatness (equal) of $\rho^{\prime}$ and $\left(\rho^{-1}\right)^{\prime}$ 
variation of $\bar{\rho}(y),\left[\left|d_{y} \bar{\rho}\right|\right]^{+} \lesssim 2 \times 10^{-3}=O\left(\left[\rho^{\prime}\right]_{\mathrm{rms}}^{+}\right)$(Fig. A1 $\mathrm{d}$ ) $\dagger$ the approximation $(\mathrm{A} 3 b]$ is corroborated by the excellent superposition of the DNS data for $\left[\overline{D_{t} \rho}\right]^{+}$and $\left[\overline{\rho^{-1} D_{t} \rho}\right]^{+}-\overline{\mathrm{A} 1 b}-\bar{\Theta}^{+}$(Fig. A1 . $)$.

In the case of 2-D in the mean fully developed ( $x$-wise invariant in the mean) compressible channel flow with $\vec{f}_{\mathrm{V}}=f_{\mathrm{V}_{x}}(t) \vec{e}_{x}$ (Coleman et al. 1995; Huang et al. 1995; Gerolymos et al. 2010) and using (A 1g, A 1h), A 1d made nondimensional in wall units reads

$$
\begin{gathered}
{\left[\nabla^{2} p^{\prime}\right]^{+}=\underbrace{\left(1+\frac{\left[\rho^{\prime}\right]^{+}}{\bar{\rho}^{+}}\right)\left[Q_{(\mathrm{s})}^{\prime}+Q_{(\mathrm{r})}^{\prime}\right]^{+}+2\left[\rho^{\prime} \frac{\partial v^{\prime}}{\partial x} \frac{d \bar{u}}{d y}\right]^{+}}_{\left[Q_{(\mathrm{s})}^{\prime}+Q_{\left(\rho^{\prime} ; \mathrm{s}\right)}^{\prime}+Q_{(\mathrm{r})}^{\prime}+Q_{\left(\rho^{\prime} ; \mathrm{r}\right)}^{\prime}\right]^{+}}+\underbrace{0}_{\left[Q_{\left(\rho^{\prime}\right)}^{\prime}\right]^{+}=0}} \\
+\underbrace{f_{\mathrm{V}_{x}\left[\frac{\partial \rho^{\prime}}{\partial x}\right]^{+}}+\left[Q_{(\Theta)}^{\prime}\right]^{+}+\left[Q_{(\dot{V} \nabla \rho)}^{\prime}\right]^{+}+\left[Q_{(\tau)}^{\prime}\right]^{+}}_{\left[Q_{(\mathrm{BF})}^{\prime}\right]^{+}}
\end{gathered}
$$

exactly. Statistics of the individual source-terms in A 4 were not available, but it is possible to make order-of-magnitude estimates, by relating them to available DNS data.

- $\left[Q_{(\mathrm{s})}^{\prime}+Q_{(\mathrm{r})}^{\prime}\right]^{+}$: Observation of instantaneous values indicates that $\left[Q_{(\mathrm{s})}^{\prime}+Q_{(\mathrm{r})}^{\prime}\right]^{+} \in$ $[-1,1]$, implying that the terms which were retained in the incompressible analysis 1.1 are $\left[Q_{(\mathrm{s})}^{\prime}+Q_{(\mathrm{r})}^{\prime}\right]^{+} \sim O\left(\frac{1}{10}\right)$ for the present flow conditions (plane channel flow; $R e_{\tau_{w}}=$ $\left.180 ; \bar{M}_{\mathrm{CL}}=0.34\right)$.

- $\left[Q_{\left(\rho^{\prime} ; \mathrm{s}\right)}^{\prime}\right]^{+},\left[Q_{\left(\rho^{\prime} ; \mathrm{r}\right)}^{\prime}\right]^{+},\left[Q_{\left(\rho^{\prime}\right)}^{\prime}\right]^{+}$: The term $Q_{\left(\rho^{\prime}\right)}^{\prime}$ A 1d is identically $=0$ for this flow. Obviously, the term $2 \overline{\rho^{\prime} \partial_{x} v^{\prime}} d_{y} \bar{u}=O\left(2 c_{\left(\rho^{\prime}, \partial_{x} v^{\prime}\right)} \rho_{\mathrm{rms}}^{\prime}\left(\partial_{x} v^{\prime}\right)_{\mathrm{rms}} d_{y} \bar{u}\right)$ and using the definition or $Q_{(\mathrm{r})}^{\prime} \mathrm{A} 1 d \bar{d}, 2 \overline{\rho^{\prime} \partial_{x} v^{\prime}} d_{y} \bar{u}=O\left(c_{\left(\rho^{\prime}, \partial_{x} v^{\prime}\right)} \bar{\rho}^{-1} \rho_{\mathrm{rms}}^{\prime}\left(Q_{(\mathrm{r})}^{\prime}\right)_{\mathrm{rms}}\right)$. In the present flow $\left[\left|\overline{\rho^{\prime} \partial_{x} v^{\prime}}\right| d_{y} \bar{u}\right]^{+} \lesssim 3 \times 10^{-6}$ is negligibly small (Fig. A1 $)$ )

- $\left[Q_{(\mathrm{BF})}^{\prime}\right]^{+}$: Concerning the body-force term $\left[Q_{(\mathrm{BF})}^{\prime}\right]^{+} \mathrm{A} 4$, it obviously scales with $\left[\rho^{\prime}\right]_{\text {rms }}^{+}$. It also scales with $R e_{\tau_{w}}^{-1}$ (decreases with increasing $R e_{\tau_{w}}$ ), because volumeintegration and subsequent averaging of the momentum equation (Gerolymos et al. 2010, (34b), p. 785), yields the exact relation $\overline{\rho_{\mathrm{B}} f_{\mathrm{V}_{x}}}=R e_{\tau_{w}}^{-1}$, where, because of the small variation and fluctuation of density, the bulk density (Gerolymos et al. 2010 , (46a), p. 791) $\rho_{\mathrm{B}}^{+} \approx \bar{\rho}^{+} \approx 1$. Present DNS data indicate that $\left[\bar{\rho}^{-1} \rho f_{\mathrm{V}_{x}}\left(\partial_{x} \rho\right)_{\mathrm{rms}}^{\prime}\right]^{+} \lesssim 2 \times 10^{-7}$ (Fig. A1 ).

- $\left[Q_{(\dot{V} \nabla \rho)}^{\prime}\right]^{+}:$It is easy to show that the acceleration/density-gradient source-term $\left.\left[\left(D_{t} \vec{V} \cdot \operatorname{grad} \rho\right)^{\prime}\right]^{+} \mathrm{A} 1 d\right)$ should scale with $\left[\rho^{\prime}\right]_{\mathrm{rms}}+\left(\right.$ Fig. A1 d ) and $\left[\left|d_{y} \bar{\rho}\right|\right]^{+}$(Fig. A1d $)$, weighted by acceleration statistics. The DNs data indicate $\left[\left(D_{t} \vec{V} \cdot \operatorname{grad} \rho\right)^{\prime}\right]_{\mathrm{rms}}^{+} \lesssim 5 \times 10^{-5}$ (Fig. A1p).

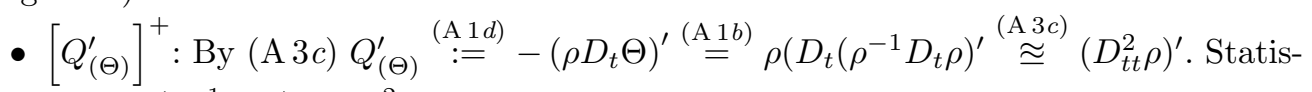
tics for $\rho D_{t}\left(\rho^{-1} D_{t} \rho\right)$ or $D_{t t}^{2} \rho$ were not available. We can make a rough estimate of its

$\dagger \dagger$ the order-of-magnitude relation between $\left[d_{y} \bar{\rho}\right]^{+}$and $\left[\rho^{\prime}\right]_{\mathrm{rms}}^{+}$can also be justified by considering the production term in the transport equation for density variance $\overline{\rho^{\prime 2}} \sqrt{\text { Taulbee \& }}$ VanOsdol 1991, (17), p. 4), which, for the flow under consideration, is $-\overline{\rho^{\prime} v^{\prime}} d_{y} \bar{\rho}$

$\left\lceil\right.$ the present DNS data indicate that the correlation coefficient $c_{\left(\rho^{\prime}, \partial_{x} v^{\prime}\right)} \approx 0.3 \pm 0.05$ in the range $y^{+} \in[1,100]$, falling to 0 at the wall and at channel centerline 


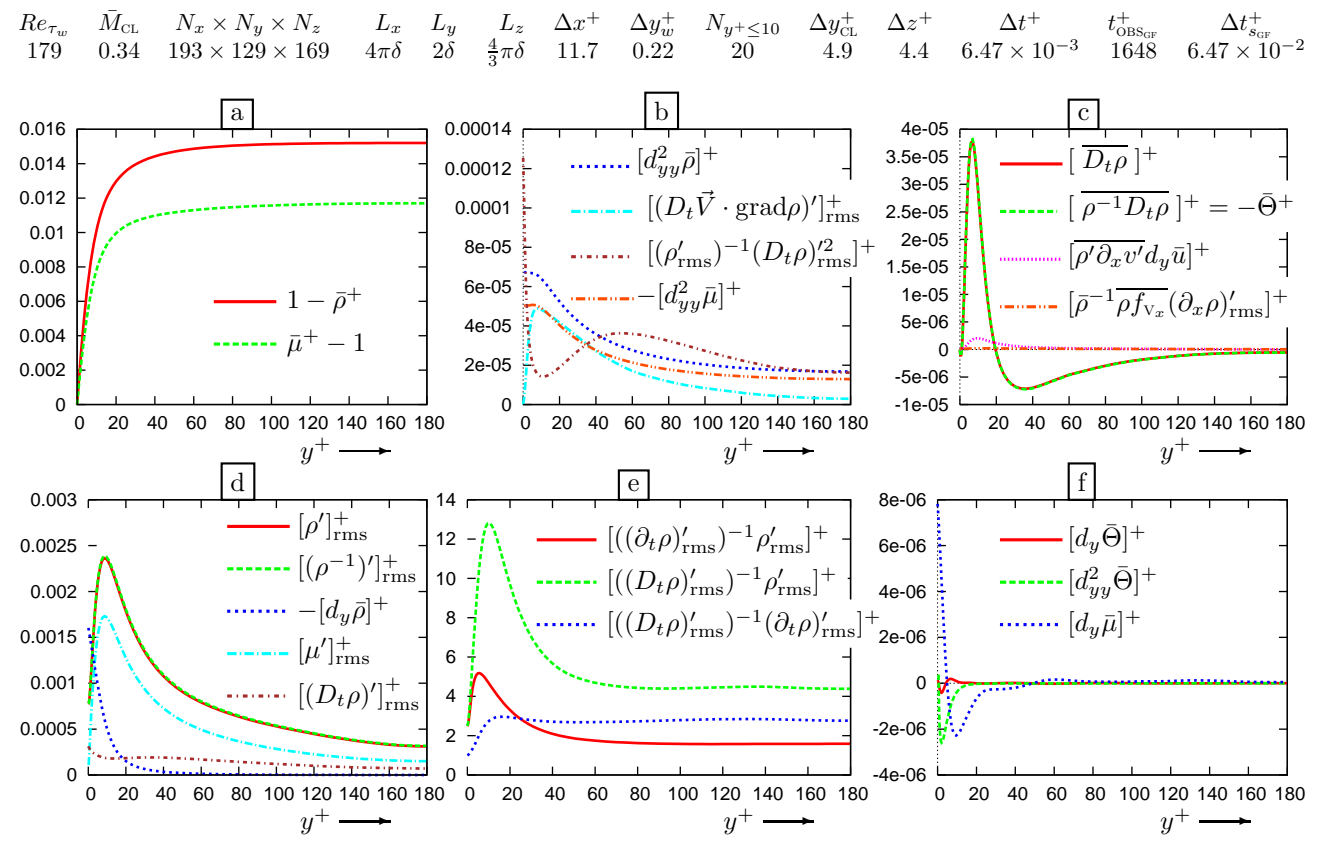

FiguRE A1. Present DNS-computed data $\left(R e_{\tau_{w}}=180 ; \bar{M}_{\mathrm{CL}}=0.34 ; 257 \times 129 \times 385\right.$ grid; Tab. 1 related to density variation and fluctuation (a) $\bar{\rho}, \bar{\mu}$, (b) $d_{y y}^{2} \bar{\rho},\left[\left(D_{t} \vec{V} \cdot \operatorname{grad} \rho\right)^{\prime}\right]_{\mathrm{rms}}$, $\left(\left(\partial_{t} \rho\right)_{\mathrm{rms}}^{\prime}\right)^{-1}\left(\left(D_{t} \rho\right)_{\mathrm{rms}}^{\prime}\right)^{2},-d_{y y}^{2} \bar{\mu}$, (c) $\overline{D_{t} \rho}, \overline{\rho^{-1} D_{t} \rho}=-\bar{\Theta}, \overline{\rho^{\prime} \partial_{x} v^{\prime}} d_{y} \bar{u}, \bar{\rho}^{-1} \overline{\rho f_{\mathrm{v} x}}\left(\partial_{x} \rho\right)_{\mathrm{rms}}^{\prime}$, (d) $\rho_{\mathrm{rms}}^{\prime}$, $\left(\rho^{-1}\right)_{\mathrm{rms}}^{\prime},-d_{y} \bar{\rho}, \mu_{\mathrm{rms}}^{\prime},\left(D_{t} \rho\right)_{\mathrm{rms}}^{\prime},(\mathrm{e})\left(\left(\partial_{t} \rho\right)_{\mathrm{rms}}^{\prime}\right)^{-1} \rho_{\mathrm{rms}}^{\prime},\left(\left(D_{t} \rho\right)_{\mathrm{rms}}^{\prime}\right)^{-1} \rho_{\mathrm{rms}}^{\prime},\left(\left(D_{t} \rho\right)_{\mathrm{rms}}^{\prime}\right)^{-1}\left(\partial_{t} \rho\right)_{\mathrm{rms}}^{\prime}$, (f) $d_{y} \bar{\Theta}, d_{y u}^{2} \Theta, d_{y} \bar{\mu}$, in wall units, relevant to the order-of-magnitude analysis of compressibility effects in $\mathrm{A} 1 \mathrm{~d})$.

order-of-magnitude. From the available statistics for $\left(D_{t} \rho\right)_{\text {rms }}^{\prime}($ Fig. A1 $\mathrm{d})$ and $\left(\partial_{t} \rho\right)_{\text {rms }}^{\prime}$ (not plotted) we can define associated timescales, $\mathscr{T}_{\left(D_{t} \rho\right)^{\prime}}:=\rho_{\text {rms }}^{\prime}\left[\left(D_{t} \rho\right)_{\text {rms }}^{\prime}\right]^{-1}$ and $\mathscr{T}_{\left(\partial_{t} \rho\right)^{\prime}}:=$ $\rho_{\text {rms }}^{\prime}\left[\left(\partial_{t} \rho\right)_{\text {rms }}^{\prime}\right]^{-1}$. These specific timescales (Fig. A1 $)$ ), associated with the fluctuation of the Eulerian and Lagrangian time-derivatives are practically constant in the major part of the channel $\left(y^{+} \gtrsim 15\right.$ ), and their ratio (Fig. A1 $)$ is $\sim 1$ near the wall where convection is small, and grows to $\sim 3$ for $y^{+} \gtrsim 15$, which is also a typical value of the ratio of Lagrangian to Eulerian timescales (Dosio et al. 2005), although these are not the timescales defined here. Then, by analogy, we assume that the same timescale $\mathscr{T}_{\left(D_{t} \rho\right)^{\prime}}$ relates the fluctuation of the time-derivative of $D_{t} \rho$ to $\left(D_{t} \rho\right)_{\mathrm{rms}}^{\prime}$, viz

$$
\left[\rho \frac{D}{D t}\left(\rho^{-1} \frac{D \rho}{D t}\right)\right]_{\mathrm{rms}}^{\prime} \stackrel{\sqrt{\mathrm{A} 3 c}}{\underset{\approx}{\simeq}}=\left(\frac{D\left(D_{t} \rho\right)}{D t}\right)_{\mathrm{rms}}^{\prime} \sim \frac{\left(D_{t} \rho\right)_{\mathrm{rms}}^{\prime}}{\mathscr{T}_{\left(D_{t} \rho\right)^{\prime}}}=O\left(\frac{\left[\left(D_{t} \rho\right)^{\prime}\right]_{\mathrm{rms}}^{2}}{\rho_{\mathrm{rms}}^{\prime}}\right)
$$

which suggests that this term is not expected to be important in the present flow, where $\left[\left(\rho_{\mathrm{rms}}^{\prime}\right)^{-1}\left[\left(D_{t} \rho\right)^{\prime}\right]_{\mathrm{rms}}^{2}\right]^{+} \lesssim 4.10^{-5}$ (Fig. A1p). Notice that the term associated with $\bar{\rho}$ in $D_{t t}^{2}$ is of the same order-of-magnitude as the estimate $\left[\mathrm{A} 5\right.$, viz $\left[\left|d_{y y}^{2} \bar{\rho}\right|\right]^{+} \lesssim 6 \times 10^{-5}$ (Fig. A1p).

- $\left[Q_{(\tau)}^{\prime}\right]^{+}$: The present calculations used a Newtonian constitutive relation (Gerolymos et al. 2010, (36a), p. 786), with $\mu_{\mathrm{b}}=0$ and $\mu=\mu(T)$ (Gerolymos et al. 2010, (37), 
p. 786). The exact expression of $Q_{(\tau)}^{\prime} \dagger$ involves terms depending on the variation and fluctuation of $\mu$ and $\Theta$. For this reason, in strictly incompressible flow, $Q_{(\tau)}^{\prime}=0$. In the present flow (plane channel flow; $R e_{\tau_{w}}=180 ; \bar{M}_{\mathrm{CL}}=0.34$ ), mean viscosity varies in a way very similar to $\rho, \bar{\mu}^{+} \approx 1$ with an accuracy of $1.2 \%$ (Fig. A1 a), and the same similarity with density applies to spatial variations, $\left[\left|d_{y} \bar{\mu}\right|\right]^{+} \lesssim 8 \times 10^{-6}$ (Fig. A1f) and $\left[\left|d_{y y}^{2} \bar{\mu}\right|\right]^{+} \lesssim 5 \times 10^{-5}$ (Fig. A1p), and fluctuation, $\left[\mu^{\prime}\right]_{\mathrm{rms}}^{+} \lesssim 2 \times 10^{-3}$ (Fig. A1 d). As already stated, mean dilatation $\left|\bar{\Theta}^{+}\right| \stackrel{\mathrm{A} 1 b \mathrm{~b}}{-}\left[\left|\overline{\rho^{-1} D_{t} \rho}\right|\right]^{+} \lesssim 4 \times 10^{-5}$, with gradients $\left[\left|d_{y} \bar{\Theta}\right|\right]^{+} \lesssim 4 \times 10^{-7}$ (Fig. A1f) and $\left[\left|d_{y y}^{2} \bar{\Theta}\right|\right]^{+} \lesssim 2 \times 10^{-6}$ (Fig. A1F), and fluctuation $\left[\Theta^{\prime}\right]_{\mathrm{rms}}^{+} \stackrel{[\mathrm{A} 3 b}{\approx}\left[D_{t} \rho^{\prime}\right]_{\mathrm{rms}}^{+} \lesssim 3 \times 10^{-4}$ (Fig. A1d). Therefore, terms containing products of the small (in wall units) quantities $d_{y} \bar{\mu}, d_{y y}^{2} \bar{\mu}, \bar{\Theta}, d_{y} \bar{\Theta}, d_{y y}^{2} \bar{\Theta}, \mu^{\prime}$ (and its space-derivatives), and $\Theta^{\prime}$ (and its space-derivatives), can be considered negligible compared to other terms, and we have $\left(\mu_{\mathrm{b}}=0\right)$

$$
Q_{(\tau)}^{\prime}=\frac{4}{3} \bar{\mu} \nabla^{2} \Theta^{\prime}+4 \frac{\partial S_{y j}^{\prime}}{\partial x_{j}} \frac{d \bar{\mu}}{d y}+2 \frac{\partial v^{\prime}}{\partial y} \frac{d^{2} \bar{\mu}}{d y^{2}}+2 \frac{\partial \mu^{\prime}}{\partial x} \frac{d^{2} \bar{u}}{d y^{2}}+2 \frac{d \bar{u}}{d y} \frac{\partial^{2} \mu^{\prime}}{\partial x \partial y}+o\left(Q_{(\tau)}^{\prime}\right)
$$

where $S_{i j}:=\frac{1}{2}\left(\partial_{x_{j}} u_{i}+\partial_{x_{i}} u_{j}\right)$ is the rate-of-strain tensor.

Using the above estimates in A1d, A 4 we may write

$$
\begin{aligned}
& \frac{\left[\rho^{\prime}\right]^{+}}{\bar{\rho}^{+}}\left[Q_{(\mathrm{s})}^{\prime}+Q_{(\mathrm{r})}^{\prime}\right]^{+}+O\left(\left(\left[\rho^{\prime}\right]_{\mathrm{rms}}^{+}\left[Q_{(\mathrm{r})}^{\prime}\right]_{\mathrm{rms}}^{+}\right)\right. \\
& {\left[\nabla^{2} p^{\prime}\right]^{+}=\left[Q_{(\mathrm{s})}^{\prime}+Q_{(\mathrm{r})}^{\prime}\right]^{+}+\overbrace{\left[Q_{\left(\rho^{\prime} ; \mathrm{s}\right)}^{\prime}+Q_{\left(\rho^{\prime} ; \mathrm{r}\right)}^{\prime}\right]^{+}}^{+0}+\overbrace{\left[Q_{\left(\rho^{\prime}\right)}^{\prime}\right]^{+}}^{=0}}
\end{aligned}
$$

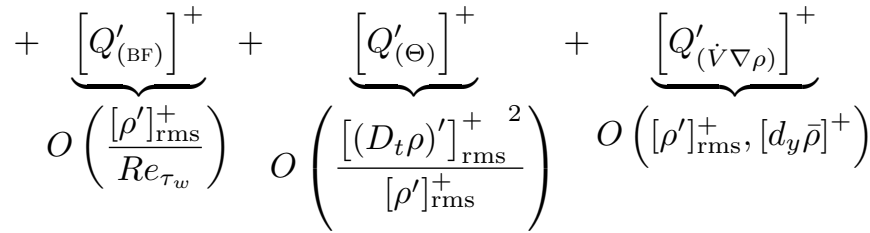

$$
\begin{aligned}
& +\underbrace{\left[Q_{(\tau)}^{\prime}\right]^{+}} \\
& O\left(\left[\left(D_{t} \rho\right)^{\prime}\right]_{\mathrm{rms}}^{+},\left[\mu^{\prime}\right]_{\mathrm{rms}}^{+},\left[d_{y} \bar{\mu}\right]^{+},\left[d_{y y}^{2} \bar{\mu}\right]^{+}\right)
\end{aligned}
$$

By (A 7) it is clear that all extra compressible terms in A 1dd), compared to (1.1), scale with $\left[\rho^{\prime}\right]_{\mathrm{rms}}^{+}$or $\left[\left(D_{t} \rho\right)^{\prime}\right]_{\mathrm{rms}}^{+}$and $\left[d_{y} \bar{\rho}\right]^{+}(\mu$ gradients and fluctuations, in wall units, being of

$$
\begin{aligned}
\dagger Q_{(\tau)}^{\prime} \stackrel{\stackrel{\mathrm{A} 1 d \mathrm{~d}}{=}}{=} \frac{\partial^{2} \tau_{i j^{\prime}}}{\partial x_{i} \partial x_{j}} \\
=\left(\left(\frac{4}{3} \mu+\mu_{\mathrm{b}}\right) \nabla^{2} \Theta+4 \frac{\partial \mu}{\partial x_{i}} \frac{\partial S_{i j}}{\partial x_{j}}+2 S_{i j} \frac{\partial^{2} \mu}{\partial x_{i} \partial x_{j}}+\Theta \nabla^{2}\left(\mu_{\mathrm{b}}-\frac{2}{3} \mu\right)+2 \frac{\partial\left(\mu_{\mathrm{b}}-\frac{2}{3} \mu\right)}{\partial x_{j}} \frac{\partial \Theta}{\partial x_{j}}\right)^{\prime} \\
=\left(\left(\frac{4}{3} \mu^{\prime}+\mu_{\mathrm{b}}^{\prime}\right) \nabla^{2} \bar{\Theta}+4 \frac{\partial \mu^{\prime}}{\partial x_{i}} \frac{\partial \bar{S}_{i j}}{\partial x_{j}}+2 S_{i j}^{\prime} \frac{\partial^{2} \bar{\mu}}{\partial x_{i} \partial x_{j}}+\Theta^{\prime} \nabla^{2}\left(\bar{\mu}_{\mathrm{b}}-\frac{2}{3} \bar{\mu}\right)+2 \frac{\partial\left(\mu_{\mathrm{b}}^{\prime}-\frac{2}{3} \mu^{\prime}\right)}{\partial x_{j}} \frac{\partial \bar{\Theta}}{\partial x_{j}}\right) \\
+\left(\left(\frac{4}{3} \bar{\mu}+\bar{\mu}_{\mathrm{b}}\right) \nabla^{2} \Theta^{\prime}+4 \frac{\partial \bar{\mu}}{\partial x_{i}} \frac{\partial S_{i j}^{\prime}}{\partial x_{j}}+2 \bar{S}_{i j} \frac{\partial^{2} \mu^{\prime}}{\partial x_{i} \partial x_{j}}+\bar{\Theta} \nabla^{2}\left(\mu_{\mathrm{b}}^{\prime}-\frac{2}{3} \mu^{\prime}\right)+2 \frac{\partial\left(\bar{\mu}_{\mathrm{b}}-\frac{2}{3} \bar{\mu}\right)}{\partial x_{j}} \frac{\partial \Theta^{\prime}}{\partial x_{j}}\right) \\
+\left(\left(\frac{4}{3} \mu^{\prime}+\mu_{\mathrm{b}}^{\prime}\right) \nabla^{2} \Theta^{\prime}+4 \frac{\partial \mu^{\prime}}{\partial x_{i}} \frac{\partial S_{i j}^{\prime}}{\partial x_{j}}+2 S_{i j}^{\prime} \frac{\partial^{2} \mu^{\prime}}{\partial x_{i} \partial x_{j}}+\Theta^{\prime} \nabla^{2}\left(\mu_{\mathrm{b}}^{\prime}-\frac{2}{3} \mu^{\prime}\right)+2 \frac{\partial\left(\mu_{\mathrm{b}}^{\prime}-\frac{2}{3} \mu^{\prime}\right)}{\partial x_{j}} \frac{\partial \Theta^{\prime}}{\partial x_{j}}\right) \\
-\left(\left(\frac{4}{3} \mu^{\prime}+\mu_{\mathrm{b}}^{\prime}\right) \nabla^{2} \Theta^{\prime}+4 \frac{\partial \mu^{\prime}}{\partial x_{i}} \frac{\partial S_{i j}^{\prime}}{\partial x_{j}}+2 S_{i j}^{\prime} \frac{\partial^{2} \mu^{\prime}}{\partial x_{i} \partial x_{j}}+\Theta^{\prime} \nabla^{2}\left(\mu_{\mathrm{b}}^{\prime}-\frac{2}{3} \mu^{\prime}\right)+2 \frac{\partial\left(\mu_{\mathrm{b}}^{\prime}-\frac{2}{3} \mu^{\prime}\right)}{\partial x_{j}} \frac{\partial \Theta^{\prime}}{\partial x_{j}}\right)
\end{aligned}
$$


the same order-of-magnitude as corresponding gradients and fluctuations of $\rho$; Fig. A1. The order-of-magnitude analysis of different terms indicates that they can be reasonably neglected for the flow studied in the present work (plane channel flow; $R e_{\tau_{w}}=180$; $\bar{M}_{\mathrm{CL}}=0.34$ ). As a consequence, the main influence of compressibility appears in the retained quasi-incompressible terms $Q_{(\mathrm{s})}^{\prime}+Q_{(\mathrm{r})}^{\prime}$ A 1d. 1.1p, through the variation of $\bar{\rho}(y)$, indicating the validity of Morkovin's hypothesis (So et al. 1998), which states that, for low-Mach-number flow, the effects of compressibility on turbulence are due to the mean-density-gradient, the influence of $\rho^{\prime}$ being a higher-order effect.

\section{Appendix B. Green's functions}

We analyze the mathematical tools for the solution of the modified Helmholtz equation (2.6) which provides the terms of the $p^{\prime}$-splitting 2.2 2.10 for each Fourier $x z$ Fourier-component of $p^{\prime}(2.5 a)$. The Green's functions approach for the solution of (2.6) is briefly summarized in $\$ \mathrm{~B} .1$. In $8 \mathrm{~B} .2$ we study the case of spatially $x z$-varying Fourier components $\left(\kappa:=\sqrt{\kappa_{x}^{2}+\kappa_{z}^{2}} \neq 0\right)$, for which the Green's function has the general form (B 9), parametrized by 2 functions, $A_{1}(Y ; \kappa)$ and $A_{2}(Y ; \kappa)$, which are determined by the boundary-conditions. Homogeneous Neumann boundary-conditions $(2.6 b)$ yield $(\$ B .2 .1)$ the well known solution of $\operatorname{Kim}(\operatorname{Kim} 1989), G_{\operatorname{Kim}}(y, Y ; \kappa \neq 0)(\mathrm{B} 10 c)$, providing the solution 2.7) for the rapid $p_{(\mathrm{r})}^{\prime}$ and slow $p_{(\mathrm{s})}^{\prime}$ parts 2.2), while the solution (Moser et al. 1999: Chang et al. 1999) for Stokes part $p_{(\tau)}^{\prime}(2.2)$, with $Q_{(\tau)}^{\prime}=0$ in strictly incompressible flow $(2.3 a, 2.6 a)$, which is driven by the inhomogeneous Neumann boundary-conditions 2.3b 2.4 2.6b), given by $q_{\mathrm{BC}}\left(y ; \kappa \neq 0, B_{ \pm}\right)(\mathrm{B} 12)$, is calculated in $\$ \mathrm{~B} .2 .2$. The free-space Green's function $G_{\mathfrak{V}}(y, Y ; \kappa \neq 0)$ (B 14), which provides the solution (2.12) for the rapid $p_{(\mathrm{r} ; \mathfrak{V})}^{\prime}$ and slow $p_{(\mathrm{s} ; \mathfrak{V})}^{\prime}$ volume-parts of the $p^{\prime}$-splitting 2.10 is calculated in $\$ \mathrm{~B} .2 .3$. Finally, for use in the analysis (2.2.4) of the method-of-images approach (Manceau et al. 2001), we calculate in $\$$ B.2.4 the Green's functions $G_{ \pm}(y, Y ; \kappa \neq 0)(\mathrm{B} 18)$ and in $\$ \mathrm{~B} .2 .5$ the boundary-conditions functions $q_{\mathrm{BC}_{ \pm}}\left(y ; \kappa \neq 0, B_{ \pm}\right)(\mathrm{B} 22)$, for the halfspace problems with only 1 (upper or lower) of the walls present. In \$B.3 we discuss the singular case $\kappa:=\sqrt{\kappa_{x}^{2}+\kappa_{z}^{2}}=0 \Longrightarrow \kappa=\kappa_{x}=\kappa_{z}=0$, for which the modified Helmholtz equation 2.6) can be solved iff the compatibility conditions (B 24) hold. We revisit ( $\mathrm{B}$.3.1 $\mathrm{B}$. 3.5 the same problems as for the $\kappa \neq 0$ case, and calculate the corresponding Green's functions $G_{\text {Kim }}(y, Y ; \kappa=0) G_{\mathfrak{V}}(y, Y ; \kappa=0)=G_{ \pm}(y, Y ; \kappa=0)$ (B 27, B 33, B 35 boundaryconditions functions $q_{\mathrm{BC}}\left(y ; \kappa=0, B_{-}, B_{+}\right)$(B32) and $q_{\mathrm{BC}_{ \pm}}\left(y ; \kappa=0, B_{ \pm}\right)$(B37), for the $\kappa=0$ case. Finally, in $\$ \mathrm{~B} .4$ we calculate the Green's function $G_{\mathrm{MwL}}(y, Y ; \kappa)$ (B 41) which corresponds to the method-of-images evaluation 2.16 of the rapid $p_{(\mathrm{r} ; \mathrm{MwL})}^{\prime}$ and

slow $p_{(\mathrm{s} ; \mathrm{MWL})}^{\prime}$ parts, and evaluate the error of the method-of-images approximation as a function of the wavenumber $(\mathrm{B} 45)$. The application of these mathematical results to the calculation of the $p^{\prime}$-splitting is described in the main paper $(22.2$.

\section{B.1. Green's function solution of the modified Helmholtz equation}

As discussed in 2.2 , we have to solve the generic modified Helmholtz (Cheng et al. 2006) equation

$$
\frac{d^{2} q(y ; \kappa)}{d y^{2}}-\kappa^{2} q(y ; \kappa)=Q(y ; \kappa) \quad y \in\left(L_{-}, L_{+}\right)
$$

with associated boundary-conditions at $y \in\left\{L_{-}, L_{+}\right\}$, for the complex-valued function $q: \mathbb{R} \longrightarrow \mathbb{C}, Q(y)$ being a given complex-valued function $Q: \mathbb{R} \longrightarrow \mathbb{C}$, and $\kappa \in \mathbb{R}_{\geq 0}$. Notice that there is no loss of generality in assuming $\kappa \in \mathbb{R}_{\geq 0}$ because (B1) depends on 
$\kappa^{2}$ and is therefore independent of the sign of $\kappa$. The general method of solution (Bender \& Orszag 1978) of the linear ODE (B 1) is based on the determination of the appropriate Green's function $G(y, Y ; \kappa)$, solution of

$$
\begin{array}{cc}
\frac{\partial^{2} G(y, Y ; \kappa)}{\partial y^{2}}-\kappa^{2} G(y, Y ; \kappa)=\delta(y-Y) & \forall y, Y \in\left[L_{-}, L_{+}\right] \\
\lim _{\epsilon \rightarrow 0^{+}}\left[\frac{\partial G}{\partial y}(y=Y+\epsilon, Y ; \kappa)-\frac{\partial G}{\partial y}(y=Y-\epsilon, Y ; \kappa)\right]=1 & \forall Y \in\left[L_{-}, L_{+}\right] \\
G(y, Y ; \kappa) \in C^{0}\left(L_{-}, L_{+}\right) & \\
G(y, Y ; \kappa) \in C^{1}\left(L_{-}, L_{+}\right) \backslash\{Y\} &
\end{array}
$$

so that

$$
q(y ; \kappa):=\int_{L_{-}}^{L_{+}} G(y, Y ; \kappa) Q(Y ; \kappa) d Y
$$

satisfies (B1) because of (B2), as can be verified by substituting (B 3) in (B1). The Green's function satisfies the symmetry (reciprocity) condition

$$
G(y, Y ; \kappa)=G(Y, y ; \kappa) \quad \forall y, Y \in\left(L_{-}, L_{+}\right) \quad \forall \kappa \in \mathbb{R}_{\geq 0}
$$

because the modified Helmholz operator $\left[d_{y y}^{2}(\cdot)-\kappa^{2}(\cdot)\right]$ is a self-adjoint linear differential operator (Ince 1926: Courant \& Hilbert 1953). As shown in Bender \& Orszag (1978), the general solution of $(\mathrm{B} 2)$ is

$$
G(y, Y ; \kappa)=\left\{\begin{array}{rr}
A_{1}(Y ; \kappa) q_{1}(y ; \kappa)+A_{2}(Y ; \kappa) q_{2}(y ; \kappa) & y \leq Y \\
\left(A_{1}(Y ; \kappa)-\frac{q_{2}(Y ; \kappa)}{\left[W\left(q_{1}, q_{2}\right)\right](Y ; \kappa)}\right) q_{1}(y ; \kappa) & \\
+\left(A_{2}(Y ; \kappa)+\frac{q_{1}(Y ; \kappa)}{\left[W\left(q_{1}, q_{2}\right)\right](Y ; \kappa)}\right) q_{2}(y ; \kappa) & y \geq Y
\end{array}\right.
$$

where $q_{1}(y ; \kappa)$ and $q_{2}(y ; \kappa)$ are 2 linearly independent solutions of the corresponding homogeneous equation $q^{\prime \prime}(y ; \kappa)-\kappa^{2} q(y ; \kappa)=0$,

$$
\left[W\left(q_{1}, q_{2}\right)\right](y ; \kappa):=\operatorname{det}\left[\begin{array}{ll}
q_{1}(y ; \kappa) & q_{2}(y ; \kappa) \\
q_{1}^{\prime}(y ; \kappa) & q_{2}^{\prime}(y ; \kappa)
\end{array}\right] \neq 0
$$

is the Wronskian $\left(.^{\prime}\right.$ in $(\mathrm{B} 6)$ denotes differentiation by $y, \kappa$ being a parameter), which is $\neq 0$ iff the 2 solutions are linearly independent. The functions $A_{1}(Y ; \kappa)$ and $A_{2}(Y ; \kappa)$ are determined by the boundary-conditions. It is straightforward to verify that $G(y, Y ; \kappa)$ (B5) is continuous at $y=Y$. Notice that by straightforward differentiation of (B 3)

$$
q^{\prime}(y ; \kappa)=\int_{L_{-}}^{L_{+}} \frac{\partial G}{\partial y}(y, Y ; \kappa) Q(Y ; \kappa) d Y
$$

The cases $\kappa \neq 0$ and $\kappa=0$ are fundamentally different, not only because the 2 linearly independent solutions of the homogeneous equation $q^{\prime \prime}(y ; \kappa)-\kappa^{2} q(y ; \kappa)=0$ differ, but principally because $\kappa=0$ is a characteristic number (eigenvalue) of $\mathrm{B} 1$, viz $q^{\prime \prime}(y ; \kappa)-$ $\kappa^{2} q(y ; \kappa) \stackrel{\kappa=0}{=} q^{\prime \prime}(y ; \kappa=0)=0$ with homogeneous Neumann boundary-conditions admits the constant function as an eigensolution (Ince 1926, Courant \& Hilbert 1953). 


\section{B.2. $\kappa \neq 0$ (xz-varying components)}

When $\kappa \neq 0$ the 2 linearly independent solutions of the homogeneous equation $q^{\prime \prime}(y ; \kappa \neq$ $0)-\kappa^{2} q(y ; \kappa \neq 0)=0$ are

$$
\begin{aligned}
& q_{1}(y ; \kappa \neq 0)=\mathrm{e}^{+\kappa y} \\
& q_{2}(y ; \kappa \neq 0)=\mathrm{e}^{-\kappa y}
\end{aligned}
$$

with Wronskian

$$
\left[W\left(q_{1}, q_{2}\right)\right](y ; \kappa \neq 0)=\operatorname{det}\left[\begin{array}{rr}
\mathrm{e}^{+\kappa y} & \mathrm{e}^{-\kappa y} \\
+\kappa \mathrm{e}^{+\kappa y} & -\kappa \mathrm{e}^{-\kappa y}
\end{array}\right]=-2 \kappa \neq 0
$$

so that solution $(\mathrm{B} 5$ reads

$$
G(y, Y ; \kappa \neq 0)=\left\{\begin{array}{rr}
A_{1}(Y ; \kappa) \mathrm{e}^{+\kappa y}+A_{2}(Y ; \kappa) \mathrm{e}^{-\kappa y} & y \leq Y \\
A_{1}(Y ; \kappa) \mathrm{e}^{+\kappa y}+A_{2}(Y ; \kappa) \mathrm{e}^{-\kappa y} & \\
+\frac{1}{\kappa} \sinh [\kappa(y-Y)] & y \geq Y
\end{array}\right.
$$

The functions $A_{1}(Y ; \kappa)$ and $A_{2}(Y ; \kappa)$ are determined so as to satisfy the boundaryconditions.

B.2.1. $G_{\operatorname{Kim}}(y, Y ; \kappa \neq 0)$

Kim (1989) solves for the homogeneous Neumann boundary-conditions 2.6b

$$
\frac{\partial G_{\mathrm{Kim}}}{\partial y}\left(y= \pm \frac{L_{y}}{2}, Y ; \kappa\right)=0 \stackrel{\text { B } 3}{\Longleftrightarrow} \frac{\partial q_{\mathrm{Kim}}}{\partial y}\left(y= \pm \frac{L_{y}}{2} ; \kappa\right)=0
$$

Straightforward calculations yield the functions $A_{1}(Y ; \kappa)$ and $A_{2}(Y ; \kappa)$ in $(\mathrm{B} 9)$ which satisfy (B10a), giving

$$
G_{\text {Kim }}(y, Y ; \kappa \neq 0)=-\frac{\cosh \left[\kappa\left(L_{y}-|y-Y|\right)\right]+\cosh [\kappa(y+Y)]}{2 \kappa \sinh \kappa L_{y}}
$$

This if-less expression, which highlights the symmetry (reciprocity) condition (B 4), is equivalent to the form in $\operatorname{Kim}(1989$, (8), p. 440), written here using dimensional variables

$$
G_{\text {Kim }}(y, Y ; \kappa \neq 0)= \begin{cases}-\frac{\cosh \left[\kappa\left(\frac{1}{2} L_{y}-Y\right)\right] \cosh \left[\kappa\left(\frac{1}{2} L_{y}+y\right)\right]}{\kappa \sinh \kappa L_{y}} & y \leq Y \\ -\frac{\cosh \left[\kappa\left(\frac{1}{2} L_{y}-y\right)\right] \cosh \left[\kappa\left(\frac{1}{2} L_{y}+Y\right)\right]}{\kappa \sinh \kappa L_{y}} & y \geq Y\end{cases}
$$

because using well-known identitiest (Harris \& Stocker 1998, p. 249)

$$
\begin{aligned}
2 \cosh \left[\kappa\left(\frac{1}{2} L_{y}-Y\right)\right] \cosh \left[\kappa\left(\frac{1}{2} L_{y}+y\right)\right]= & \cosh \left[\kappa\left(L_{y}-Y+y\right)\right]+\cosh [\kappa(-Y-y)] \\
= & \cosh \left[\kappa\left(L_{y}-|y-Y|\right)\right]+\cosh [\kappa(y+Y)] \\
& \forall y \leq Y \\
2 \cosh \left[\kappa\left(\frac{1}{2} L_{y}-y\right)\right] \cosh \left[\kappa\left(\frac{1}{2} L_{y}+Y\right)\right]= & \cosh \left[\kappa\left(L_{y}-y+Y\right)\right]+\cosh [\kappa(-y-Y)] \\
= & \cosh \left[\kappa\left(L_{y}-|y-Y|\right)\right]+\cosh [\kappa(y+Y)] \\
& \forall y \geq Y
\end{aligned}
$$

$\dagger \cosh (a+b)=\cosh a \cosh b+\sinh a \sinh b \quad \cosh (a-b)=\cosh a \cosh b-\sinh a \sinh b$ 


\section{B.2.2. Accommodating the boundary-conditions $(\kappa \neq 0)$}

To take into account normal gradients $d_{y} q\left(y= \pm \frac{1}{2} L_{y} ; \kappa \neq 0\right)=B_{ \pm}(\kappa \neq 0) \neq 0$ at the channel walls, corresponding to the Stokes pressure $2.3,2.6)$, Chang et al. $(1999)$ superpose a boundary-condition $q_{\mathrm{BC}}$ term to the solution of $\operatorname{Kim}(1989)$

$$
\begin{gathered}
q\left(y ; \kappa \neq 0, B_{ \pm}\right)=\int_{-\frac{L_{y}}{2}}^{+\frac{L_{y}}{2}} G_{\mathrm{Kim}}(y, Y ; \kappa \neq 0) Q(Y ; \kappa \neq 0) d Y+q_{\mathrm{BC}}\left(y ; \kappa \neq 0, B_{ \pm}\right) \\
\frac{d^{2} q\left(y ; \kappa \neq 0, B_{ \pm}\right)}{d y^{2}}-\kappa^{2} q\left(y ; \kappa \neq 0, B_{ \pm}\right)=Q(y ; \kappa \neq 0) \quad y \in\left(-\frac{1}{2} L_{y},+\frac{1}{2} L_{y}\right) \\
\frac{d^{2} q_{\mathrm{BC}}\left(y ; \kappa \neq 0, B_{ \pm}\right)}{d y^{2}}-\kappa^{2} q_{\mathrm{BC}}\left(y ; \kappa \neq 0, B_{ \pm}\right)=0 \quad y \in\left(-\frac{1}{2} L_{y},+\frac{1}{2} L_{y}\right) \\
\frac{d q}{d y}\left(y= \pm \frac{1}{2} L_{y} ; \kappa \neq 0, B_{ \pm}\right) \frac{\operatorname{B} 10 a}{-} \frac{d q_{\mathrm{BC}}}{d y}\left(y= \pm \frac{1}{2} L_{y} ; \kappa \neq 0, B_{ \pm}\right)=B_{ \pm}(\kappa \neq 0)
\end{gathered}
$$

By B3, B 10), the superposition (B11a) is the solution to $\mathrm{B} 11 b)$, with boundaryconditions $(\mathrm{B} 11 d)$. The solution of $(\mathrm{B} 11 \mathrm{c})$ with boundary-conditions $(\mathrm{B} 11 d)$ is readily obtained by direct integration (Chang et al. 1999, Foysi et al. 2004), and reads

$$
q_{\mathrm{BC}}\left(y ; \kappa \neq 0, B_{ \pm}\right)=\frac{B_{+} \cosh \left[\kappa\left(\frac{1}{2} L_{y}+y\right)\right]-B_{-} \cosh \left[\kappa\left(\frac{1}{2} L_{y}-y\right)\right]}{\kappa \sinh \kappa L_{y}}
$$

\section{B.2.3. Freespace Green's function $G_{\mathfrak{V}}(y, Y ; \kappa \neq 0)$}

The freespace Green's function is directly obtained from the general solution $\mathrm{B} 9 \mathrm{~b}$ requiring that the solution should tend to 0 as $y \rightarrow \pm \infty$, for any finite distribution of sources $Q(y ; \kappa \neq 0)$ with compact support, which is equivalent to

$$
\lim _{y \rightarrow \pm \infty} q_{\mathfrak{V}}(y ; \kappa \neq 0)=0 \stackrel{\mathrm{B} 3)}{\Longrightarrow} \lim _{y \rightarrow \pm \infty} G_{\mathfrak{V}}(y, Y ; \kappa \neq 0)=0 \quad \forall Y \in \mathbb{R} \backslash\{y\}
$$

Using (B 13) to determine the functions $A_{1}(Y ; \kappa)$ and $A_{2}(Y ; \kappa)$ in $(\mathrm{B} 9)$ readily gives

$$
G_{\mathfrak{V}}(y, Y ; \kappa \neq 0)=-\frac{\mathrm{e}^{-\kappa|y-Y|}}{2 \kappa}
$$

\section{B.2.4. Upper/lower wall Green's functions $G_{ \pm}(y, Y ; \kappa \neq 0)$}

These Green's functions correspond to the problems where the domain of interest is $\left(-\infty, \frac{1}{2} L_{y}\right)$ (isolated upper-wall influence; $\left.G_{+}\right)$or $\left(-\frac{1}{2} L_{y},+\infty\right)$ (isolated lower-wall influence; $\left.G_{-}\right)$, ie they are halfspace problems $\left( \pm y \in\left(-\infty, \frac{1}{2} L_{y}\right)\right)$. For each of these problems only one of the walls is present. The associated boundary-conditions are 0gradient at the wall of the problem $\left(y= \pm \frac{1}{2} L_{y}\right)$, and bounded influence at the infinity of the problem $(y \rightarrow \mp \infty)$, ie in terms of Green's functions

$$
\begin{array}{r}
\left.G_{+}(y, Y ; \kappa \neq 0) \text { satisfies } \mathrm{B} 2 \Longrightarrow \mathrm{B} 3, \mathrm{~B} 7\right) \\
\frac{\partial G_{+}}{\partial y}\left(y=+\frac{1}{2} L_{y}, Y ; \kappa \neq 0\right)=0 \\
\lim _{y \rightarrow-\infty} G_{+}(y, Y ; \kappa \neq 0)=0 \quad \forall Y \in \mathbb{R} \backslash\{y\}
\end{array}
$$


to study the virtual influence of the upper wall, and

$$
\begin{array}{r}
G_{-}(y, Y ; \kappa \neq 0) \text { satisfies } \mathrm{B} 2 \Longrightarrow \mathrm{B} 3, \mathrm{~B} 7 \\
\frac{\partial G_{-}}{\partial y}\left(y=-\frac{1}{2} L_{y}, Y ; \kappa \neq 0\right)=0 \\
\lim _{y \rightarrow+\infty} G_{-}(y, Y ; \kappa \neq 0)=0 \quad \forall Y \in \mathbb{R} \backslash\{y\}
\end{array}
$$

to study the virtual influence of the lower wall. By (B 3), boundary-conditions B $15 b$. B 16b imply 0-gradient at the wall of the problem (upper for $G_{+}$and lower for $G_{-}$). Boundary-conditions $(\overline{\mathrm{B} 15 c}, \mathrm{~B} 16 c)$ ensure boundedness at infinity for the halfspace corresponding to each problem. Straightforward computation of the functions $A_{1}(Y ; \kappa)$ and $A_{2}(Y ; \kappa)$ in $\left.\mathrm{B} 9\right)$ yields

$$
\begin{array}{ll}
G_{-}(y, Y ; \kappa \neq 0)=-\frac{\mathrm{e}^{-\kappa|y-Y|}}{2 \kappa}-\frac{\mathrm{e}^{-\kappa\left(L_{y}+y+Y\right)}}{2 \kappa} & y, Y \in\left[-\frac{1}{2} L_{y},+\infty\right) \\
G_{+}(y, Y ; \kappa \neq 0)=-\frac{\mathrm{e}^{-\kappa|y-Y|}}{2 \kappa}-\frac{\mathrm{e}^{-\kappa\left(L_{y}-y-Y\right)}}{2 \kappa} & y, Y \in\left(-\infty,+\frac{1}{2} L_{y}\right]
\end{array}
$$

which by $\mathrm{B} 14$ reads

$$
\begin{array}{ll}
G_{-}(y, Y ; \kappa \neq 0)=G_{\mathfrak{V}}(y, Y ; \kappa \neq 0)-\frac{\mathrm{e}^{-\kappa\left(L_{y}+y+Y\right)}}{2 \kappa} & y, Y \in\left[-\frac{1}{2} L_{y},+\infty\right) \\
G_{+}(y, Y ; \kappa \neq 0)=G_{\mathfrak{V}}(y, Y ; \kappa \neq 0)-\frac{\mathrm{e}^{-\kappa\left(L_{y}-y-Y\right)}}{2 \kappa} & y, Y \in\left(-\infty,+\frac{1}{2} L_{y}\right]
\end{array}
$$

$i e$ the presence of a single wall in any of the 2 halfspace problems induces an additive correction to the freespace Green's function, the wall-echo $G_{w_{ \pm}}(y, Y ; \kappa \neq 0)$

$$
G_{w_{ \pm}}(y, Y ; \kappa \neq 0):=G_{ \pm}(y, Y ; \kappa \neq 0)-G_{\mathfrak{V}}(y, Y ; \kappa \neq 0)
$$

\section{B.2.5. Accommodating the boundary-conditions for the halfspace problems $(\kappa \neq 0)$}

To take into account normal gradients $d_{y} q\left(y= \pm \frac{1}{2} L_{y} ; \kappa \neq 0\right)=B_{ \pm}(\kappa \neq 0) \neq 0$ at the wall for each of the halfspace problems of $\$ \mathrm{~B} .2 .4$ we may proceed exactly as in $\S \overline{\mathrm{B} .2 .2}$, by superposing a boundary-condition $q_{\mathrm{BC}_{ \pm}}$term to the corresponding solution (B 16) with 0-gradient condition, viz

$$
\begin{array}{r}
q\left(y ; \kappa \neq 0, B_{-}\right)=\int_{-\frac{L_{y}}{2}}^{+\frac{L_{y}}{2}} G_{-}(y, Y ; \kappa \neq 0) Q(Y ; \kappa \neq 0) d Y+q_{\mathrm{BC}_{-}}\left(y ; \kappa \neq 0, B_{-}\right) \\
\frac{d^{2} q\left(y ; \kappa \neq 0, B_{-}\right)}{d y^{2}}-\kappa^{2} q\left(y ; \kappa \neq 0, B_{-}\right)=Q(y ; \kappa \neq 0) \quad y \in\left(-\frac{1}{2} L_{y},+\infty\right) \\
\frac{d^{2} q_{\mathrm{BC}-}\left(y ; \kappa \neq 0, B_{-}\right)}{d y^{2}}-\kappa^{2} q_{\mathrm{BC}_{-}}\left(y ; \kappa, B_{-}\right)=0 \quad y \in\left(-\frac{1}{2} L_{y},+\infty\right) \\
\frac{d q}{d y}\left(y=-\frac{1}{2} L_{y} ; \kappa \neq 0, B_{-}\right) \frac{\mathrm{B} 16 b}{-} \frac{d q_{\mathrm{BC}_{-}}}{d y}\left(y=-\frac{1}{2} L_{y} ; \kappa \neq 0, B_{-}\right)=B_{-}(\kappa \neq 0) \\
\lim _{y \rightarrow+\infty} q_{\mathrm{BC}_{-}}\left(y ; \kappa \neq 0, B_{-}\right)=0
\end{array}
$$


to study the virtual influence of the lower wall boundary-condition on the solution, and

$$
\begin{array}{r}
q\left(y ; \kappa \neq 0, B_{+}\right)=\int_{-\frac{L_{y}}{2}}^{+\frac{L_{y}}{2}} G_{+}(y, Y ; \kappa \neq 0) Q(Y ; \kappa \neq 0) d Y+q_{\mathrm{BC}_{+}}\left(y ; \kappa \neq 0, B_{+}\right) \\
\frac{d^{2} q\left(y ; \kappa \neq 0, B_{+}\right)}{d y^{2}}-\kappa^{2} q\left(y ; \kappa \neq 0, B_{+}\right)=Q(y ; \kappa \neq 0) \quad y \in\left(-\infty,+\frac{1}{2} L_{y}\right) \\
\frac{d^{2} q_{\mathrm{BC}_{+}}\left(y ; \kappa \neq 0, B_{+}\right)}{d y^{2}}-\kappa^{2} q_{\mathrm{BC}_{+}}\left(y ; \kappa \neq 0, B_{+}\right)=0 \quad y \in\left(-\infty,+\frac{1}{2} L_{y}\right) \\
\frac{d q}{d y}\left(y=+\frac{1}{2} L_{y} ; \kappa \neq 0, B_{+}\right) \frac{\text { B } 15 b}{-} \frac{d q_{\mathrm{BC}_{+}}}{d y}\left(y=+\frac{1}{2} L_{y} ; \kappa \neq 0, B_{+}\right)=B_{+}(\kappa \neq 0) \\
\lim _{y \rightarrow-\infty} q_{\mathrm{BC}_{+}}\left(y ; \kappa \neq 0, B_{+}\right)=0
\end{array}
$$

to study the virtual influence of the upper wall boundary-condition on the solution. By (B 16), the superposition $(\mathrm{B} 20 a)$ is the solution to $(\mathrm{B} 20 b)$, with boundary-conditions B 20d. B 20e . By (B 15), the superposition (B21a) is the solution to B 21b), with boundary-conditions $(\mathrm{B} 21 \mathrm{~d}, \mathrm{~B} 21 \mathrm{e})$. Straightforward computation yields

$$
\begin{aligned}
& q_{\mathrm{BC}_{ \pm}}\left(y ; \kappa \neq 0, B_{ \pm}\right)= \pm \frac{B_{ \pm}}{\kappa} \mathrm{e}^{-\kappa\left(\frac{1}{2} L_{y} \mp y\right)} \\
& \text { B.3. } \kappa=0 \text { (xz-constant component) }
\end{aligned}
$$

When $\kappa=0$ (B 1) becomes a Poisson equation (Katz \& Plotkin 1991)

$$
\frac{d^{2} q(y ; \kappa=0)}{d y^{2}}=Q(y ; \kappa=0) \quad y \in\left(-\frac{1}{2} L_{y}, \frac{1}{2} L_{y}\right)
$$

The limits as $\kappa \rightarrow 0$ of the solutions obtained in $\$ \mathrm{~B} .2$ for $\kappa \neq 0$ are all singular, as can be easily verified by straightforward computation, contrary to the case of the same problem with Dirichlet boundary-conditions (Zauderer 2006). This is related to the existence of solutions of (B 23) with Neumann boundary-conditions at $y= \pm \frac{1}{2} L_{y}$, because integrating B 23) yields

$$
\frac{d q}{d y}\left(y=+\frac{1}{2} L_{y} ; \kappa=0\right)-\frac{d q}{d y}\left(y=-\frac{1}{2} L_{y} ; \kappa=0\right)=\int_{-\frac{1}{2} L_{y}}^{+\frac{1}{2} L_{y}} Q(Y ; \kappa=0) d Y
$$

implying that B 23) with gradient boundary-conditions $q^{\prime}\left(y= \pm \frac{1}{2} L_{y} ; \kappa=0\right)=B_{ \pm}(\kappa=$ 0 ) can only be solved iff the compatibility condition (B 24) is satisfied by the integral of the source-term and the boundary-conditions.

In the particular case of homogeneous Neumann boundary-conditions $B_{ \pm}(\kappa=0)=0$, which is the most important since $B_{ \pm}(\kappa=0) \neq 0$ is only concerned with Stokes pressure 2.3 2.6), the homogeneous equivalent of equation (B 23), $q^{\prime \prime}(y ; \kappa=0)=0$, admits any constant function as solution, ie any constant function is an eigenfunction (Ince 1926, Courant \& Hilbert 1953 Myint-U \& Debnath 2007) of the homogeneous equivalent of (B 23), $q^{\prime \prime}(y ; \kappa=0)=0$, with boundary-conditions $q^{\prime}\left(y= \pm \frac{1}{2} L_{y} ; \kappa=0\right)=0$, and $\kappa=0$ is a characteristic number of the homogeneous equivalent of $(\mathrm{B} 1), q^{\prime \prime}(y ; \kappa)-\kappa^{2} q(y ; \kappa)=0$ with boundary-conditions $q^{\prime}\left(y= \pm \frac{1}{2} L_{y} ; \kappa\right)=0$. Therefore a solution only exists provided the corresponding compatibility relation (Ince 1926: Courant \& Hilbert 1953) holds, ie the integral in (B 24) is equal to 0 . This is analogous to the 3-D compatibility relation for the Poisson equation with Neumann boundary-conditions (Ockendon et al. 2003 , Zauderer 2006). 
B.3.1. $G_{\operatorname{Kim}}(y, Y ; \kappa=0)$

In this case 2.3b 2.6b 0-gradient boundary-conditions apply on both walls ( $y=$ $\left.\pm \frac{1}{2} L_{y}\right)$. Assuming that the compatibility relation

$$
\int_{-\frac{1}{2} L_{y}}^{+\frac{1}{2} L_{y}} Q(Y ; \kappa=0) d Y=0
$$

holds, there are 2 different ways for constructing a modified Green's function (Courant \& Hilbert 1953, Zauderer 2006) for solving (B 23) with homogeneous Neumann boundaryconditions. The first method described in Courant \& Hilbert (1953) makes explicit use of the eigensolution of $(\mathrm{B} 23)$ in a modified definition of the problem $(\mathrm{B} 2)$ for calculating the modified Green's function which satisfies the boundary-conditions, but has also to modify (B 3). The second method (Zauderer 2006) which leads to the Green's function used by Kim (1989) uses the standard definition of the Green's function $(\mathrm{B} 2)$ along with the standard integral solution (B 3). In this latter case however the boundary-conditions are not explicitly included in the solution but are enforced by the compatibility relation B 25.

Hence, assuming that the compatibility relation $\mathrm{B} 25 \mathrm{~h}$ holds, so that $\mathrm{B} 10 \mathrm{a})$ for $\kappa=0$ may be satisfied, the Green's function corresponding to (B 23) satisfies

$$
\begin{array}{r}
\frac{\partial^{2} G_{\text {Kim }}(y, Y ; \kappa=0)}{\partial y^{2}}=\delta(y-Y) \\
\lim _{\epsilon \rightarrow 0^{+}}\left[\frac{\partial G_{\text {Kim }}}{\partial y}(Y+\epsilon, Y ; \kappa=0)-\frac{\partial G_{\text {Kim }}}{\partial y}(Y-\epsilon, Y ; \kappa=0)\right]=1 \\
G_{\text {Kim }}(y, Y ; \kappa=0) \in C^{0}\left(-\frac{1}{2} L_{y},+\frac{1}{2} L_{y}\right) \\
G_{\text {Kim }}(y, Y ; \kappa) \in C^{1}\left(-\frac{1}{2} L_{y},+\frac{1}{2} L_{y}\right) \backslash\{Y\}
\end{array}
$$

It is therefore made up by 2 straight lines joined together at $Y$, since by $(\mathrm{B} 26 a) \partial_{y y}^{2} G(y, Y ; \kappa=$ $0)=0 \forall y \neq Y$. If we further admit that $G_{\text {Kim }}(y, Y ; \kappa=0)=G_{\text {Kim }}(Y, y ; \kappa=0)(\mathrm{B} 4)$, as expected because of the self-adjointedness of the the operator $\left[d_{y y}^{2}(\cdot)\right] \sqrt{\text { Ince } 1926}$ Courant \& Hilbert 1953), we must have $\left(\operatorname{Katz} \&\right.$ Plotkin 1991) $G_{\operatorname{Kim}}(y, Y ; \kappa=0)=$ $G_{\text {Kim }}(|y-Y| ; \kappa=0)$ so that

$$
G_{\text {Kim }}(y, Y ; \kappa=0)=\frac{|y-Y|}{2}+c
$$

The factor $\frac{1}{2}$ is required to satisfy $(\mathrm{B} 26 b)$, and $c \in \mathbb{R}$ is a constant. The value of $c$ has no influence whatsoever on the solution (B 3 because of the compatibility condition (B 25) The simplest choice $c=0$ corresponding to the reasonable situation $G_{\operatorname{Kim}}(y, y ; \kappa=0)=0$ is made

$$
\begin{gathered}
G_{\operatorname{Kim}}(y, Y ; \kappa=0)=\frac{|y-Y|}{2} \\
\dagger \int_{-\frac{1}{2} L_{y}}^{+\frac{1}{2} L_{y}}\left(\frac{|y-Y|}{2}+c\right) Q(Y ; \kappa=0) d Y=\int_{-\frac{1}{2} L_{y}}^{+\frac{1}{2} L_{y}} \frac{|y-Y|}{2} Q(Y ; \kappa=0) d Y+c \int_{-\frac{1}{2} L_{y}}^{+\frac{1}{2} L_{y}} Q(Y ; \kappa=0) d Y \\
\stackrel{\text { B } 255}{=} \int_{-\frac{1}{2} L_{y}}^{+\frac{1}{2} L_{y}} \frac{|y-Y|}{2} Q(Y ; \kappa=0) d Y
\end{gathered}
$$


and we have

$$
\begin{gathered}
\frac{d q_{\mathrm{Kim}}}{d y}\left(y= \pm \frac{1}{2} L_{y} ; \kappa=0\right) \stackrel{\mathrm{B} 33}{=} \int_{-\frac{1}{2} L_{y}}^{+\frac{1}{2} L_{y}} \frac{\partial G_{\mathrm{Kim}}}{\partial y}(y, Y ; \kappa=0) Q(Y ; \kappa=0) d Y \\
\stackrel{\mathrm{B} 28}{-} \pm \frac{1}{2} \int_{-\frac{1}{2} L_{y}}^{+\frac{1}{2} L_{y}} Q(Y ; \kappa=0) d Y \stackrel{\mathrm{B} 25}{=} 0
\end{gathered}
$$

Notice that in this case it is the compatibility relation (B 25), and not the Green's function alone, which is responsible for satisfying the boundary-conditions.

B.3.2. Accommodating the boundary-conditions $(\kappa=0)$

Finally, the solution of the boundary-conditions problem (required for $p_{(\tau)}^{\prime}$ ) can be obtained directly by integrating

$$
\begin{array}{r}
\frac{d^{2} q_{\mathrm{BC}}\left(y ; \kappa=0, B_{ \pm}\right)}{d y^{2}}=0 \quad y \in\left(-\frac{1}{2} L_{y},+\frac{1}{2} L_{y}\right) \\
\frac{d q_{\mathrm{BC}}}{d y}\left(y= \pm \frac{1}{2} L_{y} ; \kappa=0, B_{ \pm}\right)=B_{ \pm}(\kappa=0)
\end{array}
$$

The solution exists iff

$$
B_{-}(\kappa=0)=B_{+}(\kappa=0)
$$

and is obviously a straight line, defined up to an additive constant

$$
q_{\mathrm{BC}}\left(y ; \kappa=0, B_{ \pm}\right)=\frac{1}{2} B_{-} y+\frac{1}{2} B_{+} y+q_{\mathrm{BC}_{0}}
$$

where by $\mathrm{B} 32 q_{\mathrm{BC}}(y=0 ; \kappa=0)=q_{\mathrm{BC}_{0}}$ is the solution at centerline.

B.3.3. Freespace Green's function $G_{\mathfrak{V}}(y, Y ; \kappa=0)$

The solution for the freespace Green's function $G_{\mathfrak{V}}(y, Y ; \kappa=0)$ is obtained exactly in the same way as for $G_{\operatorname{Kim}}(y, Y ; \kappa=0)$ (B.3.1), yielding by (B 28)

$$
G_{\mathfrak{V}}(y, Y ; \kappa=0)=G_{\text {Kim }}(y, Y ; \kappa=0)=\frac{|y-Y|}{2}
$$

Again, the sources $Q(y ; \kappa=0)$ must satisfy the compatibility condition $\mathrm{B} 25$ to ensure boundedness as $|y| \rightarrow \infty$

$$
\begin{aligned}
& \lim _{|y| \rightarrow \infty} \int_{-\frac{1}{2} L_{y}}^{+\frac{1}{2} L_{y}} G_{\mathfrak{V}}(y, Y \kappa=0) Q(Y ; \kappa=0) d Y \text { B }- \\
& \lim _{|y| \rightarrow \infty}\left\{\left(\int_{-\frac{1}{2} L_{y}}^{+\frac{1}{2} L_{y}} Q(Y ; \kappa=0) d Y\right) \frac{|y|}{2}\right\} \stackrel{\mathrm{B} 25 \mid}{-} 0 \quad \forall Y \in \mathbb{R} \backslash\{y\}
\end{aligned}
$$

which is 0 iff B 25) holds.

B.3.4. Upper/lower wall Green's functions $G_{ \pm}(y, Y ; \kappa=0)$

The solution for $G_{ \pm}(y, Y ; \kappa=0)$ is again obtained in exactly the same way as for $G_{\text {Kim }}(y, Y ; \kappa=0)($ B.3.1), yielding by B 33

$$
G_{ \pm}(y, Y ; \kappa=0)=G_{\mathfrak{V}}(y, Y ; \kappa=0)=G_{\operatorname{Kim}}(y, Y ; \kappa=0)=\frac{|y-Y|}{2}
$$

The boundary-conditions for the gradient at the wall $\left(y=\mp \frac{1}{2} L_{y}\right)$, and for boundedness at $\pm \infty$, are again satisfied by relations analogous to B 29 . B 34, iff the compatibility relation $\mathrm{B} 25$ holds. 
B.3.5. Accommodating the boundary-conditions for the halfspace problems $(\kappa=0)$

Finally, the solution of the boundary-conditions (required for $p_{(\tau)}^{\prime}$ ), in the case of the halfspace problems, can be obtained directly by integrating

$$
\begin{array}{r}
\frac{d^{2} q_{\mathrm{BC}_{ \pm}}\left(y ; \kappa=0, B_{ \pm}\right)}{d y^{2}}=0 \quad \mp y \in\left(-\frac{1}{2} L_{y},+\infty\right) \\
\frac{d q_{\mathrm{BC}_{ \pm}}}{d y}\left(y= \pm \frac{1}{2} L_{y} ; \kappa=0, B_{ \pm}\right)=B_{ \pm}(\kappa=0)
\end{array}
$$

The solution is obviously a straight line of slope $B_{ \pm}(\kappa=0)$, and is bounded as $|y| \rightarrow \infty$ iff the compatibility condition

$$
B_{ \pm}(\kappa=0)=0 \Longrightarrow q_{\mathrm{BC}_{ \pm}}\left(y ; \kappa=0, B_{ \pm}\right)=0
$$

holds.

\section{B.4. Approximation error in the method of images}

One interesting application of the halfspace problems is that, by superposition, they give the solution obtained by the method of images (Manceau et al. 2001). Indeed the method of images corresponds to applying the freespace Green's function on the domain $y \in\left(-\frac{1}{2} L_{y},+\frac{1}{2} L_{y}\right)$, and on 2 ghost domains $y \in\left(-\frac{3}{2} L_{y},-\frac{1}{2} L_{y}\right) \cup\left(+\frac{1}{2} L_{y},+\frac{3}{2} L_{y}\right)$, with appropriately reflected source-terms (Fig. 4)

$$
\begin{array}{rlrl}
Q_{\mathrm{MWL}}(y ; \kappa) & =Q\left(-L_{y}-y ; \kappa\right) & y \in\left(-\frac{3}{2} L_{y},-\frac{1}{2} L_{y}\right) \\
Q_{\mathrm{MWL}}(y ; \kappa)=\quad Q(y ; \kappa) & y \in\left(-\frac{1}{2} L_{y},+\frac{1}{2} L_{y}\right) \\
Q_{\mathrm{MWL}}(y ; \kappa)=Q\left(+L_{y}-y ; \kappa\right) & y \in\left(+\frac{1}{2} L_{y},+\frac{3}{2} L_{y}\right)
\end{array}
$$

and the approximation to the solution obtained by the method of images used by Manceau et al. (2001) is

$$
q_{\mathrm{MWL}}(y ; \kappa):=\int_{-\frac{3}{2} L_{y}}^{+\frac{3}{2} L_{y}} G_{\mathfrak{V}}(y, Y ; \kappa) Q_{\mathrm{MWL}}(Y ; \kappa) d Y
$$

It is straightforward to construct a kernel $G_{\mathrm{MWL}}(y, Y ; \kappa)$ which applied on the sourceterms in the actual computational domain $y \in\left(-\frac{1}{2} L_{y},-\frac{1}{2} L_{y}\right)$ will return the method of

$\dagger$ Although Manceau et al. (2001) neglect $q_{\mathrm{BC}}\left(y ; \kappa, B_{ \pm}\right)$, this can be added to $q_{\mathrm{MwL}}(y ; \kappa)(\mathrm{B} 39$ to obtain the approximation of the complete solution (B 11a). 
images approximation (B 39), because, defining $Y_{ \pm}:= \pm L_{y}-Y$, we have

$$
\begin{aligned}
q_{\mathrm{MWL}}(y ; \kappa)= & \int_{-\frac{3}{2} L_{y}}^{+\frac{3}{2} L_{y}} G_{\mathfrak{V}}(y, Y ; \kappa) Q_{\mathrm{MWL}}(Y ; \kappa) d Y \\
& \int_{-\frac{3}{2} L_{y}}^{-\frac{1}{2} L_{y}} G_{\mathfrak{V}}(y, Y ; \kappa) Q\left(-L_{y}-Y ; \kappa\right) d Y \\
& +\int_{-\frac{1}{2} L_{y}}^{+\frac{1}{2} L_{y}} G_{\mathfrak{V}}(y, Y ; \kappa) Q(Y ; \kappa) d Y \\
& +\int_{+\frac{1}{2} L_{y}}^{+\frac{3}{2} L_{y}} G_{\mathfrak{V}}(y, Y ; \kappa) Q\left(+L_{y}-Y ; \kappa\right) d Y \\
= & -\int_{+\frac{1}{2} L_{y}}^{-\frac{1}{2} L_{y}} G_{\mathfrak{V}}\left(y,-L_{y}-Y_{-} ; \kappa\right) Q\left(Y_{-} ; \kappa\right) d Y_{-} \\
& +\int_{-\frac{1}{2} L_{y}}^{+\frac{1}{2} L_{y}} G_{\mathfrak{V}}(y, Y ; \kappa) Q(Y ; \kappa) d Y \\
& -\int_{+\frac{1}{2} L_{y}}^{-\frac{1}{2} L_{y}} G_{\mathfrak{V}}\left(y, L_{y}-Y_{+} ; \kappa\right) Q\left(Y_{+} ; \kappa\right) d Y_{+}
\end{aligned}
$$

yielding

$$
\begin{aligned}
q_{\mathrm{MWL}}(y ; \kappa) & =\int_{-\frac{1}{2} L_{y}}^{+\frac{1}{2} L_{y}} G_{\mathrm{MWL}}(y, Y ; \kappa) Q(Y ; \kappa) d Y \\
G_{\mathrm{MWL}}(y, Y ; \kappa) & :=G_{\mathfrak{V}}(y, Y ; \kappa)+G_{\mathfrak{V}}\left(y,-L_{y}-Y ; \kappa\right)+G_{\mathfrak{V}}\left(y, L_{y}-Y ; \kappa\right)
\end{aligned}
$$

and by $\mathrm{B} 14$

$$
G_{\mathrm{MWL}}(y, Y ; \kappa \neq 0) \frac{\mathrm{B} 14 \mid}{-}-\frac{\mathrm{e}^{-\kappa|y-Y|}}{2 \kappa}-\frac{\mathrm{e}^{-\kappa\left|L_{y}+y+Y\right|}}{2 \kappa}-\frac{\mathrm{e}^{-\kappa\left|L_{y}-y-Y\right|}}{2 \kappa}
$$

Notice that (B 41) gives $f$ the solution by integrating the sources in the actual domain $Y \in\left[-\frac{1}{2} L_{y},+\frac{1}{2} L_{y}\right]$ with an approximate Green's functions $G_{\mathrm{MWL}}(y, Y ; \kappa)(\mathrm{B} 41 b)$. This representation (B 41) readily yields the approximation error of the method of images, by comparison with the exact solution $(\mathrm{B} 10 b)$ obtained using $G_{\text {Kim }}(y, Y ; \kappa)$. If we consider the actual domain $y, Y \in\left[-\frac{1}{2} L_{y},+\frac{1}{2} L_{y}\right]$, we have

$$
\left.\begin{array}{l}
-\frac{1}{2} L_{y} \leq y \leq+\frac{1}{2} L_{y} \\
-\frac{1}{2} L_{y} \leq Y \leq+\frac{1}{2} L_{y}
\end{array}\right\} \Longrightarrow\left\{\begin{array}{l}
L_{y}+(y+Y) \geq 0 \\
L_{y}-(y+Y) \geq 0
\end{array}\right.
$$


so that

$$
\begin{aligned}
& G_{\mathrm{MWL}}(y, Y ; \kappa \neq 0) \stackrel{\mathrm{B} 41 c] \mathrm{B} 42}{-}-\frac{\mathrm{e}^{-\kappa|y-Y|}}{2 \kappa} \\
& -\frac{\mathrm{e}^{-\kappa\left(L_{y}+y+Y\right)}}{2 \kappa} \\
& -\frac{\mathrm{e}^{-\kappa\left(L_{y}-y-Y\right)}}{2 \kappa} \quad \forall y, Y \in\left[-\frac{1}{2} L_{y},+\frac{1}{2} L_{y}\right] \\
& \text { B 14] } G_{\mathfrak{V}}(y, Y ; \kappa \neq 0) \\
& +\underbrace{G_{-}(y, Y ; \kappa \neq 0)-G_{\mathfrak{V}}(y, Y ; \kappa \neq 0)}_{G_{w_{-}}(y, Y ; \kappa \neq 0)(\mathrm{B} 19)} \\
& +\underbrace{G_{+}(y, Y ; \kappa \neq 0)-G_{\mathfrak{V}}(y, Y ; \kappa \neq 0)}_{G_{w_{+}}(y, Y ; \kappa \neq 0)(\mathrm{B} 19)} \\
& \forall y, Y \in\left[-\frac{1}{2} L_{y},+\frac{1}{2} L_{y}\right]
\end{aligned}
$$

$i e$, the images problem (Manceau et al. 2001) is equivalent to adding to the freespace Green's function an independent wall-echo correction for each wall, the wall-echo correction for the upper (lower) wall being exactly the wall-echo of the halfspace problem with only the upper (lower) wall present $(\mathrm{B} .2 .4)$. Notice that by $\mathrm{B} 35) G_{w_{-}}(y, Y ; \kappa=0)=$ $G_{w_{+}}(y, Y ; \kappa=0)=0$ so that $(\mathrm{B} 42 c)$ holds $\forall \kappa \in \mathbb{R}_{\geq 0}$.

By $\mathrm{B} 10 b, \mathrm{~B} 42 b$ we may directly compute the error made when approximating the Green's function by the method of images

$$
\begin{array}{r}
\frac{1}{L_{y}}\left(G_{\mathrm{MWL}}(y, Y ; \kappa \neq 0)-G_{\mathrm{Kim}}(y, Y ; \kappa \neq 0)\right) \\
\frac{\mathrm{e}^{-\kappa L_{y}}}{2 \kappa L_{y} \sinh \kappa L_{y}}\left(\cosh \kappa|y-Y|+\mathrm{e}^{-\kappa L_{y}} \cosh \kappa(y+Y)\right) \\
\forall y, Y \in\left[-\frac{1}{2} L_{y},+\frac{1}{2} L_{y}\right]
\end{array}
$$

where $\mathrm{B} 43$ was made nondimensional by dividing the Green's functions by $L_{y}$. Obviously

$$
\left.y, Y \in\left[-\frac{1}{2} L_{y},+\frac{1}{2} L_{y}\right] \Longrightarrow \begin{array}{l}
0 \leq|y-Y| \leq L_{y} \\
0 \leq|y+Y| \leq L_{y}
\end{array}\right\} \Longrightarrow\left\{\begin{array}{l}
0 \leq \cosh \kappa|y-Y| \leq \cosh \kappa L_{y} \\
0 \leq \cosh \kappa|y+Y| \leq \cosh \kappa L_{y}
\end{array}\right.
$$

since $\cosh x=\cosh |x|$ is positive ans strictly increasing $\forall|x| \in \mathbb{R}_{\geq 0}$. Hence $\dagger$

$$
\begin{array}{r}
\frac{1}{L_{y}}\left(\max _{y, Y \in\left[-\frac{1}{2} L_{y},+\frac{1}{2} L_{y}\right]}\left|G_{\mathrm{MWL}}(y ; \kappa)-G_{\mathrm{Kim}}(y, Y ; \kappa)\right|\right) \leq \\
\underbrace{\frac{\mathrm{e}^{-\kappa L_{y}}\left(1+\mathrm{e}^{-\kappa L_{y}}\right) \cosh \kappa L_{y}}{2 \kappa L_{y} \sinh \kappa L_{y}}}_{E_{\mathrm{MWL}}\left(\kappa L_{y}\right)}
\end{array}
$$

showing that the upper bound of approximation error made by the method of images is a function of the nondimensional (outer scaling) wavenumber $\kappa L_{y}$ (Fig. 5).

$\dagger$ notice also that by B $43 G_{\mathrm{MWL}}(y, Y ; \kappa \neq 0)-G_{\mathrm{Kim}}(y, Y ; \kappa \neq 0) \geq 0 \forall y, Y \in\left[-\frac{1}{2} L_{y},+\frac{1}{2} L_{y}\right]$ 


\section{REFERENCES}

Del Álamo, J. C. \& JimÉnez, J. 2003 Spectra of the very large anisotropic scales in turbulent channels. Phys. Fluids 15 (6), L41-L44.

del Álamo, J. C., Jiménez, J., Zandonade, P. \& Moser, R. D. 2004 Scaling of the energy spectra of turbulent channels. J. Fluid Mech. 500, 135-144.

BAlAkumar, B. J. \& AdRiAn, R. J. 2007 Large- and very-large scale motions in channel and boundary-layer flows. Phil. Trans. Roy. Soc. London A 365, 665-681.

Bender, C. M. \& Orszag, S. A. 1978 Advanced Mathematical Methods for Scientists and Engineers, pp. 16-19 (Green's function for ODEs). New York [NY, USA]: Springer.

Bisplinghoff, R. L. \& Ashley, H. 1962 Principles of Aeroelasticity, p. 76. New York [Ny, USA]: Wiley.

Briggs, W. L. \& Henson, V. E. 1995 The DFT: An Owner's Manual for the Discrete Fourier Transform, pp. 144-150 (2-D DFT). Philadelphia [PA, USA]: SIAM.

Chang, III, P. A., Piomelli, U. \& Blake, W. K. 1999 Relations between wall-pressure and velocity-field sources. Phys. Fluids 11 (11), 3434-3448.

Cheng, H., Huang, J. \& Leiterman, T. J. 2006 An adaptive fast solver for the modified Helmholtz equation in 2-D. J. Comp. Phys. 211, 616-637.

Chou, P. Y. 1945 On velocity correlations and the solutions of the equations of turbulent fluctuations. Quart. Appl. Math. 3, 38-54.

Coleman, G. N., Kim, J. \& Moser, R. D. 1995 A numerical study of turbulent supersonic isothermal-wall channel flow. J. Fluid Mech. 305, 159-183.

Concos, G. M. 1964 The structure of the turbulent pressure field in boundary-layer flows. $J$. Fluid Mech. 18, 353-378.

Cormack, D. E. 1975 Topics in geophysical fluid dynamics: I. natural convection in shallow cavities, II. studies of a phenomenological turbulence model. PhD thesis, California Institute of Technology, Pasadena, California [CA, USA].

Courant, R. \& Hilbert, D. 1953 Methods of Mathematical Physics I, pp. 351-358 (Green's function for ODEs), 371-377 (examples of Green's function for ODEs). New York [NY, USA]: Interscience.

Craft, T. J. \& LAunder, B. 1996 A Reynolds-stress model designed for complex geometries. Int. J. Heat Fluid Flow 17, 245-254.

Daly, B. J. \& Harlow, F. H. 1970 Transport equations in turbulence. Phys. Fluids 13, $2634-2649$

Davidson, P. A. 2004 Turbulence. Oxford [GBR]: Oxford University Press.

DonAlDSOn, C. DUP. 1969 A computer study of an analytical model of boundary-layer transition. AIAA J. 7 (2), 271-278.

Dosio, A., Vilà-Guerau de Arellano, J., Holtslag, A. A. M. \& Builtjes, P. J. H. 2005 Relating Eulerian and Lagrangian statistics for the turbulent dispersion in the atmospheric convective boundary-layer. J. Atm. Sci. 62 (4), 1175-1191.

Durbin, P. A. 1993 A Reynolds-stress model for near-wall turbulence. J. Fluid Mech. 249, 465-498.

Durbin, P. A. 1995 Separated flow computation with the $\mathrm{k}-\varepsilon-v^{2}$ model. AIAA J. 33 (4), 659-664.

Durran, D. R. 1989 Improving the anelastic approximation. J. Atm. Sci. 46 (11), 1453-1461.

FAVre, A. 1965a Equations des gaz turbulents compressibles - I - formes générales. J. Méc. 4, 361-390.

FAvre, A. 1965b Equations des gaz turbulents compressibles - II - méthode des vitesses moyennes; méthode des vitesses moyennes pondérées par la masse volumique. J. Méc. 4, 391-421.

Foysi, H., SARkAR, S. \& Friedrich, R. 2004 Compressibility effects and turbulence scalings in supersonic channel flow. J. Fluid Mech. 509, 207-216.

Fu, S. 1993 Modelling of the pressure-velocity correlation in turbulence diffusion. Comp. Fluids 22, 199-205.

GARRICK, I. E. 1957 Nonsteady wing charactreristics. In Aerodynamic Components of Aircraft at High Speeds (ed. A. F. Donovan \& H. R. Lawrence), High Speed Aerodynamics and Jet Propulsion, vol. VII, pp. 658-793. Princeton, [NJ, USA]: Princeton University Press. 
Gerolymos, G. A., Lo, C. \& VAllet, I. $2012 a$ Tensorial representations of Reynolds-stress pressure-strain redistribution. ASME J. Appl. Mech. 79 (4), 044506(1-10).

Gerolymos, G. A., Lo, C., Vallet, I. \& Younis, B. A. $2012 b$ Term-by-term analysis of near-wall second moment closures. AIAA J. 50 (12), 2848-2864.

Gerolymos, G. A., Sauret, E. \& Vallet, I. 2004 Contribution to the single-point-closure Reynolds-stress modelling of inhomogeneous flow. Theor. Comp. Fluid Dyn. 17 (5-6), 407431.

Gerolymos, G. A., Sénéchal, D. \& Vallet, I. 2009 Very-high-order weno schemes. J. Comp. Phys. 228, 8481-8524.

Gerolymos, G. A., SÉnéchal, D. \& Vallet, I. 2010 Performance of very-high-order upwind schemes for DNS of compressible wall-turbulence. Int. J. Num. Meth. Fluids 63, 769-810.

Gerolymos, G. A. \& VAllet, I. 2001 Wall-normal-free near-wall Reynolds-stress closure for 3-D compressible separated flows. AIAA J. 39 (10), 1833-1842.

Gerolymos, G. A. \& Vallet, I. 2009 aerodynamics (a library and software package for computational aerodynamics). http://sourceforge.net/projects/aerodynamics.

Gibson, M. M. \& LAunder, B. E. 1978 Ground effects on pressure fluctuations in the atmospheric boundary-layer. J. Fluid Mech. 86, 491-511.

Goody, M. 2004 Empirical spectral model of surface pressure fluctuations. AIAA J. 42 (9), $1788-1794$.

HambA, F. 1999 Effects of pressure fluctuations on turbulence growth in compressible homogeneous shear flow. Phys. Fluids 11 (6), 1623-1635.

HANJALIĆ, K. 1994 Advanced turbulence closure models: A view of current status and future prospects. Int. J. Heat Fluid Flow 15, 178-203.

Harris, J. W. \& Stocker, H. 1998 Handbook of Mathematics and Computational Science. New York [NY, USA]: Springer Verlag.

HiRT, C. W. 1969 Generalized turbulence transport equations. Int Sem. of Int. Center for Heat and Mass Transfer, Herceg Novi, Yugoslavia.

Hoyas, S. \& JimÉnez, J. 2006 Scaling of the velocity fluctuations in turbulent channels up to $R e_{\tau}=2003$. Phys. Fluids 18, 011702(1-4).

HoyAs, S. \& JimÉnez, J. 2008 Reynolds number effects on the Reynolds-stress budgets in turbulent channels. Phys. Fluids 20, 101511(1-8).

Hu, Z. W., Morfey, C. L. \& Sandham, N. D. 2002 Aeroacoustics of wall-bounded turbulent flow. AIAA J. 40 (3), 465-473.

Hu, Z. W., Morfey, C. L. \& Sandham, N. D. 2006 Wall pressure and shear stress spectra from direct simulations of channel flow. AIAA J. 44 (7), 1541-1549.

Hu, Z. W. \& SAndham, N. D. 2001 DNs databases for turbulent Couette and Poiseuille flow. Tech. Rep. AFM-01-04. AFM Research Group, School of Engineering Sciences, University of Southampton.

Huang, P. G., Coleman, G. N. \& Bradshaw, P. 1995 Compressible turbulent channel flows: DNS results and modelling. J. Fluid Mech. 305, 185-218.

Hunt, J. C. R. \& Graham, J. M. R. 1978 Free-stream turbulence near plane boundaries. J. Fluid Mech. 84, 209-235.

Hutchins, N. \& MARusic, I. 2007 Evidence of very long meandering features in the logarithmic region of turbulent boundary-layers. J. Fluid Mech. 579, 1-28.

INCE, I. L. 1926 Ordinary Differential Equations, 1st edn., pp. 123-126 (adjoint linear differential operator), 254-263 (Green's functions), 266-269 (compatibility conditions). New York [NY, USA]: Dover.

JAKiRlić, S. \& HANJAlić, K. 2002 A new approach to modelling near-wall turbulence energy and stress dissipation. J. Fluid Mech. 459, 139-166.

Jovanović, J. 2004 The Statistical Dynamics of Turbulence. Berlin [D]: Springer.

Katz, J. \& Plotkin, A. 1991 Low-Speed Aerodynamics: From Wing Theory to Panel Methods, pp. 52-57 (kernel solution of the Laplace equation). New York [NY, UsA]: McGraw-Hill.

KIM, J. 1989 On the structure of pressure fluctuations in simulated turbulent channel-flow. $J$. Fluid Mech. 205, 421-451.

Kim, J., Moin, P. \& Moser, R. 1987 Turbulence statistics in fully developed channel flow at low-Reynolds-number. J. Fluid Mech. 177, 133-166. 
LAunder, B. E. \& Li, S. P. 1994 On the elimination of wall-topography parameters from 2-moment closure. Phys. Fluids 6, 999-1006.

Launder, B. E., Reece, G. J. \& Rodi, W. 1975 Progress in the development of a Reynoldsstress turbulence closure. J. Fluid Mech. 68, 537-566.

Lumley, J. L. 1978 Computational modeling of turbulent flows. Adv. Appl. Mech. 18, 123-176.

Mahle, I., Foysi, H., SARKar, S. \& Friedrich, R. 2007 On the turbulence structure of inert and reacting compressible mixing layers. J. Fluid Mech. 593, 171-180.

Manceau, R. \& Hanjalić, K. 2002 Elliptic blending model: A new near-wall Reynolds-stress turbulence closure. Phys. Fluids 14 (2), 744-754.

Manceau, R., Wang, M. \& Laurence, D. 2001 Inhomogeneity and anisotropy effects on the redistribution term in Reynolds-averaged Navier-Stokes modelling. J. Fluid Mech. 438, $307-338$.

Mansour, N. N., Kim, J. \& Moin, P. 1988 Reynolds-stress and dissipation-rate budgets in a turbulent channel flow. J. Fluid Mech. 194, 15-44.

Marmanis, H. 1998 Analogy between the Navier-Stokes equations and Maxwell's equations: Application to turbulence. Phys. Fluids 10 (6), 1428-1437.

Miles, J. W. 1959 The Potential Theory of Unsteady Supersonic Flow, p. 2ff. Cambridge [GBR]: Cambridge University Press.

Monin, A. S. \& Yaglom, A. M. 1971 Statistical fluid mechanics: Mechanics of turbulence, , vol. 1. Cambridge [MA, USA]: MIT Press.

Moser, R. D., Kim, J. \& Mansour, N. N. 1999 Direct numerical simulation of turbulent channel flow up to $R e_{\tau}=590$. Phys. Fluids 11 (4), 943-945.

Myint-U, T. \& Debnath, L. 2007 Linear Partial Differential Equations for Scientists and Engineers, 4th edn., pp. 310-317 (Green's function). Boston [MA, USA]: Birkhäuser.

Naka, Y., Omori, T., OBi, S. \& Masuda, S. 2006 Simultaneous measurements of fluctuating velocity and pressure in a turbulent mixing layer. Int. J. Heat Fluid Flow 27, 737-746.

Ockendon, J., Howison, S., Lacey, A. \& Movchan, A. 2003 Applied Partial Differential Equations, revised edn., pp. 163-166 (well-posedness for Poisson equation), 169-174 (Green's function). Oxford [GBR]: Oxford University Press.

Pantano, C. \& Sarkar, S. 2002 A study of compressibility effects in the high-speed turbulent shear layer using direct simulation. J. Fluid Mech. 451, 329-371.

PAnton, R. L. \& Linebarger, J. H. 1974 Wall pressure spectra calculations for equilibrium boundary-layers. J. Fluid Mech. 65, 261-287.

Perot, B. 1999 Turbulence modeling using body-force potentials. Phys. Fluids 11 (9), 26452656.

Piquet, J. 1999 Turbulent Flows - Models and Physics. Berlin [D]: Springer Verlag.

Pope, S. B. 2000 Turbulent Flows. Cambridge [GBR]: Cambridge University Press.

RistorCelLi, J. R. 1997 A pseudosound constitutive relationship for the dilatational covariances in compressible turbulence. J. Fluid Mech. 347, 37-70.

Ristorcelli, J. R., Lumley, J. L. \& ABID, R. 1995 A rapid-pressure covariance representation consistent with the Taylor-Proudman theorem materially frame indifferent in the 2-D limit. J. Fluid Mech. 292, 111-152.

RottA, J. 1951a Statistische Theorie nichthomogener Turbulenz - 1. Mitteilung. Z. Phys. 129, $547-572$.

RotтA, J. $1951 b$ Statistische Theorie nichthomogener Turbulenz - 2. Mitteilung. Z. Phys. 131, $51-77$.

SAURET, E. \& VAllet, I. 2007 Near-wall turbulent pressure diffusion modelling and influence in 3-D secondary flows. ASME J. Fluids Eng. 129 (5), 634-642.

Schwarz, W. R. \& Bradshaw, P. 1994 Term-by-term tests of stress-transport turbulence models in a 3-D boundary-layer. Phys. Fluids A 6 (2), 986-998.

Shin, T. H. \& Lumley, J. L. 1993 Critical comparison of 2-order closures with direct numerical simulations of homogeneous turbulence. AIAA J. 31 (4), 663-670.

SHIR, C. C. 1973 A preliminary numerical study of atmospheric turbulent flows in the idealized planetary boundary-layer. J. Atm. Sci. 30, 1327-1339.

So, R. M. C., Gatski, T. B. \& Sommer, T. P. 1998 Morkovin hypothesis and the modeling of wall-bounded compressible turbulent flows. AIAA J. 36 (9), 1583-1592. 
Speziale, C. G., Sarkar, S. \& Gatski, T. B. 1991 Modelling the pressure-strain correlation of turbulence: An invariant dynamical systems approach. J. Fluid Mech. 227, 245-272.

SugA, K. 2004 Modeling the rapid part of the pressure-diffusion process in the Reynolds stress transport equation. ASME J. Fluids Eng. 126, 634-641.

Taulbee, D. \& VAnOsdol, J. 1991 Modeling turbulent compressible flows: The mass fluctuating velocity and squared density. AIAA Paper 1991-0524.

Tsuji, Y., Fransson, J. H. M., Alfredsson, P. H. \& Johansson, A. V. 2007 Pressure statistics and their scalings in high-Reynolds-number turbulent boundary-layers. J. Fluid Mech. 585, 1-40.

Tsuji, Y. \& Ishinara, T. 2003 Similarity scaling of pressure fluctuation in turbulence. Phys. Rev. E 68, 026309(1-5).

VALLET, I. 2007 Reynolds-stress modelling of 3-D secondary flows with emphasis on turbulent diffusion closure. ASME J. Appl. Mech. 74 (6), 1142-1156.

ZAuderer, E. 2006 Partial Differential Equations of Applied Mathematics, pp. 446-447 (Green's function for ODEs), 451-454 (modified Green's function). Hoboken [NJ, USA]: Wiley. 